\title{
Bose-Einstein Condensation in Dilute Atomic Gases
}

\author{
A. F. R. de Toledo Piza \\ Instituto de Física, Universidade de São Paulo, C.P. 66318, 05315-970 São Paulo, SP, Brasil
}

Received on 8 February, 2004

\begin{abstract}
The material presented here consists of lecture notes written for a five two-hour sections course given during the 2004 edition of the Brazilian School on Statistical Mechanics. The topics covered are 1. Bose-Einstein condensation of trapped ideal gases; 2. Effective two-body interactions and exact results in the Gross-Pitaevski limit; 3. Atomic structure effects, including "Feshbach resonances" and hybrid condensates; 4. Elementary excitations in the Bogoliubov approximation and beyond; and 5. Splitt traps, interference phenomena and condensate arrays.
\end{abstract}

\section{Prologue}

The five lectures (or Chapters) which follow are intended as a pedagogical, theoretically oriented introduction to the presently very active field involving the physics of BoseEinstein condensation in trapped atomic gases. The lectures have been prepared for the 2004 Brazilian School on Statistical Mechanics, having in mind an audience of graduate and advanced undergraduate students possibly, and perhaps even typically, not concentrating in the subject. Needless to say, this makes them quite distinct from a review article on the subject, chiefly as considerations of impedance matching with the intended audience plays the dominant role in the choice and development of topics. This particular School is perhaps a somewhat peculiar setting for these lectures, since the systems which will be considered are orderly enough to be quite well described, for several relevant purposes, even in terms of a single one-body wavefunction. This will be discussed in lecture 2, after the statistical mechanics of the condensation phenomenon for an ideal Bose gas is discussed in lecture 1. A fortunate circumstance in this connection is that the condensation of an ideal gas is today no longer just a simplifying idealization, but an experimentally studied (i.e., real) phenomenon (see section 1.2). This will be discussed in some detail in lecture 3, together with the just now fashionable theme of molecular hybridization of dilute Bose-Einstein condensates and Bose-Einstein condensation of diatomic molecules formed in very cold fermionic gases. Lecture 4 will carry us slightly beyond the basic "single one-body wavefunction" description of real condensates, and lecture 5 will introduce experimental results on the optical fracturing of condensates as well as some of the ideas and simplified models used in connection with such situations.

In preparing these lectures, I have tried my best to avoid being trapped in what has been called by the former brazilian minister Pedro Malan in a newspaper article published on page 2 of $O$ Estado de São Paulo in January 11, 2004 (or was this also a quote?) "some kind of error contract" between someone trying to transmit thoughts and ideas and his "receivers". This syndrome has been supposedly described by none other than Francis Bacon. It was brought to Malan's attention (thus finding its way to his article and finally to this Prologue) by a book by another economist, Eduardo Giannetti da Fonseca, who identifies Bacon's The advancement of learning, as the source. As I have not been able to locate the original quote in time, I do my best translating (re-translating?) the brazilian version of the quote to English, certainly not Bacon's: "He who transmits knowledge chooses to do it so as to enhance belief rather than the possibility of examination, and who receives knowledge seeks rather present satisfaction than the promises of investigation, and thus will rather not doubt than not fail; glory leads the author not to reveal his weaknesses, and laziness leads the disciple not to realize his strength" ${ }^{1}$. Curious as my sources for these ideas happened to be, the dangers to

\footnotetext{
${ }^{1}$ Scanning once again Book 1 of Bacon's The advancement of learning I finally realized that at least some heavy editing has been involved in these in fact rather loose quotes. The closest, or most relevant, passages I have been able to find in Bacon's original work are transcribed here as they appear in the Renascence Editions "imprint" available on line at the site http://darkwing.uoregon.edu/rrbear/adv1.htm, see especially paragraph 9 of section V:

"BOOK 1, IV.12. And as for the overmuch credit that hath been given unto authors in sciences, in making them dictators, that their words should stand, and not counsellors to give advice; the damage is infinite that sciences have received thereby, as the principal cause that hath kept them low at a stay without growth or advancement. For hence it hath come, that in arts mechanical the first deviser comes shortest, and time addeth and perfecteth; but in sciences the first author goeth farthest, and time leeseth and corrupteth. So we see, artillery, sailing, printing, and the like, were grossly managed at the first, and by time accommodated and refined: but contrariwise, the philosophies and sciences of Aristotle, Plato, Democritus, Hippocrates, Euclides, Archimedes, of most vigour at the first and by time degenerate and imbased; whereof the reason is no other, but that in the former many wits and industries have contributed in one; and in the latter many wits and industries have been spent about the wit of some one, whom many times they have rather depraved than illustrated. For as water will not ascend higher than the level of the first springhead from whence it descendeth, so knowledge derived from Aristotle, and exempted from liberty of examination, will not rise again higher than the knowledge of Aristotle. And therefore although the position be good, OPORTET DISCENTEM CREDERE, yet it must be coupled with this, OPORTO EDOCTUM JUDICARE; for disciples do owe unto masters only a temporary belief and a suspension of their own judgment until they be fully instructed, and not an absolute resignation or perpetual captivity: and therefore, to conclude this point, I will say no more, but so let great authors have their due, as time, which is the author of authors, be not deprived of his due, which is, further and further to discover truth. Thus have I gone over these three diseases of learning; besides the which there are some other rather peccant humours that formed diseases: which
} 
which they refer are serious enough to deserve careful consideration. I thus hope that the content of the lectures will be doubted rather than believed, and tried to encourage this by not concealing remaining obscurities.

A word should be said on why these lectures, or Chapters, have been written in what amounts to the lingua franca ${ }^{2}$ of today. The organizers of the School said they intended to publish written versions of the courses in a special issue of the former Revista Brasileira de Fsica, now known as Brazilian Journal of Physics. My reaction to this was that it tended to create a certain conflict, as the scope, tone and purpose of course notes differ a lot from that of material usually printed even in special issues of periodicals. The intention having been maintained, my option has been to stick to the scope, tone and purpose of course notes while borrowing the use of the lingua franca from the periodical literature. Which might, after all, also have some pedagogical effect.

\section{Condensation of ideal bosons in a trap}

\subsection{Grand-canonical quantum statistics}

\section{Prolegomena: state vectors vs. density operators.}

States of thermal equilibrium of many-particle quantum systems cannot be represented by state vectors on the account of the fact that they do not correspond to definite microscopically defined states, but rather correspond to an incoherent distribution over the various possible such states. In this context, incoherent means that any interference effects involving different states in the distribution are precluded. One needs therefore a suitably extended way of describing quantum states in order to be able to deal with thermal equilibrium states.

The required extension is provided by the notion of density operators. Consider first how this notion arises in the particular situation in which the state of the system can be described in terms of a state vector. If the microscopically defined state of a many particle quantum system is characterized by a normalized state vector $|\psi\rangle,\langle\psi \mid \psi\rangle=1$ (or, equivalently, by a wavefunction $\psi\left(r_{1}, \ldots, r_{N}\right) \equiv\left\langle r_{1}, \ldots, r_{N} \mid \psi\right\rangle$, where $\left|r_{1}, \ldots, r_{N}\right\rangle$ is a Dirac eigenket of the position operators for the various particles), then it is characterized as well by the density operator, defined in this case as the projection operator

$$
\rho \equiv|\psi\rangle\langle\psi|
$$

or, equivalently, by the (N-particle) density matrix

$$
\begin{aligned}
\rho\left(r_{1}, \ldots, r_{N} ; r_{1}^{\prime}, \ldots, r_{N}^{\prime}\right) & \equiv\left\langle r_{1}, \ldots, r_{N} \mid \psi\right\rangle\left\langle\psi \mid r_{1}^{\prime}, \ldots, r_{N}^{\prime}\right\rangle \equiv \\
& \equiv \psi\left(r_{1}, \ldots, r_{N}\right) \psi^{*}\left(r_{1}^{\prime}, \ldots, r_{N}^{\prime}\right)
\end{aligned}
$$

nevertheless are not so secret and intrinsic but that they fall under a popular observation and traducement, and therefore are not to be passed over. (...)

V.6. Another error hath proceeded from too great a reverence, and a kind of adoration of the mind and understanding of man; by means whereof men have withdrawn themselves too much from the contemplation of nature, and the observations of experience, and have tumbled up and down in their own reason and conceits. Upon these intellectualists, which are notwithstanding commonly taken for the most sublime and divine philosophers, Heraclitus gave a just censure, saying, MEN SOUGHT TRUTH IN THEIR OWN LITTLE WORLDS, AND NOT IN THE GREAT AND COMMON WORLD; for they disdain to spell, and so by degrees to read in the volume of God's works: and contrariwise by continual meditation and agitation of wit do urge and as it were invocate their own spirits to divine and give oracles unto them, whereby they are deservedly deluded. (...)

9. Another error is in the manner of the tradition and delivery of knowledge, which is for the most part magistral and peremptory, and not ingenuous and faithful; in a sort as may be soonest believed, and not easiliest examined. I: is true, that in compendious treatises for practice that form is not to be disallowed: but in the true handling of knowledge, men ought not to fall either on the one side into the vein of Velleius the Epicurean: NIL TAM METUENS, QUAM NE DUBITARE ALIQUA DE RE VIDERETUR; [13] nor on the other side into Socrates his ironical doubting of all things; but to propound things sincerely with more or less asseveration, as they stand in a man's own judgment proved more or less. (...)

11. But the greatest error of all the rest is the mistaking or misplacing of the last or farthest end of knowledge: for men have entered into a desire of learning and knowledge, sometimes upon a natural curiosity and inquisitive appetite; sometimes to entertain their minds with variety and delight; sometimes for ornament and reputation; and sometimes to enable them to victory of wit and contradiction; and most times for lucre and profession; and seldom sincerely to give a true account of their gift of reason, to the benefit and use of men: as if there were sought in knowledge a couch whereupon to rest a searching and restless spirit; or a tarrasse, for a wandering and variable mind to walk up and down with a fair prospect; or a tower of state, for a proud mind to raise itself upon; or a fort or commanding ground, for strife and contention; or a shop, for profit or sale; and not a rich storehouse, for the glory of the Creator and the relief of man's estate. Rut this is that which will indeed dignify and exalt knowledge, if contemplation and action may be more nearly and straitly conjoined and united together than they have been; a conjunction like unto that of the two highest planets, Saturn, the planet of rest and contemplation, and Jupiter, the planet of civil society and action: howbeit, I do not mean, when I speak of use and action, that end before-mentioned of the applying of knowledge to lucre and profession; for I am not ignorant how much that diverteth and interrupteth the prosecution and advancement of knowledge, like unto the golden ball thrown before Atalanta, which while she goeth aside and stoopeth to take up, the race is hindered;

${ }^{2}$ Lingua Franca; a composite language made up of Italian and the various languages of western Asia, used in the Levant by foreign traders and natives of that region. (Webster's New Twentieth Century Dictionary of the English Language, Unabridged. Rockville House Publishers, Inc., New York, 1964). 
The normalization of the state vector translates into the property that the density operator, or matrix, has unit trace, i.e.

$$
\begin{gathered}
\operatorname{Tr} \rho \equiv \int d^{3} r_{1} \ldots \int d^{3} r_{N} \psi\left(r_{1}, \ldots, r_{N}\right) \psi^{*}\left(r_{1}, \ldots, r_{N}\right) \\
=\sum_{j}\left\langle\phi_{j} \mid \psi\right\rangle\left\langle\psi \mid \phi_{j}\right\rangle=1
\end{gathered}
$$

where the vectors $\left\{\left|\phi_{j}\right\rangle\right\}$ constitute an arbitrary orthonormal base in state vector space. In this case the operator $\rho$ is clearly an idempotent (i.e., $\rho^{2}=\rho$ ) self-adjoint operator. Its only possible eigenvalues are therefore 1 and 0 . The eigenvector corresponding to the eigenvalue 1 is clearly the state vector $|\psi\rangle$ itself (this is therefore how the original state vector $|\psi\rangle$ can be retrieved, given the corresponding idempotent density operator $\rho$ ), while the eigenvalue 0 is highly degenerate, since any state-vector orthogonal to $|\psi\rangle$ is an eigenvector associated to this eigenvalue. Note moreover that the expectation value of some given observable $O$ in the state $|\psi\rangle$ can be obtained directly from the density operator as

$$
\begin{aligned}
\operatorname{Tr} O \rho & \equiv \sum_{j}\left\langle\phi_{j}|O \rho| \phi_{j}\right\rangle=\sum_{j}\left\langle\phi_{j}|O| \psi\right\rangle\left\langle\psi \mid \phi_{j}\right\rangle= \\
& =\sum_{j}\left\langle\psi \mid \phi_{j}\right\rangle\left\langle\phi_{j}|O| \psi\right\rangle=\langle\psi|O| \psi\rangle
\end{aligned}
$$

the last step following from the completeness of the orthonormal base $\left\{\left|\phi_{j}\right\rangle\right\}$. In a similar way one can also verify the important cyclic property of the trace

$$
\operatorname{Tr} O \rho=\operatorname{Tr} \rho O
$$

which is in fact more general, in that it does not depend on the particular form of $\rho$.

The required generalization of the way to characterize states of quantum systems in order to include the needed incoherent distributions of state vectors consists in replacing the idempotency condition $\rho^{2}=\rho$ by the weaker condition that $\rho$ is a non-negative self-adjoint operator with unit trace. The non-negativity condition means that all the eigenvalues are non-negative, i.e. they are positive or zero. These density operators can be conveniently written in terms of their eigenvalues and normalized eigenvectors $\left|R_{j}\right\rangle$ as

$$
\rho\left|R_{j}\right\rangle=p_{j}\left|R_{j}\right\rangle, \quad p_{j} \geq 0, \quad \longrightarrow \quad \rho=\sum_{j}\left|R_{j}\right\rangle p_{j}\left\langle R_{j}\right| .
$$

The unit trace condition is now expressed as $\sum_{j} p_{j}=1$. The idempotent density operators are clearly particular cases of this more general class, which include moreover positive linear combinations of many (possibly even an infinite number of) orthogonal projection operators. The unit trace condition (actually the condition that the trace is finite is of course sufficient) in fact restricts a great deal the spectrum of the more general density operators: by virtue of the
Hilbert-Schmidt theorem it is guaranteed to be a purely discrete spectrum; and when the number of non-zero eigenvalues is infinite, they can only have zero as an accumulation point.

Average values of observables in states described by these density operators are also calculated in terms of a trace, which gives now

$$
\operatorname{Tr} O \rho=\sum_{j} O\left|R_{j}\right\rangle p_{j}\left\langle R_{j}\right|=\sum_{j} p_{j}\left\langle R_{j}|O| R_{j}\right\rangle .
$$

This average appears thus as a weighted average of quantum expectation values in the state vectors $\left|R_{j}\right\rangle$, with weights $p_{j}$. A standard interpretation of this is that the density operator $\rho$ describes an ensemble of systems, in which the (classical) probability of finding a system in state $\left|R_{j}\right\rangle$ is $p_{j}$. A particularly relevant property of the state represented by the density operator $\rho$, which gives a measure of fragmentation of the trace into the array of classical probabilities is its entropy $\mathrm{S}$ (sometimes called the von Neumann entropy), defined as

$$
S=-k_{B} \operatorname{Tr}(\rho \ln \rho)=-k_{B} \sum_{j} p_{j} \ln p_{j}
$$

For $k_{B}>0$ this is a non-negative quantity which vanishes in the limit of an idempotent density operator. If the constant $k_{B}$ is taken as the Boltzmann constant, $S$ has units of the standard thermodynamic entropy.

A further step is still needed when one wishes to use grand-canonical methods, as it is often the case in the context of Bose-Einstein condensation. In this case the number of particles in the many-particle system under consideration is not fixed and must be seen as an observable. The way to accommodate this is to use the language of "second quantization" (see, e.g., ref. [1], Chapter 7). The state vectors now reside in a "Fock space", or occupation number space, in which a number operator can be defined. The basic objects representing states of thermal equilibrium of quantum many-particle systems are thus to be taken as positive selfadjoint operators of unit trace in Fock space.

2. Grand-canonical equilibrium density operator. We now consider specifically a system of many identical bosonic atoms characterized by a hamiltonian $H$. For the purposes of the formal developments to be undertaken at this point, this may include interactions between atoms, e.g. represented by a suitable two-body potential $v\left(r_{j}, r_{l}\right)$, in addition to an external, one-body confining potential representing the trap. The state of the system is described by a density operator $\rho$ in Fock space. In this space we have also a number operator $N$, and the hamiltonian is represented by an operator which commutes with $N$. This means that one could adopt a canonical formulation by restricting the treatment to the $N$-particle sector of the Fock space, which is closed under the action of the number-conserving hamiltonian $H$, but 
it will be convenient to allow for states in which neither the number of atoms nor the energy are sharply defined.

The problem we are set to solve is to determine the density operator which makes the entropy an extremum, with prescribed average values for the energy and number of atoms. The density operator is written in the form (1), so that the average values of the hamiltonian $H$ and of the number operator $N$ are given respectively by (see eq. (2))

$$
\operatorname{Tr} H \rho=\sum_{j} p_{j}\left\langle R_{j}|H| R_{j}\right\rangle
$$

and

$$
\operatorname{Tr} N \rho=\sum_{j} p_{j}\left\langle R_{j}|N| R_{j}\right\rangle
$$

The entropy is expressed as in eq. (3), provided the weights satisfy the condition $\sum_{j} p_{j}=1$ and the states $\left|R_{j}\right\rangle$ are normalized, $\left\langle R_{j} \mid R_{j}\right\rangle=1$. These subsidiary conditions can all be taken care of in terms of Lagrange multipliers $\beta, \alpha, \lambda$ and $\eta_{j}$, so that the variational condition for the thermal equilibrium state is

$$
\delta\left(-k_{B} \sum_{j} p_{j} \ln p_{j}-\beta \sum_{j} p_{j}\left\langle R_{j}|H| R_{j}\right\rangle-\alpha \sum_{j} p_{j}\left\langle R_{j}|N| R_{j}\right\rangle-\lambda \sum_{j} p_{j}+\sum_{j} \eta_{j}\left\langle R_{j} \mid R_{j}\right\rangle\right)=0 .
$$

Variation of $p_{j}$ gives

$$
k_{B} \ln p_{j}+k_{B}+\beta\left\langle R_{j}|H| R_{j}\right\rangle+\alpha\left\langle R_{j}|N| R_{j}\right\rangle+\lambda=0
$$

while variation of $\left\langle R_{j}\right|$ leads to

$$
\begin{aligned}
p_{j}(\beta H+\alpha N)\left|R_{j}\right\rangle & =\eta_{j}\left|R_{j}\right\rangle \quad \text { or } \\
(\beta H+\alpha N)\left|R_{j}\right\rangle & =\frac{\eta_{j}}{p_{j}}\left|R_{j}\right\rangle \equiv \epsilon_{j}\left|R_{j}\right\rangle,
\end{aligned}
$$

which shows that the states $\left|R_{j}\right\rangle$ are eigenstates of $\beta H+$ $\alpha N$, so that this operator and $\rho$ are simultaneously diagonal, with $\beta\left\langle R_{j}|H| R_{j}\right\rangle+\alpha\left\langle R_{j}|N| R_{j}\right\rangle=\epsilon_{j}$. Straightforward algebra now gives, from (1.4),

$$
p_{j}=e^{-1-\lambda / k_{B}} e^{-\epsilon_{j} / k_{B}}
$$

which, using the unit trace condition to evaluate the first exponential, leads to

$$
p_{j}=\frac{e^{-\epsilon_{j} / k_{B}}}{\sum_{j} e^{-\epsilon_{j} / k_{B}}} .
$$

The denominator of this expression can be written as $\operatorname{Tr}\left(e^{-(\beta H+\alpha N) / k_{B}}\right)$, so that the resulting form for the density operator is

$$
\rho=\frac{\sum_{j}\left|R_{j}\right\rangle e^{-\epsilon_{j} / k_{B}}\left\langle R_{j}\right|}{\operatorname{Tr}\left(e^{-(\beta H+\alpha N) / k_{B}}\right)}=\frac{e^{-(\beta H+\alpha N) / k_{B}}}{\operatorname{Tr}\left(e^{-(\beta H+\alpha N) / k_{B}}\right)} .
$$

The meaning of the Lagrange multipliers $\lambda, \beta$ and $\alpha$ in macroscopic terms can be found by comparing the statistical expression for the entropy which results from imposing the variational condition (1.4), namely

$$
S=k_{B}+\lambda+\beta \operatorname{Tr} H \rho+\alpha \operatorname{Tr} N \rho,
$$

with the corresponding thermodynamic expression

$$
S=-\frac{1}{T}(\Omega-U+\mu N)
$$

where $T$ is the temperature, $\Omega$ is the grand potential, $\mu$ is the chemical potential, $N$ is the number of particles and $U$ is the internal energy. This allows one to make the identifications

$k_{B}+\lambda=-\frac{\Omega}{T} ; \quad \beta=\frac{1}{T} \quad$ and $\quad \alpha=-\frac{\mu}{T}$

The grand-canonical equilibrium density operator at temperature $T$ becomes accordingly

$$
\rho=\frac{e^{-\frac{1}{k_{B} T}(H-\mu N)}}{\operatorname{Tr}\left(e^{-\frac{1}{k_{B} T}(H-\mu N)}\right)} .
$$

The chemical potential $\mu$ can still be seen as a Lagrange multiplier to be determined by the subsidiary condition on the average total number of particles, $\operatorname{Tr} N \rho=\langle N\rangle$.

Some general comments are in order at this point. First, note that the Bose-Einstein statistics (in the case one is dealing with a system of many identical bosons) is entirely taken care of by the appropriate setting up of the Fock space in which the density operator $\rho$ resides. In fact an identical result is obtained for a system consisting of many identical fermions, in which case the commutation relations of the second quantized (or quantized field) operators are however changed as appropriate. Second, for second-quantized hamiltonians $H$ which commute with the total number operator $N$, the eigenvectors $\left|R_{j}\right\rangle$ of the density operator are simultaneous eigenvectors of $H$ and $N$ and, except for "accidental" degeneracy, no quantum interference effects exist involving different such eigenvectors. Of course, all energies and particle numbers contribute (with appropriate 
weights) to the grand-canonical thermal state described by $\rho$, and the actual determination of the appropriate eigenvectors involves the full complexity of the quantum manybody problem. Finally, if the many-body hamiltonian $H$ includes an external, confining one-body potential to represent a trap for the particles, one cannot take a thermodynamic limit by letting $\langle N\rangle \rightarrow \infty$ at constant density, since this is constrained by the trap. Correspondingly, in this case the grand-canonical formulation loses its equivalence to alternate (technically less friendly) treatments.

\subsection{Ideal Bose gas in a trap}

Non-interacting identical bosons in a trap, represented by an external, confining one-body potential $V(\vec{r})$, can be handled in terms of a very simple (possibly the only simple) application of the previous general results. What makes it simple is the absence of two- (or even possibly also otherwise many, like three-, etc.) atom interactions, which are generally present in real systems. Because of this, the qualification of this case as "ideal" has been entirely appropriate until late 2002, when a real "ideal" condensate of cesium atoms was produced and studied in the Austrian town of Innsbruck[2]. As will be discussed in Chapter 3 (see section 3.1), one remarkable experimental trick developed in connection with the alkali gases, which is particularly important in the case of cesium atoms, allows for the external control of the effective atom-atom interaction[3]. This is achieved by making judicious use of Zeeman displacements of atomic energy levels. Thus the control agent is just an external magnetic field, and it has permitted to tune the effective interactions among condensate cesium atoms to zero. The same trick had in fact been used before in the case of one of the rubidium isotopes $\left({ }^{85} \mathrm{Rb}\right)$ to obtain a real "ideal", non Bose-Einstein condensed thermal gas[4].

The case of an extended, non-interacting gas of identical bosons is of course equally simple to deal with in statistical mechanical terms, and became in fact a standard textbook case, in which one is able to take the thermodynamic limit and derive exact statistical mechanics results (see e.g. [6], section 1.9). In the case of the trapped ideal gas, the possibility of taking a thermodynamic limit (in the usual sense, at least) is excluded, so that in this case the results will bear marks which are specific of the grand-canonical formulation and which are moreover possibly artificial, such as particle number fluctuations. In spite of this, technical convenience has been decisive for the use of a grand-canonical framework for the trapped gas [7, 8].

The second-quantized hamiltonian for the noninteracting bosons in the trap can be written as

$$
H=\sum_{n} e_{n} a_{n}^{\dagger} a_{n}
$$

where the energies $e_{n}$ are the single-particle (with mass $M$ ) eigenvalues associated with the bound (normalized), stationary single-particle eigenfunctions $\varphi_{n}(\vec{r})$ of the trap

$$
\left[-\frac{\hbar^{2} \nabla^{2}}{2 M}+V(\vec{r})\right] \varphi_{n}(\vec{r})=e_{n} \varphi_{n}(\vec{r})
$$

and the $a_{n}, a_{n}^{\dagger}$ are boson annihilation and creation operators associated with the single-particle eigenfunctions $\varphi_{n}(\vec{r})$. They satisfy the standard Bose commutation relations

$$
\begin{gathered}
{\left[a_{m}, a_{n}^{\dagger}\right]=\delta_{m n},} \\
{\left[a_{m}, a_{n}\right]=\left[a_{m}^{\dagger}, a_{n}^{\dagger}\right]=0}
\end{gathered}
$$

and are related to the Bose field operators $\psi(\vec{r}), \psi^{\dagger}(\vec{r})$, which in turn satisfy the commutation relations

$$
\begin{gathered}
{\left[\psi(\vec{r}), \psi^{\dagger}\left(\vec{r}^{\prime}\right)\right]=\delta\left(\vec{r}-\vec{r}^{\prime}\right),} \\
{\left[\psi(\vec{r}), \psi\left(\vec{r}^{\prime}\right)\right]=\left[\psi^{\dagger}(\vec{r}), \psi^{\dagger}\left(\vec{r}^{\prime}\right)\right]=0,}
\end{gathered}
$$

through

$$
a_{n}=\int d^{3} r \varphi_{n}^{*}(\vec{r}) \psi(\vec{r}), \quad a_{n}^{\dagger}=\int d^{3} r \varphi_{n}(\vec{r}) \psi^{\dagger}(\vec{r})
$$

Note that the spin degree of freedom has been ignored in this formulation, which therefore applies either to spinless bosons or to bosons with spin but constrained to a definite magnetic substate, as it is in fact the case for magnetic traps. For non-interacting bosons of spin $S$ and spin-independent trap one-body potential one would still have to take into account the $2 S+1$ degeneracy of the single-particle states.

The number operator is in this setup given as

$$
N=\sum_{n} a_{n}^{\dagger} a_{n}
$$

so that the grand-canonical density operator (1.6) can be worked out to acquire the form of a product of singleparticle factors:

$$
\rho=\frac{e^{-\frac{1}{k_{B} T} \sum_{n}\left(e_{n}-\mu\right) a_{n}^{\dagger} a_{n}}}{\operatorname{Tr}\left(e^{-\frac{1}{k_{B} T} \sum_{n}\left(e_{n}-\mu\right) a_{n}^{\dagger} a_{n}}\right)}=\prod_{n} \frac{e^{-\frac{1}{k_{B} T}\left(e_{n}-\mu\right) a_{n}^{\dagger} a_{n}}}{\sum_{\nu_{n}=0}^{\infty} e^{-\frac{\nu_{n}}{k_{B} T}\left(e_{n}-\mu\right)}}=\prod_{n} \frac{e^{-\frac{1}{k_{B} T}\left(e_{n}-\mu\right) a_{n}^{\dagger} a_{n}}}{\left(1-e^{-\frac{e_{n}-\mu}{k_{B} T}}\right)^{-1}} .
$$

In the first step above the commutativity of the different terms in the exponents has been taken advantage of, while transition to the last form involved using the standard for- mula for the sum of a geometric series. Note that convergence of the series requires that $e_{n}-\mu>0$ for all $n$, which implies that the chemical potential $\mu$ has the smallest single- 
particle eigenvalue $e_{0}$ (say) as an upper bound. In what follows we define the energy scale so that $e_{0} \equiv 0$, so that one must have $-\infty<\mu<0$.

As mentioned in the preceding section, the value of $\mu$ is fixed by the subsidiary condition that the mean total number of particles (taken in the density operator (1.8)). To this effect we must evaluate

$$
\langle N\rangle=\operatorname{Tr}\left(\sum_{n} a_{n}^{\dagger} a_{n} \rho\right)=\sum_{n} \operatorname{Tr}\left(a_{n}^{\dagger} a_{n} \rho\right)=\sum_{n}\left\langle N_{n}\right\rangle
$$

where $\left\langle N_{n}\right\rangle$ is the mean number of particles in the singleparticle level $n$. This can be evaluated as

$$
\begin{aligned}
\left\langle N_{n}\right\rangle & =\operatorname{Tr}\left(a_{n}^{\dagger} a_{n} \rho\right)=\left(1-e^{-\frac{e_{n}-\mu}{k_{B} T}}\right) \operatorname{Tr}\left(a_{n}^{\dagger} a_{n} e^{-\frac{1}{k_{B} T}\left(e_{n}-\mu\right) a_{n}^{\dagger} a_{n}}\right)= \\
& =\left(1-e^{-\frac{e_{n}-\mu}{k_{B} T}}\right) \sum_{\nu=0}^{\infty} \nu e^{-\frac{1}{k_{B} T}\left(e_{n}-\mu\right) \nu}=-\left(1-e^{-\frac{e_{n}-\mu}{k_{B} T}}\right) \frac{d}{d \frac{\left(e_{n}-\mu\right)}{k_{B} T}} \sum_{\nu=0}^{\infty} e^{-\frac{1}{k_{B} T}\left(e_{n}-\mu\right) \nu}= \\
& =\frac{e^{-\frac{1}{k_{B} T}\left(e_{n}-\mu\right)}}{1-e^{-\frac{1}{k_{B} T}\left(e_{n}-\mu\right)}} \equiv \frac{z e^{-\frac{e_{n}}{k_{B} T}}}{1-z e^{-\frac{e_{n}}{k_{B} T}}},
\end{aligned}
$$

where, in the last step, the quantity $z=e^{\frac{\mu}{k_{B} T}}$, known as the fugacity, has been introduced. From the bound on the chemical potential $\mu$ and our choice of energy scale it follows that $0<z<1$. For a given temperature $T$, it is to be determined by the subsidiary condition regarding the total number of particles, since it determines the value of the chemical potential at that temperature. A particularly interesting quantity is the mean occupation of the lowest singleparticle state of the trap, which can be written in terms of the fugacity simply as

$$
\left\langle N_{0}\right\rangle=\frac{z}{1-z},
$$

which can essentially exhaust the total mean number $\langle N\rangle$ when the fugacity $z$ approaches its upper bound.

The actual determination of the chemical potential $\mu$, or of the fugacity $z$, involves the the single-particle spectrum $e_{n}$, and therefore requires further specification of the trap potential $V(\vec{r})$. A simple choice, which is moreover "realistic" for the current experimental setups, is that of an anisotropic harmonic potential

$$
V(\vec{r}) \rightarrow \frac{M}{2}\left(\omega_{1}^{2} x^{2}+\omega_{2}^{2} y^{2}+\omega_{3}^{2} z^{2}\right)
$$

which leads to the spectrum

$$
\begin{gathered}
e_{n} \rightarrow e_{n_{1} n_{2} n_{3}}=\hbar \omega_{1} n_{1}+\hbar \omega_{2} n_{2}+\hbar \omega_{3} n_{3}, \\
n_{i}=0,1,2, \ldots, \quad i=1,2,3 .
\end{gathered}
$$

For numerical purposes it is convenient to rewrite the final expression (1.9) for $\left\langle N_{n}\right\rangle$ using again the formula for the sum of a geometrical series as

$$
\left\langle N_{n}\right\rangle=z e^{-\frac{e_{n}}{k_{B} T}} \sum_{\nu=0}^{\infty} z^{\nu} e^{-\frac{\nu e_{n}}{k_{B} T}}=\sum_{\nu=1}^{\infty} z^{\nu} e^{-\frac{\nu e_{n}}{k_{B} T}}
$$

so that with this choice of trap potential one has

$$
\left\langle N_{n_{1} n_{2} n_{3}}\right\rangle=\sum_{\nu=1}^{\infty} z^{\nu} e^{-\nu n_{1} \frac{\hbar \omega_{1}}{k_{B} T}-\nu n_{2} \frac{\hbar \omega_{2}}{k_{B} T}-\nu n_{3} \frac{\hbar \omega_{3}}{k_{B} T}}
$$

The total mean number of particles is then[7]

$$
\langle N\rangle=\sum_{n_{1}, n_{2}, n_{3}=0}^{\infty}\left\langle N_{n_{1} n_{2} n_{3}}\right\rangle=\sum_{\nu=1}^{\infty} \sum_{n_{1}, n_{2}, n_{3}=0}^{\infty} \prod_{i=1}^{3} z^{\nu} e^{-\nu n_{i} \frac{\hbar \omega_{i}}{k_{B} T}}=\sum_{\nu=1}^{\infty} z^{\nu} \prod_{i=1}^{3} \frac{1}{1-e^{-\nu \frac{\hbar \omega_{i}}{k_{B} T}}}
$$

From eq. (1.11) one can numerically determine the fugacity $z$ (and hence the chemical potential $\mu$ ) as a function of the temperature. This completes the determination of the thermal quantum density operator $\rho$ for the ideal bosons in the harmonic trap. In the case of an isotropic trap, $\omega_{1}=\omega_{2}=\omega_{3}=\omega$ it reduces to 


$$
\langle N\rangle=\sum_{\nu=1}^{\infty} \frac{z^{\nu}}{\left(1-e^{-\nu \frac{\hbar \omega}{k_{B} T}}\right)^{3}} .
$$

Before turning to numerical examples, it is useful to use the general expressions themselves in order to characterize the Bose-Einstein condensation syndrome for the trapped ideal gas[8]. Returning for a moment to eq. (1.9), the total number of particles in excited single-particle states, $\langle N\rangle-\left\langle N_{0}\right\rangle$, can be written as

$$
\begin{aligned}
\left\langle N^{\prime}\right\rangle & =\langle N\rangle-\left\langle N_{0}\right\rangle=\sum_{n=1}^{\infty} \frac{e^{-\frac{1}{k_{B} T}\left(e_{n}-\mu\right)}}{1-e^{-\frac{1}{k_{B} T}\left(e_{n}-\mu\right)}}=\sum_{n=1}^{\infty} \frac{1}{e^{\frac{1}{k_{B} T}\left(e_{n}-\mu\right)}-1}< \\
& <\sum_{n=1}^{\infty} \frac{1}{e^{\frac{e_{n}}{k_{B} T}}-1} \equiv\left\langle N_{\max }^{\prime}\right\rangle .
\end{aligned}
$$

The inequality follows from the fact that $z<1$. Thus, if $\langle N\rangle>\left\langle N_{\max }^{\prime}\right\rangle$, it follows that at least $\langle N\rangle-\left\langle N_{\max }^{\prime}\right\rangle$ must occupy the lowest single-particle state $e_{0}$. The number $\left\langle N_{\max }^{\prime}\right\rangle$ is accordingly called the saturation number in [8]. For the isotropic harmonic trap, this saturation number can be well approximated in terms of a simple, closed expression. The trick is to observe by a simple counting procedure that in this special case the single-particle energy levels

$$
e_{n} \rightarrow \hbar \omega\left(n_{1}+n_{2}+n_{3}\right), \quad n=n_{1}+n_{2}+n_{3}
$$

are $(n+1)(n+2) / 2$-fold degenerate, so that one has

$$
\left\langle N_{\max }^{\prime}\right\rangle=\frac{1}{2} \sum_{n=1}^{\infty} \frac{(n+1)(n+2)}{e^{\frac{n \hbar \omega}{k_{B} T}}-1} .
$$

Now let $n \hbar \omega / k_{B} T \equiv x$ and $\hbar \omega / k_{B} T \equiv \xi$. Provided $\xi \ll 1$, the sum over $n$ can be well approximated by an integral as

$$
\left\langle N_{\max }^{\prime}\right\rangle \rightarrow \frac{1}{2 \xi^{3}} \int_{\xi / 2}^{\infty} \frac{(x+\xi)(x+2 \xi)}{e^{x}-1} d x .
$$

The integrands involving $x^{2}$ and $x$ in the numerator are regular as $x \rightarrow 0$, and thus one can estimate the corresponding integrals by replacing the lower integration limit $\xi / 2$ by zero in them. Then

$$
\int_{0}^{\infty} \frac{x^{2} d x}{e^{x}-1}=\int_{0}^{\infty} x^{2} \frac{e^{-x}}{1-e^{-x}} d x=\int_{0}^{\infty} \sum_{\nu=1}^{\infty} x^{2} e^{-\nu x} d x=\int_{0}^{\infty} \sum_{\nu=1}^{\infty} \frac{2}{\nu^{2}} e^{-\nu x} d x=\sum_{\nu=1}^{\infty} \frac{2}{\nu^{3}}=2 \zeta(3) .
$$

The sum of reciprocal powers has been expressed in terms of the Riemann zeta function $\zeta(s)$ (see e.g. ref. [9]) which for $s=3$ has the value $\zeta(3)=1.202 \ldots$ As for the integral involving $x$ in the numerator of the integrand one gets similarly

$$
3 \xi \int_{0}^{\infty} \frac{x d x}{e^{x}-1}=3 \xi \int_{0}^{\infty} \sum_{\nu=1}^{\infty} x e^{-\nu x} d x=3 \xi \sum_{\nu=1}^{\infty} \frac{1}{\nu^{2}}=3 \xi \times \zeta(2)=3 \xi \times 1.6449 \ldots
$$

which, for $\xi \ll 1$, amounts to just a small correction to the integral involving $x^{2}$ in the integrand. As for the remaining integral, involving the numerator $2 \xi^{2}$, the lower integration limit cannot be taken to zero due to the singular behavior of the integrand for $x \rightarrow 0$. It can however be evaluated as

$$
2 \xi^{2} \int_{\xi / 2}^{\infty} \frac{d x}{e^{x}-1}=2 \xi^{2} \int_{\xi / 2}^{\infty} \sum_{\nu=1}^{\infty} e^{-\nu x} d x=2 \xi^{2} \sum_{\nu=1}^{\infty} \frac{e^{-\nu \xi / 2}}{\nu}=-2 \xi^{2} \ln \left(1-e^{-\xi / 2}\right)
$$

Due to the presence of the factor $2 \xi^{2}$ this is again only a small correction to the first integral when $\xi \ll 1$. An adequate estimate of $\left\langle N_{\max }^{\prime}\right\rangle$ for the isotropic harmonic trap, when $x 1 \ll 1$, or equivalently $\hbar \omega \ll k_{B} T$, is therefore obtained by keeping just the $\zeta(3)$ contribution, leading to

$$
\left\langle N_{\max }^{\prime}\right\rangle \simeq \frac{\zeta(3)}{\xi^{3}}=1.202 \ldots\left(\frac{k_{B} T}{\hbar \omega}\right)^{3} .
$$

This formula is useful to set a temperature scale for the trapped ideal gas. In fact, the "critical" temperature $T_{c}^{\text {harm }}$ 
at which this estimate of $\left\langle N_{\max }^{\prime}\right\rangle$ becomes equal to the total mean number of particles $\langle N\rangle$, namely

$$
T_{c}^{\mathrm{harm}}=\frac{\hbar \omega}{k_{B}}\left(\frac{\langle N\rangle}{1.202 \ldots}\right)^{\frac{1}{3}}
$$

is that temperature below which the bosons start accumulating in the lowest single-particle state $e_{0}$. Note that $T_{c}^{\text {harm }}$ depends on the total mean number of bosons in the harmonic trap. The corresponding value of $\xi$ is $\xi_{c} \equiv \hbar \omega / k_{B} T_{c}=$ $(1.202 \ldots /\langle N\rangle)^{1 / 3}$, which guarantees that $\xi_{c} \ll 1$ and hence the reliability of the estimate if $\langle N\rangle$ is not too small.

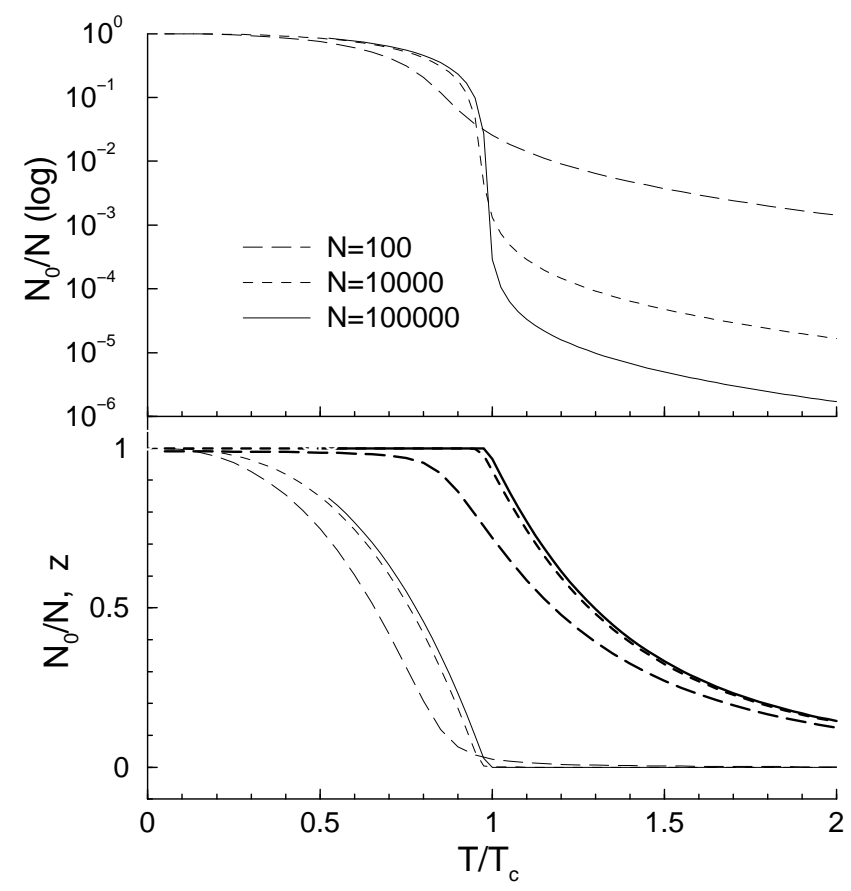

Figure 1.1. Occupation of the lowest single-particle state, $N_{0} / N$, and fugacity, $z$, (lowest graph, heavy lines) as a function of $T / T_{c}$, for the indicated values of the total mean number of particles in the isotropic harmonic trap. The upper graph is a logarithmic plot of $N_{0} / N$ to exhibit more clearly the behavior of this quantity in the transition region and above.

Results obtained by solving numerically eq. (1.12) for the fugacity $z$ and using eq. (1.10) to evaluate the occupancy of the lowest single-particle state $e_{0}$ as a function of temperature are shown for an isotropic harmonic trap in Fig.1.1. The temperature is plotted in units of the "critical" temperature $T_{c}^{\text {harm }}$ given by eq. (1.14) for the appropriate values of the total mean number of bosons. Supplying the values of the constants involved in this formula one gets

$$
T_{c}^{\mathrm{harm}}=4.5 N^{\frac{1}{3}} \nu(\mathrm{Hz}) \times 10^{-110} \mathrm{~K}
$$

where $\nu=\omega / 2 \pi$ is the trap frequency (in Hertz) and $T_{c}^{\text {harm }}$ is given in degrees Kelvin. Taking $\nu=200 \mathrm{~Hz}$ as a ballpark value for the trap frequency (which corresponds to an oscillator parameter $b \equiv(\hbar / M \omega)^{1 / 2} \simeq\left(20 / M_{\mathrm{amu}}\right)^{1 / 2} \times 10^{-3}$ $\mathrm{cm}, M_{\mathrm{amu}}$ being the boson mass in atomic mass units), one sees that $T_{c}^{\text {harm }}$ is about 40,200 and 400 nano-Kelvin for $\langle N\rangle$ equal to 100,10000 and 100000 respectively.

\subsection{1 “Quasi one-dimensional” harmonic trap}

Anisotropic traps can of course be dealt with going back to the more general eq. (1.11), instead of using its isotropic specialization, eq. (1.12). Here we consider as an example the case of a "quasi one-dimensional" trap[7], in which $\omega_{1} \simeq \omega_{2} \gg \omega_{3}=\omega$, with $\omega / 2 \pi$ in the $200 \mathrm{~Hz}$ ballpark. Thus the harmonic trap is very stiff in the transverse (1 and 2) directions, the corresponding excitation energies being much larger than that corresponding to longitudinal (direction 3) excitations.

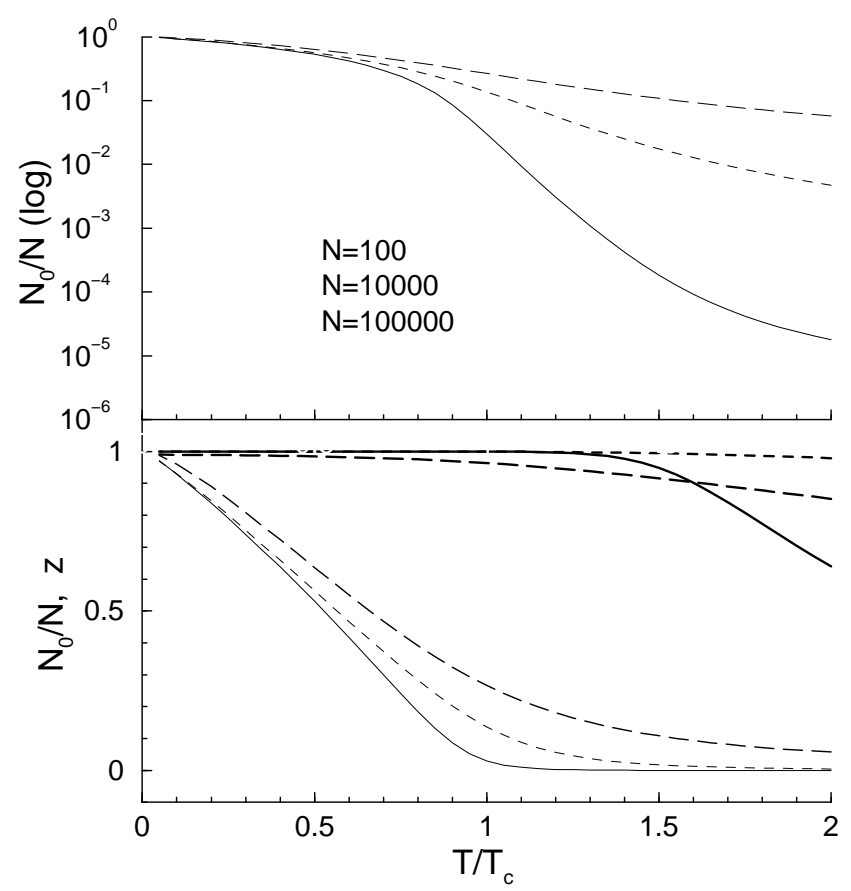

Figure 1.2. Similar to Fig. 1.1 for the quasi one-dimensional trapped ideal gas. The "critical" temperature $T_{c}$ is now given by eq. (1.16), for the indicated values of the total mean number of particles in a quasi one-dimensional harmonic trap with $\omega_{1} / \omega_{3}=\omega_{2} / \omega_{3}=10^{4}$. The fugacity and the condensed fraction have been obtained from eq. (1.11), taking transverse excitations into account.

In order to estimate the saturation number in this case, we neglect the contribution of transverse excitations in eq. (1.13), which in this way reduces to

$\left\langle N_{\max }^{\prime}\right\rangle \simeq \sum_{n=1}^{\infty} \frac{1}{e^{n \xi}-1} \rightarrow \frac{1}{\xi} \int_{\xi / 2}^{\infty} \frac{d x}{e^{x}-1}, \xi=\frac{\hbar \omega}{k_{B} T} \ll 1$

The integral appearing here has been evaluated before, so that one obtains 


$$
\begin{aligned}
\left\langle N_{\max }^{\prime}\right\rangle & \simeq-\frac{1}{\xi} \ln \left(1-e^{-\xi / 2}\right) \simeq \frac{1}{\xi} \ln \left(\frac{2}{\xi}\right) \\
& =\frac{k_{B} T}{\hbar \omega} \ln \left(\frac{2 k_{B} T}{\hbar \omega}\right) .
\end{aligned}
$$

The "critical temperature" $T_{c}^{\mathrm{qod}}$ for the quasi onedimensional, trapped free Bose gas is accordingly now determined by the equation

$$
\langle N\rangle=\frac{k_{B} T_{c}^{\mathrm{qod}}}{\hbar \omega} \ln \left(\frac{2 k_{B} T_{c}^{\mathrm{qod}}}{\hbar \omega}\right)
$$

and, due to the slow variation of the logarithmic factor with respect to the linear one, the condition $\xi \ll 1$ is again satisfied provided $\langle N\rangle$ is not too small, ensuring the validity of the estimate.

Numerical results for a trap with $\omega_{1} / \omega_{3}=\omega_{2} / \omega_{3}=10^{4}$ are shown in Fig. 1.2. This implies an aspect ratio of $1: 100$ for the ground state density, transverse excitation quanta being four orders of magnitude larger than the longitudinal quantum. As in the case of Fig. 1.1, the temperature is given in units of $T_{c}$, now determined from eq. (1.16). The fugacity $z$ and the fractional occupation of the lowest level, $\left\langle N_{0}\right\rangle /\langle N\rangle$ have been obtained by solving eq. (1.11) numerically, without neglecting the transverse excitations. This in fact affects especially the results for the fugacity, in the case with larger number of particles. For $\langle N\rangle=10^{5}, z$ remains within $1 \%$ of 1 in the entire range shown in the figure, when transverse excitations are neglected completely (as appropriate for the limit $\omega_{1} / \omega_{3}=\omega_{2} / \omega_{3} \rightarrow \infty$. One sees that the rise of the fractional occupation of the lowest level as the temperature is decreased below the "critical" temperature is qualitatively similar to that found for the spherically symmetric harmonic trap, but tends to a less abrupt, in fact linear character.

\subsection{Uniform vs. trapped ideal Bose gas}

The uniform ideal Bose-Einstein gas in three spatial dimensions, which was actually the case treated initially by Einstein in the 1920's, can be handled with the same tools used above in connection with the trapped gas. Here we replace eq. (1.7) by the free particle Schrdinger equation with periodic boundary conditions in volume $\mathcal{V}$, so that normalized single-particle wavefunctions and their respective single-particle energies are

$$
\varphi_{\vec{k}}(\vec{r})=\frac{1}{\sqrt{\mathcal{V}}} e^{i \vec{k} \cdot \vec{r}}, \quad e_{k}=\frac{\hbar^{2} k^{2}}{2 M}
$$

The corresponding boson creation and annihilation operators are written as $a_{\vec{k}}^{\dagger}$ and $a_{\vec{k}}$ respectively. Implementing the quantization volume as $\mathcal{V} \equiv L_{1} L_{2} L_{3}$ one has furthermore

$$
k^{2}=k_{1}^{2}+k_{2}^{2}+k_{3}^{2}=\left(\frac{2 \pi}{L_{1}}\right)^{2} n_{1}^{2}+\left(\frac{2 \pi}{L_{2}}\right)^{2} n_{2}^{2}+\left(\frac{2 \pi}{L_{3}}\right)^{2} n_{3}^{2}
$$

with $n_{i}=0, \pm 1, \pm 2, \pm 3, \ldots, i=1,2,3$. With these ingredients the relation determining the fugacity, which corresponds to eq. (1.11), reads

$$
\left\langle N_{(\mathcal{V})}\right\rangle=\sum_{n_{1}, n_{2}, n_{3}}\left\langle N_{n_{1} n_{2} n_{3}}^{(\mathcal{V})}\right\rangle=\sum_{n_{1}, n_{2}, n_{3}} \frac{1}{z^{-1} e^{\lambda_{T}^{2}\left(n_{1}^{2} / L_{1}^{2}+n_{2}^{2} / L_{2}^{2}+n_{3}^{2} / L_{3}^{2}\right)}-1}
$$

where $\left\langle N_{(\mathcal{V})}\right\rangle$ is the total mean number of bosons in volume $\mathcal{V}$ and

$$
\lambda_{T} \equiv \frac{2 \pi \hbar}{\sqrt{2 M k_{B} T}}
$$

is the de Broglie wavelength of a particle of mass $M$ and kinetic energy $k_{B} T$. It is interesting to write down the saturation number for this case. It is given by

$$
\left\langle N_{(\mathcal{V}) \max }^{\prime}\right\rangle=\sum_{n_{i}}{ }^{\prime} \frac{1}{e^{\lambda_{T}^{2}\left(n_{1}^{2} / L_{1}^{2}+n_{2}^{2} / L_{2}^{2}+n_{3}^{2} / L_{3}^{2}\right)}-1}
$$

where the term with $n_{1}=n_{2}=n_{3}=0$ is excluded form the sum.

In order to study the uniform gas in three dimensions, set $L_{1}=L_{2}=L_{3}=L$ and use the appropriate density of states to approximate this sum in terms of an integral, for $\lambda_{T} / L \ll 1$, as

$$
\left\langle N_{\left(L^{3}\right) \max }^{\prime}\right\rangle \rightarrow \frac{L^{3}}{\lambda_{T}^{3}} \int^{\prime} \frac{d^{3} x}{e^{x^{2}}-1}=\frac{4 \pi L^{3}}{\lambda_{T}^{3}} \int_{\lambda_{T} / 2 L}^{\infty} \frac{x^{2} d x}{e^{x^{2}}-1} .
$$

This shows that one can define in this case a saturation density

$$
\rho_{\max }^{\prime} \equiv\left\langle N_{\left(L^{3}\right) \max }^{\prime}\right\rangle / L^{3}
$$

which remains finite in the thermodynamic limit $\left\langle N_{\left(L^{3}\right)}\right\rangle \rightarrow$ $\infty, L \rightarrow \infty$ with constant total mean density $\left\langle N_{\left(L^{3}\right)}\right\rangle / L^{3}$. The limiting value of the integral can be obtained in terms of a series of standard gaussian integrals as 


$$
\int_{0}^{\infty} \frac{x^{2} d x}{e^{x^{2}}-1}=\int_{0}^{\infty} x^{2} \sum_{\nu=1}^{\infty} e^{-\nu x^{2}} d x=\frac{\sqrt{\pi}}{4} \sum_{\nu=1}^{\infty} \frac{1}{\nu^{3 / 2}}=\frac{\sqrt{\pi}}{4} \zeta\left(\frac{3}{2}\right)=\frac{\sqrt{\pi}}{4} \times 2.612 \ldots
$$

so that the saturation density becomes in the thermodynamic limit

$$
\rho_{\max }^{\prime} \rightarrow 2.612 \cdots \times \frac{\pi^{3 / 2}}{\lambda_{T}^{3}}=2.612 \cdots \times\left(\frac{2 \pi M k_{B} T}{4 \pi^{2} \hbar^{2}}\right)^{\frac{3}{2}} .
$$

The temperature at which the saturation density equals the total density is the critical temperature of the uniform gas, given therefore by (cf. eq.(1.14))

$$
T_{c}=\frac{2 \pi \hbar^{2}}{M k_{B}}\left(\frac{\left\langle N_{\left(L^{3}\right)}\right\rangle}{2.612 \ldots L^{3}}\right)^{\frac{2}{3}}=\frac{2 \pi \hbar^{2}}{M k_{B} L^{2}}\left(\frac{\left\langle N_{\left(L^{3}\right)}\right\rangle}{2.612 \ldots}\right)^{\frac{2}{3}}
$$

This implies that in an extended, uniform system at a temperature smaller that the critical temperature $T_{c}$ given by (1.19), there will be a finite fraction of the total density which is associated with occupation of the zero momentum state by the bosons. This is the standard (and original!) Bose-Einstein condensation phenomenon.

Furthermore, it is interesting to compare in some detail the expressions (1.19) and (1.14) for the critical temperature, which correspond respectively to the uniform ideal bose gas and to the ideal bose gas confined in the isotropic harmonic trap. They have clearly a similar structure, the critical temperature being given in both cases in terms of a single-particle energy scale divided by the Boltzmann constant times a fractional power of the mean number of particles. In the case of the uniform gas, this number refers to the quantization volume $\mathcal{V}=L^{3}$, which plays the role of a system "size" and also determines the relevant single-particle energy scale $\hbar^{2} / M L^{2}$. Analogously, in the case of the harmonic trap the single-particle energy scale $\hbar \omega$ defines a "size" scale through the oscillator parameter $b=\sqrt{\hbar / M \omega}$, the single-particle energy scale being also proportional to the inverse square of the size scale.

In both cases, therefore, the factor consisting of the energy scale divided by the Boltzmann constant is proportional to the inverse square of the size scale of the system. In the case of the uniform gas this size dependence is compensated by the factor involving the two thirds power of the mean number of particles when taking the thermodynamic limit, but not so in the case of the harmonic trap, where the corresponding factor involves the one third power of the mean number of particles. As a result of this, if one enlarges the trap by reducing its frequency $\omega$ and at the same time increases the number of particles so that the density parameter $\langle N\rangle / b^{3}$ is kept constant, one is left with a system with a lower critical temperature $T_{c}^{\text {harm }}$, which in fact approaches zero as $\omega^{1 / 2}$. In this way one sees that condensation of free bosons in the harmonic trap is a strictly finite system phenomenon $^{3}$.

An obvious question at this point concerns the ultimate origin of the different behaviors of the two types of system. It can be answered, if somewhat technically, in terms of the basic dynamic ingredients entering in the two statistical calculations, namely the single-particle spectra, including degeneracy factors. In the case of the harmonic trap the level spacing is constant and the degeneracy increases quadratically with quantum number, while for the free gas both the level spacing (the spectrum having been discretized by periodic boundary conditions) and the degeneracy increase quadratically, effectively inhibiting thermal excitations. In order to test this interpretation one may conceive an anharmonic, symmetric trap with single-particle spectrum

$$
e_{n_{1}, n_{2}, n_{3}}=\hbar \omega\left(n_{1}^{2}+n_{2}^{2}+n_{3}^{2}\right)
$$

the corresponding eigenfunctions being ordinary harmonic oscillator eigenfunctions. Then the relevant size parameter $b$ is proportional to $\omega^{-1 / 2}$, and one finds that $T_{c}$ approaches a non-zero limit when $\langle N\rangle$ and $b$ are increased with constant $\langle N\rangle / b^{3}$.

A difference of another nature between the gas in the harmonic trap and the free gas relates to the fact that in the latter case the condensate density $\left\langle N_{0}\right\rangle / L^{3}$ is spatially uniform (as also is the total density), while in the trap it has the spatial distribution of the trap ground-state wavefunction, $\left|\varphi_{0}(\vec{r})\right|^{2}$, which is smaller than the total spatial distribution of the gas for $T>0$. This is due to the fact that in the case of the free gas the single-particle ground state is the zero momentum state, which leads to the statement that the Bose-Einstein condensation of the free gas occurs "in momentum space", unlike the situation in the case of the trapped gas, in which spatial segregation of the condensed fraction takes place. This latter effect has been in fact used as a signature for the occurrence of condensation in trapped atomic gases. Note that a similar segregation occurs also in

\footnotetext{
${ }^{3}$ The situation is if fact somewhat more complicated than just stated due to the fact that in order to write eq. (1.14) "small corrections" involving higher powers of the small quantity $\xi$ were neglected. A more complete appraisal of the situation can be obtained by going back to the saturation number $\left\langle N_{\text {max }}^{\prime}\right\rangle$ for the symmetric harmonic trap including the neglected contributions. As $b$ is increased they remain smaller then the dominant contribution, but scale differently with $b$. Thus, in the case of the trapped gas, strictly speaking the critical temperature is not an homogeneous function of the scale parameter.
} 
the case of the hypothetical anharmonic trap. Since this allows for condensation in the "large system limit", the spatial segregation effect cannot be related to the restriction to finite systems of the condensation in the harmonic trap.

\subsubsection{One-dimensional free gas}

We finally illustrate the dependence on the spatial dimensionality of the condensation phenomenon by considering the case of a uniform one-dimensional ideal gas. To this effect we return to eq. (1.17) and make the system highly anisotropic by letting $L^{3}=L$ and $L_{1} / L=L_{2} / L \gg 1$. If these ratios are so large that transverse excitations can be neglected (i.e., the restriction $n_{1}=n_{2}=0$ applies), one gets, writing $n_{3}=n$,

$$
\left\langle N_{(L)}\right\rangle=\sum_{n}\left\langle N_{n}^{(L)}\right\rangle=\sum_{n} \frac{1}{z^{-1} e^{n^{2} \lambda_{T}^{2} / L^{2}}-1}
$$

The saturation number is given by the same expression with the $n=0$ term omitted and with $z=1$. For small $\lambda_{T} / L$ it can be estimated from the integral

$$
\left\langle N_{(L) \max }^{\prime}\right\rangle=\frac{L}{\lambda_{T}} \int_{\lambda_{T} / 2 L}^{\infty} \frac{2 d x}{e^{x^{2}}-1} \rightarrow \frac{4 L^{2}}{\lambda_{T}^{2}}=k_{B} T\left(\frac{4 \pi^{2} \hbar^{2}}{8 M L^{2}}\right)^{-1}, \quad \lambda_{T} / L \ll 1
$$

showing that the corresponding critical temperature goes to zero as one approaches the thermodynamic limit. The corresponding result for the (quasi) one-dimensional harmonic trap is given by eq. (1.15) which, according to eq. (1.16), in fact prescribes an even slightly faster decrease of the critical temperature when $\langle N\rangle$ and $b$ are increased with constant $\langle N\rangle / b$.

\subsection{Relevant parameters and orders of mag- nitude}

According to eq. (1.19), the condensation of a homogeneous system of ideal bosons of mass $M$ can be characterized in terms of two length parameters. One of them is related to the mean interparticle spacing, and can be taken as the inverse one-third power of the particle density, $\rho_{P}^{-1 / 3} \equiv L /\left\langle N_{\left(L^{3}\right)}\right\rangle^{1 / 3}$. The second parameter can be taken as the thermal wavelength (1.18) associated with the critical temperature $T_{c}, \lambda_{T_{c}}$. In fact, eq. (1.19) can be expressed in terms of the dimensionless quantity $\rho_{P} \lambda_{T_{c}}^{3}$ as

$$
\rho_{P} \lambda_{T_{c}}^{3}=2.612 \ldots \times \pi^{3 / 2} .
$$

It has become customary to use, instead of $\lambda_{T}$ defined as in eq. (1.18), a so called de Broglie wavelength $\lambda_{d B} \equiv$ $\lambda_{T} / \sqrt{\pi}$, in terms of which one has

$$
\rho_{P} \lambda_{d B}^{3}=2.612 \ldots, \quad T=T_{c} .
$$

This latter quantity is often referred to as the phase space density for the homogeneous gas, and its critical value is often used as a ballpark to characterize experimentally the conditions to achieve Bose-Einstein condensation. Note that this phase space density becomes larger as the density is increased and as the temperature is lowered, so that the critical value represents a minimum value to be attained if condensation is to be achieved. This is at the root of a customary pictorial interpretation of the Bose-Einstein condensation as a "collective quantum effect" in which the allowed degree of localization of different particles, represented by $\lambda_{d B}$, becomes smaller that the mean inter-particle distance, represented by $\rho_{P}^{-1 / 3}$.

The use of the critical phase space density in order to characterize the onset of condensation of the trapped gas is in principle unwarranted. Even the use of the thermal de Broglie wavelength must be used with caution in view of the spatial quantization restrictions due to the trap potential. If, however, one invokes semiclassical arguments to treat a sufficiently extended and dense trapped system as being "locally uniform" it is easy to derive the semiclassical approximate relation[10]

$$
\rho_{P}(0) \lambda_{d B}^{3} \simeq 2.612 \ldots, \quad T=T_{c}
$$

where now $\rho_{P}(\vec{r})$ is local density of the trapped gas at position $\vec{r}, \rho_{P}(0)$ corresponds to the peak value of the local density, assumed to be located at the origin of the adopted reference system. This relation is in fact used experimentally in order to characterize the ballpark values of the parameters relevant to the onset of condensation in traps, in substitution for (e.g.) eq. (1.14).

Finally, it is useful to quote expressions for the oscillator parameter $b$ and for the de Broglie wavelength $\lambda_{d} B$ in practical units. One has

$$
\begin{aligned}
& b \simeq \sqrt{\frac{40}{M(\mathrm{amu}) \nu(\mathrm{Hz})}} \times 10^{-2} \mathrm{~cm} \text { and } \\
& \lambda_{d B}=\frac{1.747}{\sqrt{M(\mathrm{amu}) T(\mu \mathrm{K})}} \times 10^{-4} \mathrm{~cm}
\end{aligned}
$$


where $M(\mathrm{amu})$ is the mass of the atoms in atomic mass units, $\nu(\mathrm{Hz})$ is the frequency of the harmonic trap in hertz and $T(\mu \mathrm{K})$ is the temperature in micro-Kelvin.

\section{Non-ideal dilute Bose gas}

Interactions among atoms constitute an essential ingredient of nature as we see it, manifested in the existence of molecules, from simple to very complex, liquid and solid phases both of pure elements and compounds of various sorts, etc. In the comparatively very simple systems consisting of very cold, dilute, trapped atomic gases which concern us here, atom-atom interactions also play an essential role, to begin with, in the very process trough which they are produced. In fact, an important step towards attaining the conditions for the formation of Bose-Einstein condensates is the so called evaporative cooling. In this process the trap is set up in such a way that the "fastest" (more energetic) atoms are allowed to escape, leading to a reduction in temperature through a re-establishment of thermal equilibrium. Both the establishment of an initial velocity distribution of an "universal" (thermal) type and its colder re-establishment following selective depletion of the initial one require the action of atom-atom interactions. In this way, the recently achieved "ideal" condensate of cesium atoms could only be produced by making use of a trick allowing the atom-atom interaction to be experimentally "tuned". Thus, in order to produce the "ideal" condensate, the interaction strength is first set to a convenient value for the cooling process; $a f$ ter the (non-ideal!) condensate is produced, the interaction strength is set to zero, rendering it "ideal"[2].

The natural ubiquity of bound complex molecular structures clearly indicates, moreover, that interactions between atoms are in general extremely rich and complex and also that they must contain enough attractive effects in order to account for the observed binding. As was put decades ago by Victor Weisskopf, forces between atoms can be seen as being much "stronger" than the strong forces between nucleons, in the sense that the latter have a single, almost spherically symmetric two-body bound state (the deuteron), whereas a typical two-atom system has a huge number of spatially very complex molecular bound states.

However, in a gaseous phase and at very low temperatures the complexities of the atom-atom interaction are rendered largely ineffective. Considering an atom-atom collision event at values of the relative momentum corresponding to kinetic energies $k_{B} T$ with $T$ in the sub-micro Kelvin range, the classical turning point for the centrifugal barrier corresponding to angular momentum $l$ occurs at a relative separation $r_{l}$ given by

$$
r_{l}=\sqrt{\frac{\hbar^{2} l(l+1)}{2 M k_{B} T}}=\sqrt{\pi l(l+1)} \lambda_{d B}(T)
$$

which, according to (1.21), is in the $10^{-4} \mathrm{~cm}$ range for $l=1$. Since this is more than three orders of magnitude larger than the sizes of molecules (or of atomic cross sections, typically associated to linear dimensions of up to a few hundred Bohr radii), scattering processes are under these conditions effectively restricted to $s$-wave elastic scattering, and are completely characterized by the corresponding scattering phase-shift. This of course effectively restricts the immediate correlation capabilities of the interatomic forces to nuclear simplicity, or even below, at least as long as one remains in a cold, gaseous phase.

The attractive character of the interatomic forces, responsible for the existence of molecules and of condensed (e.g. liquid and solid) phases at low temperatures signals however that the gaseous, non-ideal many-boson systems which undergo Bose-Einstein condensation are in fact not approaching their ground-states as they are cooled, but are rather approaching some excited state, meta-stable at best, raising the important question of the degree of stability of this particular, and rather peculiar kind of phase. Qualitatively, the meta-stability is enhanced if the cold gas is $d i$ lute, in the sense that three-body collisions that are the only efficient way of disposing of the surplus energy (a process known as three-body recombination) have a very small probability in comparison with with the two-body elastic collisions responsible for establishing and maintaining thermal equilibrium.

\subsection{Simple effective interaction}

The center of mass scattering cross-section for scattering of two particles at low enough relative energy $\hbar^{2} k^{2} / 2 \mu, \vec{k}$ being the relative momentum and $\mu$ being the reduced mass, so that only $s$-waves are affected by the interparticle potential, can be expressed in terms of the $s$-wave phase shift $\delta_{0}(k)$ as

$$
\frac{d \sigma}{d \Omega}=\frac{\sin ^{2} \delta_{0}(k)}{k^{2}} \quad \stackrel{k \rightarrow 0}{\longrightarrow} a^{2}
$$

where $a$ has dimensions of length. There is no dependence of the cross-section on the center of mass scattering angle. There are at least two different lessons to be learned from this simple fact. First, very low energy scattering is largely insensitive to the detailed nature of the interparticle potential, as its relevant effects can be characterized by a single parameter. Second, the $s$-wave scattering phase shift $\delta_{0}(k)$ is not very adequate for this purpose in view of the denominator $k^{2}$, which requires $\sin \delta_{0}(k)$ to vanish linearly when $k \rightarrow 0$ for any finite value of the cross section. 
In order to circumvent this inadequacy of the parameterization in terms of the phase shift one introduces thescattering length $a$ defined as

$$
\lim _{k \rightarrow 0} \frac{k}{\sin \delta_{0}(k)} \equiv-\frac{1}{a}
$$

which therefore coincides (with a defined sign) with the length parameter introduced in (2.1). In order to refine the description to describe the dependence of the low-energy cross-section with the relative momentum, this last definition is interpreted as the first term of an expansion, in powers of $k$, of the quantity $k \cot \delta_{0}(k)$. One can show (see e.g. [1], section 5.1.6) that the linear term of the expansion vanishes identically, so that it can be generally written as

$$
k \cot \delta_{0}(k)=-\frac{1}{a}+\frac{r_{0}}{2} k^{2}+\ldots
$$

known as the effective range expansion, and the coefficient $r_{0}$ of the term quadratic in $k$ is called the effective range. With the use of this expansion, the low energy scattering can be described in terms of just the two parameters $a$ and $r_{0}$, so that all potentials having given values of these parameters produce the same scattering at low energies. An important point to be kept in mind is that the effective range expansion gives a parameterization of the scattering amplitude (since it gives the dependence on $k$ of the only relevant phase shift $\left.\delta_{0}(k)\right)$. Therefore, in order to obtain the scattering length for some given two-body potential one has to solve a two-body scattering problem. For instance, for a hard-core repulsive potential or radius $a$ the $s$-wave phase shift is $\delta_{0}(k)=-k a$. This is obtained by solving the appropriate Schrdinger equation with scattering boundary conditions. From this result one finds that the scattering length is equal to the hard-core radius, and that the effective range $r_{0}$ vanishes. Note that the parameters of the effective range expansion are finite, even though the potential itself is not, and neither are perturbative amplitudes, such as the Born amplitude, for this particular potential. This can be readily understood if one recalls that the scattering amplitude can in general be written in terms of the exact scattering solution of the two-body Schrdinger equation $\left|\phi_{\vec{k}}^{+}\right\rangle$as (see [1], p. 512)

$$
f_{\vec{k} \rightarrow \vec{k}^{\prime}}=-\frac{M}{2 \pi \hbar^{2}}\left\langle\vec{k}^{\prime}|V| \phi_{\vec{k}}^{+}\right\rangle .
$$

In the case of the hard-core potential this wavefunction vanishes inside the repulsive core, giving a finite result for the bracket which determines the scattering amplitude.

This feature of the scattering amplitude has been used to a considerable extent in connection with the many-body problem involving two-body interaction potentials which are possibly singular (e.g, including hard cores). The basic trick consists in replacing the potential itself by an effective operator which incorporates the correlation effects due to the potential which cannot be treated perturbatively. It is known as Brueckner's theory and has been originally developed in connection with the nuclear (fermionic) manybody problem[11], but has been immediately applied also to the hard-sphere Bose-Einstein gas[12]. In the case of the two-body scattering problem this is accomplished simply by the transition operator $T\left(k^{2}\right)$ having the property (see [1], p. 537)

$$
\left\langle\vec{k}^{\prime}|V| \phi_{\vec{k}}^{+}\right\rangle=\left\langle\vec{k}^{\prime}\left|T\left(k^{2}\right)\right| \vec{k}\right\rangle .
$$

As indicated by the notation, this is in general an energydependent operator, requiring considerable technical labor when used in more elaborate many-body calculations[12].

A standard approach to the effective interaction currently used in connection with cold, dilute Bose-Einstein atomic gases can be seen as a combination of the transition operator trick with the effective range expansion. One replaces the actual atom-atom interaction by a "contact" (zerorange) two body effective interaction which can be represented as

$$
v_{\mathrm{eff}}\left(\vec{r}_{1}, \vec{r}_{2}\right)=\frac{4 \pi \hbar^{2} a}{M} \delta\left(\vec{r}_{1}-\vec{r}_{2}\right)
$$

where $a$ stands for the scattering length characterizing the low energy atom-atom scattering. This fact alone indicates immediately that this effective interaction is in fact related to the scattering amplitude, and must therefore be seen as belonging to the hierarchical level of the transition operator, rather than to that of the two-body potential. In fact, when $v_{\text {eff }}$ is treated in the first Born approximation, it reproduces the low-energy limit of the two-body collision crosssection, written in terms of the scattering amplitude $a$. The many-body effective hamiltonian for a system of $N$ identical bosons in an external trap represented by the one body external potential $V(\vec{r})$ is therefore written as

$$
H_{\mathrm{eff}}=\sum_{i=1}^{N}\left(\frac{p_{i}^{2}}{2 M}+V\left(\vec{r}_{i}\right)\right)+\frac{1}{2} \sum_{i \neq j=1}^{N} \lambda \delta\left(\vec{r}_{i}-\vec{r}_{j}\right) \quad \text { with } \quad \lambda \equiv \frac{4 \pi \hbar^{2} a}{M}
$$

or alternatively, in second-quantized form, as 


$$
\begin{aligned}
H_{\mathrm{eff}} & =\int d^{3} r \mathcal{H}(\vec{r}) \\
\mathcal{H}(\vec{r}) & =\psi^{\dagger}(\vec{r})\left(-\frac{\hbar^{2} \nabla^{2}}{2 M}+V(\vec{r})\right) \psi(\vec{r})+\frac{\lambda}{2} \psi^{\dagger}(\vec{r}) \psi^{\dagger}(\vec{r}) \psi(\vec{r}) \psi(\vec{r})
\end{aligned}
$$

where $\mathcal{H}(\vec{r})$ is the hamiltonian density, written in terms of the field operators $\psi^{\dagger}(\vec{r}), \psi(\vec{r})$ introduced in page 1106 . Note again that spin degrees of freedom have been ignored. This is of course correct for spinless identical bosons, but applies also to the case of bosons with non-zero spin when they are constrained to just one magnetic substate, as it is the case for magnetic traps. Other situations allowing for different spin states will be discussed below.

The two-body interaction term included in the effective hamiltonian (2.4), or (2.5), has the peculiar feature that its attractive or repulsive character depends on the sign of the scattering length $a$ being negative or positive, respectively. A well known result of effective range theory is that the scattering length of a purely repulsive potential is always positive. However, if the potential is not purely repulsive, or even if it is purely attractive, the scattering length can in general have either one of the two signs. Thus, from the fact that the scattering length is positive one cannot draw the conclusion that the potential is repulsive. Actually, even though the atom-atom interaction involves enough attraction to support a large number of molecular bound states, it may possibly be associated with a positive scattering length, in which case it is represented by a repulsive effective interaction when the prescription (2.3) is used. One clear implication of this is that a simple prescription such as eq. (2.3) cannot possibly be adequate for the treatment of more demanding properties of the many-boson system, such as instabilities related to three-body recombination processes, to cite just one and very obvious example. It is presently widely and successfully used mainly in connection with approximate treatments of non-ideal, dilute Bose-Einstein condensed systems, of their elementary excitations and of several of their collective properties, as discussed in continuation and in the following chapters.

The inclusion of the effective two-body interaction $v_{\text {eff }}$ also introduces a new length parameter into the many-boson system, associated with the effective range of the interaction, which can be identified with the scattering length $a$. The relation of this parameter to the mean interparticle distance $\rho_{P}^{1 / 3}, \rho_{P}$ being the particle density, allows for a quantitative characterization of the "diluteness" of the system in terms of the dimensionless quantity $\rho_{P} a^{3}$. The quantity $a^{3}$ represents an interaction volume, so that $\rho_{P} a^{3}$ corresponds to the average number of particles in the interaction volume.
Typical densities of gaseous condensates currently obtained are in the ballpark of $\rho_{P} \sim 10^{15} \mathrm{~cm}^{-3}$. Using $a \simeq 100 r_{B}$ also as a ballpark value for the scattering length, $r_{B}$ being the Bohr radius, one obtains $\rho_{P} a^{3} \sim 10^{-4}$. This quantity is therefore apt to be used as small expansion parameter when one deals with these systems. This is to be contrasted with the situation of liquid ${ }^{4} \mathrm{He}$, for which $\rho_{P} a^{3}>1$.

\subsection{Effective mean-field (Gross-Pitaevski) ap- proximation}

The simplest, but still extremely useful use of the effective hamiltonian (2.4) (or its second-quantized version (2.5)) is the derivation of a "mean field approximation" to the BoseEinstein condensed state of the non-ideal, dilute gas. This can be obtained variationally by looking for extrema of the energy functional $\left\langle\Phi\left|H_{\text {eff }}\right| \Phi\right\rangle$ using an ansatz for $|\Phi\rangle$ of the Hartree type. In wavefunction language, appropriate for use in connection with the form (2.4) of the effective hamiltonian, this ansatz is

$$
\Phi\left(\vec{r}_{1}, \ldots, \vec{r}_{N}\right)=\prod_{i=1}^{N} \varphi\left(\vec{r}_{i}\right)
$$

where $\varphi(\vec{r})$ is a normalized single-particle wavefunction to be determined. Note that all bosons are in the same singleparticle state, so that the symmetrization requirement is fulfilled automatically. Alternatively one can use the secondquantized form (2.5) of the effective hamiltonian in together with the ansatz

$$
|\Phi\rangle=\frac{1}{\sqrt{N !}} a^{\dagger N}|0\rangle
$$

where the creation operator $a^{\dagger}$ is expressed in terms of the field operator $\psi^{\dagger}(\vec{r})$ as

$$
a^{\dagger} \equiv \int d^{3} r \varphi(\vec{r}) \psi^{\dagger}(\vec{r})
$$

Calculations are in this case made easier by noting the commutation relations

$$
\left[a, a^{\dagger}\right]=1 \quad \text { and } \quad\left[\psi(\vec{r}), a^{\dagger}\right]=\varphi(\vec{r})
$$

which follow directly from the normalization of the singleparticle wavefunction $\varphi(\vec{r})$ and from the commutation rela- 

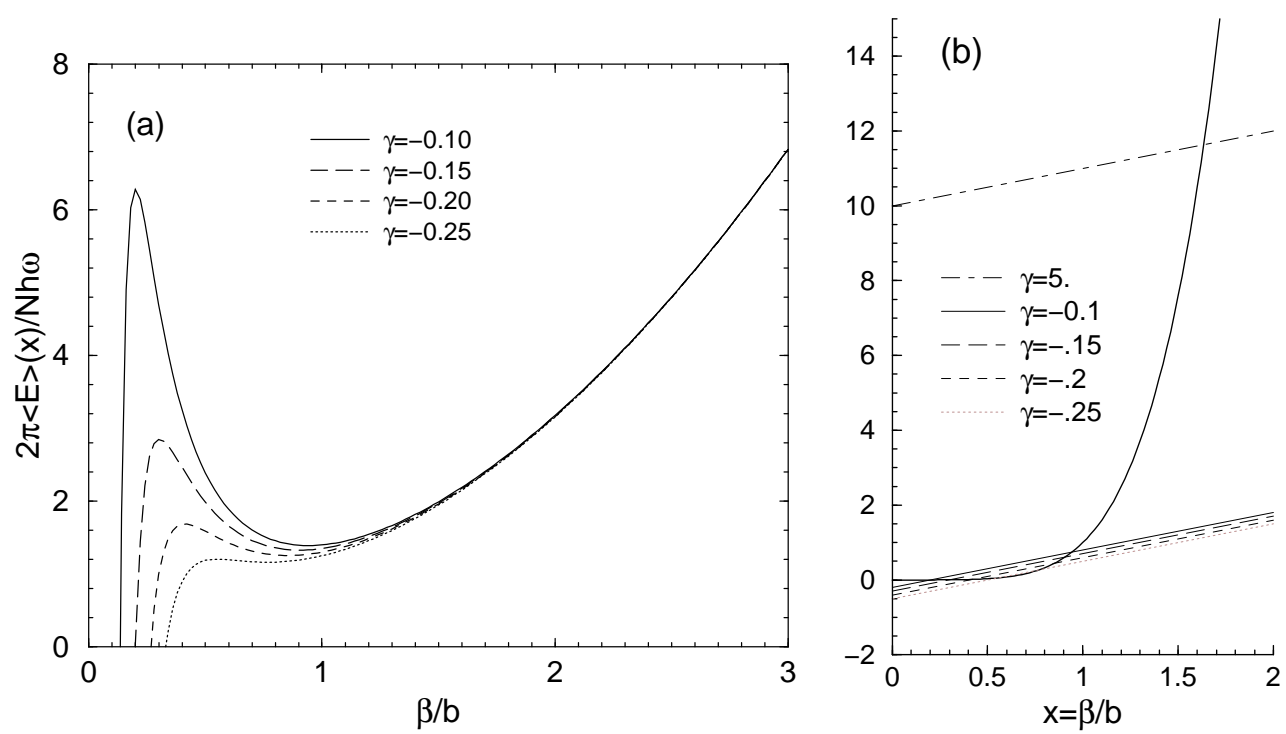

Figure 2.1. (a) Energy functional per particle for an isotropic harmonic trap in units of $\hbar \omega$, with gaussian ansatz for the single-particle wavefunction, plotted as a function of the gaussian width parameter $\beta$. The various curves correspond to the indicated values of the dimensionless parameter $\gamma=(N-1) a / \sqrt{2 \pi} b$. (b) Solutions of equation (2.10) for the indicated values of $\gamma$. The straight lines correspond to the left-hand side of the equation, and the $\beta$ values of the solutions (extrema of $\langle E\rangle(x)$ ) are determined by their intercepts with the fifth order parabola representing the right hand side.

Now it is easy to see that this functional does not have a lower bound when the effective two-body potential is attractive, i.e., when $\lambda<0$, which amounts to a negative scattering length $a$. To this effect it is sufficient to make a gaussian ansatz for the single-particle wavefunction

$$
\varphi(\vec{r}) \rightarrow\left(\frac{1}{\beta \sqrt{\pi}}\right)^{\frac{3}{2}} e^{-r^{2} / 2 \beta^{2}}
$$

and note that the kinetic energy contribution is positive and proportional to $\beta^{-2}$, the contribution of the term involving the one-body potential is just the gaussian average of a supposedly non-singular function of $\vec{r}$, while the contribution from the two-body potential has the sign of $\lambda$ and is propor- tional to $\beta^{-3}$. Therefore, if $\lambda<0$ the value of the energy functional can be made arbitrarily negative by choosing a sufficiently small value of $\beta$. This indicates the collapse of the system when the scattering length is negative.

Remarkably, this fact that has been observed experimentally[2, 13], with some nontrivial characteristics which are quantitatively consistent with properties of the energy functional (??). The relevant properties can be spotted by actually evaluating the energy functional with the gaussian ansatz, assuming an isotropic harmonic trap with angular frequency $\omega$, so that its size parameter is the oscillator parameter $b=\sqrt{\hbar / M \omega}$. This is a straightforward exercise giving as result

$$
\mathcal{E}[\varphi(\vec{r})] \rightarrow\langle E\rangle(\beta)=N \hbar \omega\left[\frac{3}{4}\left(\frac{b^{2}}{\beta^{2}}+\frac{\beta^{2}}{b^{2}}\right)+\frac{(N-1) a}{\sqrt{2 \pi} b} \frac{b^{3}}{\beta^{3}}\right]
$$

where $a$ is the scattering length appearing in the effective two-body potential parameter $\lambda$. Fig. 2.1 shows a plot of the mean energy per particle (in units of $\hbar \omega$ ) as a function of $\beta / b$ for some negative values of the dimensionless parameter

$$
\gamma \equiv \frac{(N-1) a}{\sqrt{2 \pi} b} .
$$

The "collapse" situation is revealed by the mean energy per particle going to minus infinity as $\beta / b \rightarrow 0$, but when $\gamma$ is not negatively too large, this quantity also has a minimum, separated from the region of collapse by a kinetic energy "barrier" which decreases as $\gamma$ becomes more negative un- til it disappears for $\gamma$ below about 0.25 . For given trap frequency and (negative) scattering length, this implies the prediction of "meta-stable" solutions for $N$ smaller than some critical number of particles $N_{c}$ in the trap, which depends on the values of $a$ and $b$. The gaussian approximation to the single-particle wavefunction for the meta-stable state can be found from the extremum condition obtained by by setting the derivative of (2.8) with respect to $x \equiv \beta / b$ equal to zero. One finds

$$
\frac{1}{2 x^{3}}+\frac{\gamma}{x^{4}}=\frac{x}{2} \quad \text { or } \quad x+2 \gamma=x^{5}, \quad x>0 .
$$


The graphical solution of this last equation is shown in Fig. 2.1 (b) for the same negative values of $\gamma$ shown in the part (a) of the figure. Also included is the case of a repulsive effective two-body interaction with $\gamma=5$. The value of $\beta$ which minimizes the gaussian approximation to the energy functional is smaller than the trap oscillator parameter $b$ for $a<0$ and larger than $b$ for $a>0$, consistently with the attractive and repulsive character of the mean field.

These features are not just artifacts of the gaussian ansatz, but are preserved in unconstrained studies[14] of the energy functional. Calculated values e.g. for the critical number $N_{c}$ associated with the loss of the secondary minimum in the case of attractive two-body interactions are consistent with experimentally measured condensate populations.

Assuming the effective two-body potential to have repulsive character, i.e., for $\lambda>0$, it has been proven[15] that the energy functional (??) has an absolute minimum which defines a single-particle wavefunction $\varphi_{0}(\vec{r})$ up to an overall phase factor, which can always be chosen so that $\varphi_{0}(\vec{r})$ is strictly positive. This wavefunction is a solution of a nonlinear, Hartree-like equation obtained by variation of the energy functional with respect to $\varphi(\vec{r})$, the normalization constraint being taken care of in terms of a Lagrange multiplier $\eta$. It reads

$$
\left(-\frac{\hbar^{2} \nabla^{2}}{2 M}+V(\vec{r})\right) \varphi(\vec{r})+\lambda(N-1)|\varphi(\vec{r})|^{2} \varphi(\vec{r})=\frac{\eta}{N} \varphi(\vec{r})
$$

and is known as the Gross-Pitaevski equation. Accordingly, the energy functional (??) has been called the GrossPitaevski functional, and the many-body state $|\Phi\rangle$ constructed from its minimizer (for repulsive effective interaction) the Gross-Pitaevski ground-state. The Lagrange multiplier $\eta$ is seen to be a Hartree-like single particle energy. Its relation to the value of the functional for the corresponding single-particle wavefunction is easily obtained multiplying the Gross-Pitaevski equation by the (conjugate) wavefunction and integrating over $\vec{r}$. It can be expressed in either of two forms, also familiar from the relation between Hartree single-particle energies and the total Hartree mean energy

$$
\begin{aligned}
\eta & =\mathcal{E}[\varphi(\vec{r})]+\frac{\lambda}{2} N(N-1) \int d^{3} r|\varphi(\vec{r})|^{4}= \\
& =2 \mathcal{E}[\varphi(\vec{r})]-N \int d^{3} r \varphi^{*}(\vec{r})\left(-\frac{\hbar^{2} \nabla^{2}}{2 M}+V(\vec{r})\right) \varphi(\vec{r})
\end{aligned}
$$

The integral appearing in the first of these two forms is in fact an integral of the squared single-particle density, which can therefore be interpreted as the average singleparticle density associated with the single-particle wavefunction $\varphi(\vec{r})$. Furthermore, if the factor $N(N-1)$ is replaced by $N^{2}$ in the Gross-Pitaevski functional, and the factor $(N-1)$ is consistently replaced by $N$ in the GrossPitaevski equation (2.11) (an essentially innocuous replacement for large $N$ ), one can write

$$
\frac{\eta}{N}=\frac{\partial \mathcal{E}[\varphi(\vec{r})]}{\partial N} \equiv \mu
$$

which accounts for the fact that this quantity is interpreted as the chemical potential.

It should be stressed at this point that this "mean field" treatment differs from an ordinary Hartree approximation in that the effective two-body interaction is related to the twobody scattering amplitude rather that to the two-body po- tential, which is actually involved to all orders in $v_{\text {eff }}$. Thus it should be rather understood as an approximation of the Brueckner-Hartree type, widely used in the context of nuclear physics[16], further restricted by the scattering length limit used to obtain the effective two-body interaction. Thus, considerable care and judgment are indispensable when using the effective hamiltonian (2.4) in dynamical situations. In particular, the physics involved in the fate of the "collapsing" condensate with attractive effective interactions lies entirely beyond the most optimistically drawn theoretical horizon of this effective hamiltonian, even though the observed meta-stability and its limits may still be meaningfully assessed. Also beyond this horizon is the physics of threebody recombination processes, and therefore also the instability of the Gross-Pitaevski ground state under such processes. 


\subsubsection{The uniform gas and the "healing length"}

Here and in the following subsection we consider some properties of solutions in the case of a repulsive effective two-body approximation $(a>0)$. The energy of the GrossPitaevski ground state is given by the minimum value of the energy functional, which can be expressed using relations (2.12) as

$$
\begin{aligned}
\mathcal{E}\left[\varphi_{0}(\vec{r})\right] & =\eta_{0}-\frac{\lambda}{2} N(N-1) \int d^{3} r\left|\varphi_{0}(\vec{r})\right|^{4}= \\
& =\frac{1}{2}\left[\eta_{0}+N \int d^{3} r \varphi_{0}^{*}(\vec{r})\left(-\frac{\hbar^{2} \nabla^{2}}{2 M}+V(\vec{r})\right) \varphi_{0}(\vec{r})\right]
\end{aligned}
$$

where $\eta_{0} / N$ is the chemical potential associated with the minimizer function $\varphi_{0}(\vec{r})$. To obtain this energy one has in general to solve the Gross-Pitaevsli equation, now a standard numerical task[14].

In the special case of a uniform gas (for which the potential $V(\vec{r})$ representing the trap vanishes identically) translational invariance requires the solutions to be plane waves, so that the ground state energy can be evaluated analytically. Using periodical boundary conditions in a volume $L^{3}$, eq. (2.11) gives for a plane wave of momentum $\vec{k}$ normalized in the quantization volume

$$
\frac{\hbar^{2} k^{2}}{2 M}+\lambda \frac{(N-1)}{L^{3}}=\frac{\eta}{N},
$$

where $N$ is the number of bosons in volume $L^{3}$. Now the second form of the expression (2.13) for the energy shows that the minimizer in this case is the plane wave with zero momentum. Note that this satisfies the stated general properties of the minimizer. The ground state energy is therefore

$$
\mathcal{E}_{0}=\frac{1}{2} \lambda \frac{N(N-1)}{L^{3}}=N \times 4 \pi \frac{\hbar^{2}}{2 M} \rho_{P} a .
$$

In the last step the expression of $\lambda$ in terms of the scattering length has been used, together with the replacement $(N-1) / L^{3} \rightarrow N / L^{3}=\rho_{P}$, here fully allowed by the thermodinamic limit.

An interesting feature of this result is that it reveals a new length parameter which is relevant for the non-ideal gas, given by

$$
\xi_{H} \equiv \frac{1}{\sqrt{4 \pi \rho_{P} a}},
$$

in terms of which the ground state energy per particle of the uniform gas is given as $\mathcal{E}_{0} / N=\hbar^{2} / 2 M \xi_{H}^{2}$. It is referred to as the healing length, or also, alternatively, as the de Broglie wavelength (which however should not be confused with the thermal de Broglie wavelength $\lambda_{d B}$ introduced earlier). In order to understand its meaning, consider the GrossPitaevski ground state in semi-infinite space $x>0$, with an infinite potential wall at $x=0$. This requires that the wavefunction vanishes for $x=0$, being otherwise a solution of

$$
\begin{gathered}
-\frac{\hbar^{2}}{2 M} \nabla^{2} \varphi_{0}(\vec{r})+\lambda\left|\varphi_{0}(\vec{r})\right|^{2} \varphi_{0}(\vec{r})=\mu \varphi_{0}(\vec{r}), \\
\vec{r} \equiv\{x, y, z\}, \quad x>0 .
\end{gathered}
$$

The factor $(N-1)$ has been absorbed in the normalization of the wavefunction by requiring that

$$
\left|\varphi_{0}(\vec{r})\right|^{2} \stackrel{x \rightarrow \infty}{\longrightarrow} \rho_{P} .
$$

The relevant solution to this equation can be found by using the ansatz

$$
\varphi_{0}(\vec{r})=\sqrt{\rho_{P}} \tanh \kappa x, \quad x>0,
$$

independent of $y$ and $z$, which satisfies the required boundary condition at $x=0$ as well as the prescribed normalization condition. In fact, substitution into the equation shows that it is satisfied provided $\kappa$ is chosen so that

or

$$
\kappa^{2}=\lambda \frac{M}{\hbar^{2}} \rho_{P}
$$

$$
\kappa=\sqrt{\frac{\lambda M \rho_{P}}{\hbar^{2}}}=\sqrt{4 \pi \rho_{P} a}=\frac{1}{\xi_{H}}
$$

with $\mu=\hbar^{2} \kappa^{2} / M$. The length $\xi_{H}$ therefore characterizes the scale at which the effect of the potential wall "heals", as one moves away from it and the density approaches its asymptotic value.

There are therefore three distinct scales of length which are relevant in the case of the (uniform) gas, namely the interaction length characterized by $a$, the mean interparticle distance characterized by $\rho_{P}^{-1 / 3}$ and the healing length, characterized by $\left(\rho_{P} a\right)^{-1 / 2}$. For a dilute gas one has $\rho_{P} a^{3} \ll 1$, so that $a \ll \rho_{P}^{-1 / 3}$. Diluteness also implies, moreover, that

$$
\frac{\rho_{P}^{-1 / 3}}{\left(\rho_{P} a\right)^{-1 / 2}}=\sqrt{\rho_{p} a^{3}} \ll 1
$$

so that in the limit of a dilute gas one has the hierarchy of length scales

$$
a \ll \rho_{P}^{-1 / 3} \ll \xi_{H} \sim\left(\rho_{P} a\right)^{-1 / 2} .
$$




\subsubsection{The Thomas-Fermi regime}

The values of the parameter $\gamma$, eq. (2.9), which can be currently be achieved in condensates of atoms with positive scattering length can easily be large compared to one. As an example, a condensate of $10^{6}$ atoms of ${ }^{87} \mathrm{Rb}$, for which $a \simeq 100 r_{B}$ ( $r_{B}$ being the Bohr radius), in an harmonic trap with frequency $\nu=100 \mathrm{~Hz}$, one gets $\gamma \simeq 300$. In such cases not only the condensate becomes quite appreciably wider than the gaussian corresponding to the ground state wavefunction of the trap (see fig. (b)), but the contribution of the kinetic energy term to the Gross-Pitaevski functional becomes small in comparison with the sum of the contributions due to the trap potential and to the two-body interaction. In this case a fair approximation consists in dropping the kinetic energy term altogether. As far as the minimizer of the functional is concerned, this approximation amounts to treating the condensate as being "locally uniform" with a position dependent density, and is therefore known as the Thomas-Fermi approximation to the Gross-Pitaevski functional. It reads

$$
\begin{gathered}
\mathcal{E}_{T F}[\varphi(\vec{r})]=N \int d^{3} r \varphi^{*}(\vec{r}) V(\vec{r}) \varphi(\vec{r}) \\
+\frac{\lambda}{2} N^{2} \int d^{3} r|\varphi(\vec{r})|^{4} .
\end{gathered}
$$

Note that the factor $N(N-1)$ has been replaced by $N^{2}$ in the two-body term. This approximation clearly breaks down near the outer edge of the minimizer wavefunction, where the two retained terms become small. However, for large enough values of $\gamma$, kinetic energy corrections to $\varphi(\vec{r})$ are small even there, on the scale of its peak value.

The equation satisfied by the minimizer of the ThomasFermi approximation to the energy functional can again be obtained by variation of $\mathcal{E}_{T F}$ with respect to $\varphi(\vec{r})$ with the appropriate Lagrange multiplier $\mu$ to take the normalization condition into account. One gets (cf. eq. (2.11))

$$
\left(\mu-V(\vec{r})-N \lambda|\varphi(\vec{r})|^{2}\right) \varphi(\vec{r})=0
$$

which is just an algebraic equation that can be readily solved to give

$$
|\varphi(\vec{r})|^{2}=\left\{\begin{array}{cc}
\frac{\mu-V(\vec{r})}{N \lambda} & \text { for } \quad \mu>V(\vec{r}) \\
0 & \text { for } \quad \mu \leq V(\vec{r}) .
\end{array}\right.
$$

Note that this solution has a discontinuous derivative at points $\vec{r}$ such that $\mu=V(\vec{r})$. The effect of the neglected kinetic energy term is essentially to smoothen the minimizer wavefunction in the vicinity of these points[17]. The value of the Lagrange multiplier $\mu$ is determined from the normalization condition

$$
\int d^{3} r|\varphi(\vec{r})|^{2}=1
$$

or

$$
\int_{\mu>V(\vec{r})} d^{3} r \frac{\mu-V(\vec{r})}{\lambda}=N
$$

\subsection{Spin-dependent effective interaction}

Present day traps can be set in ways which do not rely on hyperfine Zeeman displacements for their operation (as is the case with magnetic traps), allowing for the use of atomic spins as active internal dynamical variables of the manyboson gas. Bose-Einstein condensation in such systems results in what is generally called "spinor condensates".

The atomic spin $f$ results from the coupling of the nuclear spin with the electronic spin, both of which have halfinteger values in the case of the alkali atoms. The nuclei of these atoms have odd charge number $Z$, so that the isotopes corresponding to bosonic atoms must have an even number of neutrons and therefore odd mass numbers. Their lowest electronic configuration has spin $\frac{1}{2}$ due to shell effects, and therefore larger values of $f$ occur for the atoms with larger nuclear spin. The only stable cesium isotope has mass number 133 and nuclear spin $\frac{7}{2}$, so that the lowest atomic hyperfine levels have $f=3$ and $f=4 .{ }^{23} \mathrm{Na}$ and ${ }^{87} \mathrm{Rb}$ are spin $\frac{3}{2}$ nuclei, so that they have $f$ values of 1 and 2 , and ${ }^{85} \mathrm{Rb}$ has nuclear spin $\frac{5}{2}$ and $f$ values of 2 and 3 .

The two-body effective interaction between atoms with spin $f \neq 0$ will in general be spin dependent, the spin dependence being however subjected to restrictions based on general symmetry arguments, such as rotational as well as translational invariance. Interactions being restricted to $s$-waves, rotational invariance amounts to the conservation of the total spin of the interacting pair, and the exchange symmetry required by Bose-Einstein statistics restricts the allowed values of the total spin to even values. This restriction follows from the symmetry property of Clebsch-Gordan coefficients coupling two spins $f$ to total spin $S$

$$
\begin{aligned}
& C_{m_{1} m_{2} M}^{f \underset{f}{f} S}=(-1)^{f+f-S} \underset{m_{m_{2}} m_{1} M}{f \underset{S}{f} S}, \\
& M=m_{1}+m_{2}, \quad-f \leq m_{i} \leq f, \quad-S \leq M \leq S .
\end{aligned}
$$

An often used parameterization[19] of the effective, spin dependent two-body interaction consists therefore in generalizing eq. (2.3) by allowing for different scattering lengths for each of the possible (even) values of the total spin $S$. This implies two scattering lengths for $f=1$, three for $f=2$, etc. Numbers for these parameters are experimentally poorly known (if at all) especially for atoms with the higher $f$ values. In regard to simplicity we restrict the following treatment to a spin dependence which is given in terms of just the standard scalar bilinear in the two spin operators, and generalize eq. (2.3) by writing

$$
v_{\text {eff }}\left(\vec{r}_{1}, \vec{F}_{1}, \vec{r}_{2}, \vec{F}_{2}\right)=\left(\lambda_{0}+\frac{\lambda_{s}}{\hbar^{2}} \vec{F}_{1} \cdot \vec{F}_{2}\right) \delta\left(\vec{r}_{1}-\vec{r}_{2}\right)
$$

where $\vec{F}_{i}, i=1,2$ are the spin operators for the interacting bosons and the constants $\lambda_{0}$ and $\lambda_{s}$ account for possibly different scattering lengths for different values of the total spin. This particular form involves only two independent parameters, even though e.g. for $f \geq 2$ it gives $f+1$ different scattering lengths. Of course the relation among them implied by the two-parameter interaction can in principle be checked experimentally. The two independent scattering lengths $a_{0}$ 
and $a_{2}$ which are allowed for $f=1$, corresponding respectively to $S=0$ and $S=2$, can always be accommodated in the form (2.16), which therefore implies no restriction in this case. Their relation to the parameters $\lambda_{0}$ and $\lambda_{s}$ is then

$$
\lambda_{0}=\frac{4 \pi \hbar^{2}\left(a_{0}+2 a_{2}\right)}{3 M}
$$

and

$$
\lambda_{s}=\frac{4 \pi \hbar^{2}\left(a_{2}-a_{0}\right)}{3 M},
$$

so that when $a_{0}=a_{2}$ one recovers a spin independent effective interaction. For $f=1$ states of ${ }^{23} \mathrm{Na}$ one calculates[18] a positive $\lambda_{s}$ which is of the order of $10 \%$ of $\lambda_{0}$, corresponding to a rather weak spin dependence. The value of $\bar{a}=\left(a_{0}+2 a_{2}\right) / 3$ in this case is about 50 Bohr radii.

In oder to handle the spin variable in a second-quantized formulation one considers a spinor set of field operators $\psi_{m}^{\dagger}(\vec{r}), \psi_{m}(\vec{r}),-f \leq m \leq f$ satisfying the commutation relations

$$
\begin{gathered}
{\left[\psi_{m}(\vec{r}), \psi_{m^{\prime}}^{\dagger}\left(\vec{r}^{\prime}\right)\right]=\delta_{m m^{\prime}} \delta\left(\vec{r}-\vec{r}^{\prime}\right),} \\
{\left[\psi_{m}(\vec{r}), \psi_{m^{\prime}}\left(\vec{r}^{\prime}\right)\right]=0 .}
\end{gathered}
$$

What is understood by the qualification "spinor set" is the fact that the $2 f+1$ different $m$-components transform under rotations as a Racah tensor of rank $f$. The resulting secondquantized form for a set of spinor bosons with spin $f$ can therefore be written as a sum of one- and two-body parts as

$$
H_{\mathrm{eff}}=\int d^{3} r\left(\mathcal{H}_{1}(\vec{r})+\mathcal{H}_{2}(\vec{r})\right)
$$

with the hamiltonian densities

$$
\mathcal{H}_{1}(\vec{r})=\sum_{m_{1} m_{1}^{\prime}} \psi_{m_{1}}^{\dagger}(\vec{r})\left[-\frac{\hbar^{2} \nabla^{2}}{2 M} \delta_{m_{1} m_{1}^{\prime}}+V_{m_{1} m_{1}^{\prime}}(\vec{r})\right] \psi_{m_{1}^{\prime}}(\vec{r})
$$

and

$$
\mathcal{H}_{2}(\vec{r})=\sum_{m_{1} m_{1}^{\prime} m_{2} m_{2}^{\prime}}\left[\frac{\lambda_{0}}{2} \delta_{m_{1} m_{1}^{\prime}} \delta_{m_{2} m_{2}^{\prime}}+\frac{\lambda_{s}}{2 \hbar^{2}} \vec{F}_{m_{1} m_{1}^{\prime}} \cdot \vec{F}_{m_{2} m_{2}^{\prime}}\right] \psi_{m_{1}}^{\dagger}(\vec{r}) \psi_{m_{2}}^{\dagger}(\vec{r}) \psi_{m_{1}^{\prime}}(\vec{r}) \psi_{m_{2}^{\prime}}(\vec{r})
$$

In the one-body part, the trap potential $V_{m m^{\prime}}(\vec{r})$ is, in the simplest case, not only spin-diagonal but also spin independent, $V(\vec{r}) \delta_{m m^{\prime}}$. As written, it may include as well an arbitrary (but time-independent) external magnetic field. In the two-body part, the matrix elements of the 1-particle spin operators, $\vec{F}_{m m^{\prime}}$ can be written quite generally (i.e., for arbitrary spin $f$ ) in terms of Clebsch-Gordan coefficients and of a reduced matrix element. In terms of the spherical components

$$
F_{0} \equiv F_{z}, \quad F_{ \pm 1} \equiv \mp \frac{F_{x} \pm i F_{y}}{\sqrt{2}}
$$

one has, by the Wigner-Eckart theorem

$$
\left(F_{\sigma}\right)_{m m^{\prime}} \equiv\left\langle f m\left|F_{\sigma}\right| f m^{\prime}\right\rangle=\frac{C_{m^{\prime} \sigma m}^{f} \frac{f}{\sqrt{2 f+1}}}{\sqrt{2 f}}\langle f\|F\| f\rangle
$$

where the $C$ symbol is a Clebsch-Gordan coefficient and last factor is the reduced matrix element. It can be calculated from the same formula and the choice of making $F_{z}$ diagonal, with eigenvalues $\hbar m$. Thus, for $f=1$, $\langle 1\|F\| 1\rangle=\sqrt{6} \hbar$.

\subsubsection{Gross-Pitaevski and Thomas-Fermi treatments}

An effective mean-field treatment of spinor condensates can be obtained by a straightforward generalization of what has been done in section 2.2. Again we use an ansatz the normalized $N$-boson state

$$
|N\rangle=\frac{1}{\sqrt{N !}} a^{\dagger N}|0\rangle
$$

where now $a^{\dagger}$ is a spinor creation operator defined as

$$
a^{\dagger} \equiv \sum_{m} \int d^{3} r u_{m}(\vec{r}) \psi_{m}^{\dagger}(\vec{r})
$$

with the normalization condition

$$
\sum_{m} \int d^{3} r\left|u_{m}(\vec{r})\right|^{2}=1 .
$$

This implies that the single-particle wavefunction $\varphi(\vec{r})$ is now a spinor wavefunction with $2 f+1$ components $u_{m}(\vec{r})$, $-f \leq m \leq f$. Since $\psi_{m}(\vec{r})|N\rangle=\sqrt{N} u_{m}(\vec{r})|N-1\rangle$ one obtains for the energy functional 


$$
\begin{aligned}
\mathcal{E}[\varphi(\vec{r})] & \equiv\left\langle N\left|H_{\mathrm{eff}}\right| N\right\rangle=N \sum_{m_{1} m_{1}^{\prime}} \int d^{3} r u_{m_{1}}^{*}(\vec{r})\left(-\frac{\hbar^{2} \nabla^{2}}{2 M} \delta_{m_{1} m_{1}^{\prime}}+V_{m_{1} m_{1}^{\prime}}(\vec{r})\right) u_{m_{1}^{\prime}}(\vec{r})+ \\
& +N(N-1) \frac{\lambda_{0}}{2} \sum_{m_{1} m_{2}} \int d^{3} r\left|u_{m_{1}}(\vec{r})\right|^{2}\left|u_{m_{2}}(\vec{r})\right|^{2}+ \\
& +\frac{N(N-1) \lambda_{s}}{2 \hbar^{2}} \sum_{m_{1} m_{1}^{\prime} m_{2} m_{2}^{\prime}} \int d^{3} r u_{m_{1}}(\vec{r}) u_{m_{2}}(\vec{r}) u_{m_{1}^{\prime}}(\vec{r}) u_{m_{2}^{\prime}}(\vec{r}) \vec{F}_{m_{1} m_{1}^{\prime}} \cdot \vec{F}_{m_{2} m_{2}^{\prime}}
\end{aligned}
$$

The variational condition that determines the spinor component functions $u_{m}(\vec{r})$ is

$$
\delta\left(\left\langle N\left|H_{\mathrm{eff}}\right| N\right\rangle-\eta \sum_{m}\left\langle u_{m} \mid u_{m}\right\rangle\right)=0,
$$

variations being taken with respect to the $u_{m}(\vec{r})$. The term involving the Lagrange multiplier $\eta$ accounts for the normalization condition on the spinor state. This leads to the coupled nonlinear equations

$$
\begin{aligned}
-\frac{\hbar^{2} \nabla^{2}}{2 M} u_{m}(\vec{r}) & +\sum_{m^{\prime}} V_{m m^{\prime}}(\vec{r}) u_{m^{\prime}}(\vec{r})+(N-1) \lambda_{0} \sum_{m^{\prime}}\left|u_{m^{\prime}}(\vec{r})\right|^{2} u_{m}(\vec{r})+ \\
& +\frac{(N-1) \lambda_{s}}{\hbar^{2}} \sum_{m^{\prime} m_{1} m_{1}^{\prime}} \vec{F}_{m m^{\prime}} \cdot \vec{F}_{m_{1} m_{1}^{\prime}} u_{m_{1}}^{*}(\vec{r}) u_{m_{1}^{\prime}}(\vec{r}) u_{m^{\prime}}(\vec{r})=\frac{\eta}{N} u_{m}(\vec{r}) .
\end{aligned}
$$

The factors $N-1$ are usually replaced by $N$. In the ThomasFermi limit, the kinetic energy term is negligible and the determination of the spinor component functions is again pointwise algebraic. Recall that the trap potential $V_{m m^{\prime}}(\vec{r})$ is, in the simplest case, not only spin-diagonal but also spin independent; as written, it may include as well an arbitrary (but time-independent) external magnetic field. The spin dependent term involves the operator

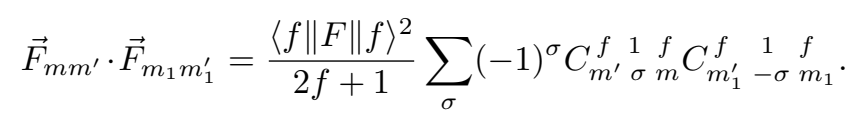

In fact there is no sum over $\sigma$, since its value is determined by the consistent values of the remaining $m$ 's.

As an example, we write down explicitly the coupled
Thomas-Fermi equations for $f=1$ bosons in a spindiagonal trap potential $V_{m m^{\prime}}(\vec{r}) \rightarrow V_{m}(\vec{r}) \delta_{m m^{\prime}}$. The $m$ dependence of the potential may be used, in particular, to account for the first order Zeeman splitting due to a uniform external magnetic field, i.e. $V_{m}(\vec{r})=V_{0}(\vec{r})+m \Delta_{B}$. We get

$$
\vec{F}_{m m^{\prime}} \cdot \vec{F}_{m_{1} m_{1}^{\prime}}=2 \hbar^{2}(-1)^{\sigma} C_{m^{\prime} \sigma m}^{11} C_{m_{1}^{\prime}}^{1} \underset{-\sigma m_{1}}{1}
$$

with $\sigma=m-m^{\prime}=m_{1}^{\prime}-m_{1}$, which includes a condition on the possible sets of $m$-values that give nonzero contribution. Ignoring the kinetic energy term then gives the coupled Thomas-Fermi equations for the spinor component wavefunctions $u_{m}(\vec{r}), m=0, \pm 1$

$$
\begin{aligned}
& {\left[\frac{\eta}{N}-V_{1}(\vec{r})-(N-1)\left(\lambda_{0}+\lambda_{s}\right) \rho_{T}(\vec{r})\right] u_{1}(\vec{r})=(N-1) \lambda_{s}\left[u_{-1}^{*}(\vec{r}) u_{0}^{2}(\vec{r})-2\left|u_{-1}(\vec{r})\right|^{2} u_{1}(\vec{r})\right]} \\
& {\left[\frac{\eta}{N}-V_{0}(\vec{r})-(N-1)\left(\lambda_{0}+\lambda_{s}\right) \rho_{T}(\vec{r})\right] u_{0}(\vec{r})=(N-1) \lambda_{s}\left[2 u_{0}^{*}(\vec{r}) u_{1}(\vec{r}) u_{-1}(\vec{r})-\left|u_{0}(\vec{r})\right|^{2} u_{0}(\vec{r})\right]} \\
& {\left[\frac{\eta}{N}-V_{-1}(\vec{r})-(N-1)\left(\lambda_{0}+\lambda_{s}\right) \rho_{T}(\vec{r})\right] u_{-1}(\vec{r})=(N-1) \lambda_{s}\left[u_{1}^{*}(\vec{r}) u_{0}^{2}(\vec{r})-2\left|u_{1}(\vec{r})\right|^{2} u_{-1}(\vec{r})\right]}
\end{aligned}
$$


where $^{4} \rho_{T}(\vec{r}) \equiv \sum_{m}\left|u_{m}(\vec{r})\right|^{2}$.

Assuming that the $u_{m}(\vec{r})$ are real except for an overall phase, i.e.,

$$
u_{m}(\vec{r})=\left|u_{m}(\vec{r})\right| e^{i \varphi_{m}},
$$

the equations become real except for a phase $\pm\left(2 \varphi_{0}-\varphi_{1}-\right.$ $\left.\varphi_{-1}\right)$ multiplying the first terms on the right-hand side. In this way, the equations become real if this phase is 0 or $\pi$. In general, this indicates that this approximation is sensitive to phase relations among the spinor components. Further understanding of the phases results from consideration of the Thomas-Fermi approximation to the energy functional per particle (2.20), which is sensitive to the same combination of phases. It is given by

$$
\begin{aligned}
\mathcal{E}_{T F}[\varphi(\vec{r})]= & N \sum_{m} \int d^{3} r u_{m}^{*}(\vec{r}) V_{m}(\vec{r}) u_{m}(\vec{r})+\frac{N(N-1) \lambda_{0}}{2} \sum_{m m^{\prime}} \int d^{3} r\left|u_{m}(\vec{r})\right|^{2}\left|u_{m^{\prime}}(\vec{r})\right|^{2}+ \\
& +\frac{N(N-1) \lambda_{s}}{2 \hbar^{2}} \sum_{m_{1} m_{1}^{\prime} m_{2} m_{2}^{\prime}} \int d^{3} r u_{m_{1}^{\prime}}^{*}(\vec{r}) u_{m_{2}^{\prime}}^{*}(\vec{r}) u_{m_{1}}(\vec{r}) u_{m_{2}}(\vec{r}) \vec{F}_{m_{1} m_{1}^{\prime}} \cdot \vec{F}_{m_{2} m_{2}^{\prime}} .
\end{aligned}
$$

The spin-dependent term is, writing explicitly the weights resulting from the spin matrix elements,

$$
N(N-1) \lambda_{s} \int d^{3} r\left[\frac{1}{2}\left(\left|u_{1}\right|^{4}+\left|u_{-1}\right|^{4}\right)+\left|u_{0}\right|^{2}\left(\left|u_{1}\right|^{2}+\left|u_{-1}\right|^{2}\right)-\left|u_{1}\right|^{2}\left|u_{-1}\right|^{2}+2 \operatorname{Re}\left(u_{0}^{* 2} u_{1} u_{-1}\right)\right],
$$

the last term of which becomes, under the assumptions made above concerning the phases,

$$
2 \operatorname{Re}\left(u_{0}^{* 2} u_{1} u_{-1}\right) \rightarrow 2 \cos \left(\varphi_{1}+\varphi_{-1}-2 \varphi_{0}\right)\left|u_{0}\right|^{2}\left|u_{1}\right|\left|u_{-1}\right|
$$

This gives in fact an explicit expression for the ThomasFermi approximation to the energy functional under the somewhat more restrictive ansatz in which the $u_{m}(\vec{r})$ are real except for an $\vec{r}$-independent phase, therefore only allowing for $\vec{r}$-independent phase relations among the spinor components. At points where neither of the amplitudes $u_{m}(\vec{r})$ is zero, the functional is stationary with respect to phase variations at

$$
2 \varphi_{0}-\varphi_{1}-\varphi_{-1}=0
$$

or

$$
2 \varphi_{0}-\varphi_{1}-\varphi_{-1}=\pi
$$

The occurrence of this particular combination of the three phases results from conservation of the third component of the total spin of two interacting bosons, which allows for converting one $m_{1}=m_{2}=0$ pair into a $m_{1}=1, m_{2}=$ -1 pair and vice-versa. Variation with respect to the (absolute values of the) amplitudes yields the Thomas-Fermi equations as written before, with the adopted choice for the phase $\left(2 \varphi_{0}-\varphi_{1}-\varphi_{-1}\right)$.

Thomas-Fermi solutions for spinor condensates are much richer than what one obtains in the case of a single component. One of the simplest examples (albeit involving parameter values which are rather unrealistic in experimental terms) is shown in Fig. 2.2. Actually two mutually complementary solutions are shown in this figure. In the first

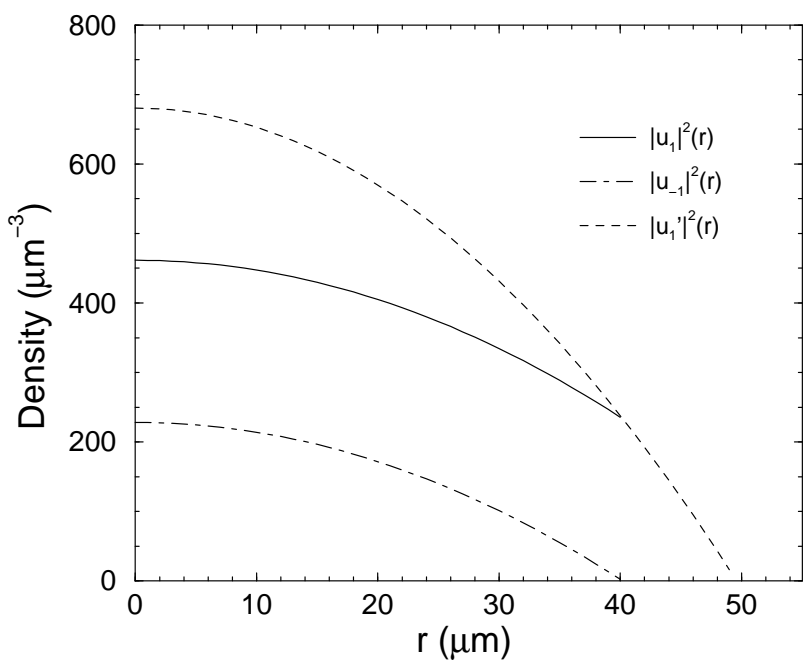

Figure 2.2. Solutions of the T-F equations for sodium atoms in a spherical harmonic trap with frequency $60 \mathrm{~Hz}, \mu=10^{4} \mathrm{~Hz}$ and $\Delta_{B}=-70 \mathrm{~Hz}$. Note that this last value corresponds to an experimentally unrealistic extremely weak magnetic field, of the order of $10^{-4} \mathrm{G}$. The two-body interaction parameters used were $\lambda_{0}=14.5 \mathrm{~Hz} \mu \mathrm{m}^{3}\left(\bar{a} \simeq 50 a_{B}\right)$ and $\lambda_{S}=0.3 \mathrm{~Hz} \mu \mathrm{m}^{3}$ $\left(a_{2}-a_{0} \simeq 3 a_{B}\right)$.

\footnotetext{
${ }^{4}$ Strictly speaking one should take into account the fact that when the one-body part of the hamiltonian is diagonal in the spin projections the quantity $\hat{N}_{1}-\hat{N}_{-1}$ is also conserved, requiring a second Lagrange multiplier $\Lambda$. The effect of this is to replace $\eta / N$ respectively by $\eta / N+\Lambda, \eta / N$ and $\eta / N-\Lambda$ in the three coupled equations above. When the $m$-dependence of the trap potential comes from an overall first order Zeeman shift $\Delta_{B}$, this is in turn equivalent to a redefinition of the Zeeman shift $\Delta_{B} \rightarrow \Delta_{B}^{\prime}=\Delta_{B}+\Lambda$. The solutions obtained without explicitly introducing $\Lambda$ and interpreting $\Delta_{B}$ as the actual first order Zeeman shift correspond therefore to the value of the second conserved quantity for which $\Lambda=0$.
} 
one, the $m=0$ density vanishes identically, and a Zeeman induced relative enhancement of the $m=1$ density increases towards the surface to the point where the $m=-1$ density vanishes. At this point the $m=1$ density joins a different solution which has $u_{0}=u_{-1} \equiv 0$, shown as the dashed line. This latter solution is unstable (in the sense that it corresponds to a maximum of the Thomas-Fermi energy functional) while $u_{-1}(r) \neq 0$ (for the first solution), but becomes stable (in the sense that it becomes associated to a minimum of the Thomas-Fermi energy functional) in the outer skin, so that it may be seen as the extension of the corresponding component of the first solution for larger values of $r$.

It should be noted that the phase-carrying quantity $2 \operatorname{Re}\left(u_{0}^{* 2} u_{1} u_{-1}\right)$ vanishes identically, so that the overall relative phase of the two nonvanishing components of the spinor is not determined. This plain computational fact must be understood as a warning against too stringent limitations imposed by the adopted ansatz (2.18) and (2.19), which prevent physics intended by the effective hamiltonian to be cap- tured by the effective mean-field approximation as implemented.

In order to justify this statement, consider again the spindependent effective hamiltonian (2.17) for the case $f=1$ with the trap potential reduced to a position independent first order Zeeman shift, i.e.

$$
V_{m m^{\prime}}(\vec{r}) \longrightarrow m \Delta_{B} \delta_{m m^{\prime}}
$$

Corresponding to the neglect of the kinetic energy term in the Thomas-Fermi approximation, introduce the momentum state operators (using periodic boundary conditions in a quantization volume $\mathcal{V}$ )

$$
a_{m}(\vec{k}) \equiv \frac{1}{\mathcal{V}} \int_{\mathcal{V}} d^{3} r e^{i \vec{k} \cdot \vec{r}} \psi_{m}(\vec{r}), \quad-1 \leq m \leq 1
$$

and restrict the hamiltonian to the zero-momentum plane waves. The resulting truncated version of the effective hamiltonian is then (cf. [20, 21])

$$
\begin{aligned}
H_{\mathrm{eff}} \rightarrow H_{0}= & \Delta_{B} \sum_{m} m a_{m}^{\dagger} a_{m}+\frac{\lambda_{0}}{2 \mathcal{V}} \sum_{m m^{\prime}} a_{m}^{\dagger} a_{m^{\prime}}^{\dagger} a_{m^{\prime}} a_{m}+ \\
& +\frac{\lambda_{s}}{2 \mathcal{V}}\left(a_{1}^{\dagger} a_{1}^{\dagger} a_{1} a_{1}+a_{-1}^{\dagger} a_{-1}^{\dagger} a_{-1} a_{-1}+2 a_{1}^{\dagger} a_{0}^{\dagger} a_{0} a_{1}+2 a_{-1}^{\dagger} a_{0}^{\dagger} a_{0} a_{-1}-\right. \\
& \left.\quad-2 a_{-1}^{\dagger} a_{1}^{\dagger} a_{1} a_{-1}+2 a_{0}^{\dagger} a_{0}^{\dagger} a_{1} a_{-1}+2 a_{1}^{\dagger} a_{-1}^{\dagger} a_{0} a_{0}\right)= \\
= & \Delta_{B} \hat{S}_{z}+\frac{\lambda_{0}}{2 \mathcal{V}} \hat{N}(\hat{N}-1)+\frac{\lambda_{s}}{2 \mathcal{V}}\left(\vec{S}^{2}-2 \hat{N}\right)
\end{aligned}
$$

where the $a_{m}, a_{m}^{\dagger}$ are now zero momentum operators, $\hat{N}_{m} \equiv a_{m}^{\dagger} a_{m}, \hat{N} \equiv \sum_{m} \hat{N}_{m}$ are number operators and $\vec{S} \equiv\left\{\hat{S}_{x}, \hat{S}_{y}, \hat{S}_{z}\right\}$ is the total spin operator the components of which, for $f=1$, can be written as

$$
\begin{gathered}
\hat{S}_{x}+i \hat{S}_{y}=\sqrt{2}\left(a_{0}^{\dagger} a_{1}+a_{-1}^{\dagger} a_{0}\right), \\
\hat{S}_{x}-i \hat{S}_{y}=\sqrt{2}\left(a_{1}^{\dagger} a_{0}+a_{0}^{\dagger} a_{-1}\right), \\
\hat{S}_{z}=\left(a_{-1}^{\dagger} a_{-1}-a_{1}^{\dagger} a_{1}\right) .
\end{gathered}
$$

Contact with the preceding Thomas-Fermi treatment can be made by noting that the integrand of the Thomas-Fermi energy functional (2.22) can be obtained from this hamiltonian (up to replacement of factors $N(N-1)$ by $N^{2}$ ) using the spinor ansatz

$$
|\Phi\rangle \equiv\left(\begin{array}{c}
\left|\nu_{1}\right\rangle \\
\left|\nu_{0}\right\rangle \\
\left|\nu_{-1}\right\rangle
\end{array}\right), \quad \sum_{m=-1}^{1}\left|\nu_{m}\right|^{2}=N
$$

where the $\left|\nu_{m}\right\rangle$ are coherent states[1] for the operators $a_{m}$, i.e.

$$
\left|\nu_{m}\right\rangle \equiv e^{\nu_{m} a_{m}^{\dagger}-\nu_{m}^{*} a_{m}}|0\rangle
$$

$$
a_{m}\left|\nu_{m}\right\rangle=\nu_{m}\left|\nu_{m}\right\rangle=\left|\nu_{m}\right| e^{i \varphi_{m}}\left|\nu_{m}\right\rangle
$$

where $|0\rangle$ stands for the vacuum state, $a_{m}|0\rangle=0$. A variational treatment therefore leads again to the Thomas-Fermi coupled equations, now written in terms of the coherent amplitudes $\nu_{m}$. The possible solution having $\nu_{0}=0$ leaves the relative phase of the remaining two amplitudes undefined.

One can now however confront the approximation involving this ansatz with the exact ground state of the truncated hamiltonian (2.23) by taking advantage of its expression in terms of number and total spin operators. To see what this ground state is, note that $\hat{N}, \vec{S}^{2}$ and $\hat{S}_{z}$ are commuting operators, so that the simultaneous eigenvectors $|N S M\rangle$ of these three operators are eigenstates of $H_{0}$. The angular momentum commutation relations for the components of the total spin operator give as usual

$$
\begin{gathered}
\vec{S}^{2}|N S M\rangle=S(S+1)|N S M\rangle, \\
\hat{S}_{z}|N S M\rangle=M|N S M\rangle, \quad-S \leq M \leq S ;
\end{gathered}
$$

moreover $S \leq M \leq N$ so that the maximum value of the total spin is $S=N$. The eigenvalues of $H_{0}$ are then 


$$
H_{0}|N S M\rangle=\left(\Delta_{B} M+\frac{\lambda_{0}}{\mathcal{V}} N(N-1)+\frac{\lambda_{s}}{\mathcal{V}}[S(S+1)-2 N]\right)|N S M\rangle
$$

The ground state corresponds to available values of $S$ and $M$ which minimize the eigenvalue of $H_{0}$. When $\lambda_{S}>0$ (as in the example of fig. ) a reduction of $S$ with respect to its maximum value will be favored if $\Delta_{B}$ is not too large.

Except for the simplest "stretched" angular momentum states with $S=N, M= \pm N$ the states $|N S M\rangle$ involve spin correlations that cannot be properly represented in terms of the spinor ansatz. They can in fact be written explicitly in terms of the operators $a_{m}^{\dagger}$. When $M=-S$ they are given by

$|N S M=-S\rangle=\mathcal{N}_{N S} a_{-1}^{\dagger S}\left(a_{0}^{\dagger 2}-2 a_{1}^{\dagger} a_{-1}^{\dagger}\right)^{(N-S) / 2}|0\rangle$

where the correlations are clearly visible in the operator within brackets, which creates a pair of bosons coupled to zero total spin. The normalization factor $\mathcal{N}_{N S}$ is[21]

$\mathcal{N}_{N S}=\left[2^{(N-S) / 2}\left(\frac{N-S}{2}\right) ! S ! \frac{(N+S+1) ! !}{(2 S+1) ! !}\right]^{-1 / 2}$.

States with other values of $M$ can of course be obtained from these by using the total spin projection raising operator $\hat{S}_{+} \equiv \hat{S}_{x}+i \hat{S}_{y}$.

The difficulty found in the guise of an undetermined relative phase when using the simple spinor mean-field ansatz in connection with the spin dependence of the effective twobody interaction reflects therefore the inability of this ansatz to capture essential spin correlations. It should be noted, on the other hand, that the "exact" treatment reviewed here to pinpoint this fact ignored the possible concomitant role of the position degrees of freedom, which are crucial when considering a trapped system of bosons. A better treatment for this type of system should therefore be able to handle both of these aspects dynamically at the same time. Treatments such as that of refs. [20], which merely replace the zero-momentum plane wave used here by one however judiciously chosen frozen single particle wavefunction of course fall short of this aim.

\subsection{Gross-Pitaevski limit}

The understanding of the success of the Gross-Pitaevski (GP) treatment of trapped, dilute atomic condensates has acquired a new facet since the discovery was announced late in 2001, by Lieb and Seiringer[22] (see also[23]), of important results concerning the ground state of a dilute boson gas with repulsive two-body interactions in a rather peculiar limit to which the accumulated G-P successes are in some sense close. This limit, to be called the Gross-Pitaevski limit for reasons that will become clear shortly, differs from the usual thermodynamic limit, which consists in letting the volume go to infinity at constant density. Thus, if the system under consideration is constituted of a given species of bosonic atoms, associated with a scattering length $a>0$, the thermodynamic limit implies its characterization in terms of a given value of the quantity $\rho_{P} a^{3}$. The uniform GrossPitaevski ground state of this system is the minimizer of the G-P functional, which satisfies the equation

$$
-\frac{\hbar^{2}}{2 M} \nabla^{2} \varphi(\vec{r})+\frac{4 \pi \hbar^{2} a}{M}|\varphi(\vec{r})|^{2} \varphi(\vec{r})=\mu \varphi(\vec{r})
$$

with periodic boundary conditions in volume $\mathcal{V}$, the function $\varphi(\vec{r})$ being normalized according to

$$
\int_{\mathcal{V}} d^{3} r|\varphi(\vec{r})|^{2}=N, \quad \frac{N}{\mathcal{V}}=\rho .
$$

The minimizer in this case is just the constant (zero momentum) solution with particle density $\rho_{P}$. It is given explicitly by

$$
|\varphi|^{2}=\frac{\mu M}{4 \pi \hbar^{2} a}=\frac{N}{\mathcal{V}} \quad \text { or } \quad \mu=\frac{4 \pi \hbar^{2} N a}{M \mathcal{V}} .
$$

When one thinks in terms of the thermodynamic limit, this gives the proportionality of the G-P chemical potential $\mu$ on the system density through the effective contact interaction involving the scattering length $a$ (the energy per particle is in this case just $\mu / 2$ ). On the other hand, it also shows that the constant G-P solution remains invariant under a scaling quite unrelated to that which is associated to the thermodynamic limit, namely, when the density and the scattering length are changed in such a way that the product $N a$, and also the quantization volume $\mathcal{V}$, remain constant. It is easy to see that the G-P functional itself remains unchanged under this type of scaling. The Gross-Pitaevski limit consists in letting $N \rightarrow \infty$ with $N a$ constant, so that the scattering length $a \rightarrow 0$ as $N^{-1}$ and $\rho a^{3} \rightarrow 0$ as $N^{-2}$. This limit corresponds therefore to an extremely dilute (even though the particle density diverges linearly with $N$ ) and yet non trivial limit, even though the strength of the effective interaction goes to zero.

The special relevance of this particular limit comes from the proof given by Lieb and Seiringer that, when it is taken, the above G-P ground-state solution becomes exact, i.e., the exact ground state of the full many-body problem. The two-body interaction is given as a two-body potential having suitable properties, besides giving the correct scattering length $a>0$, a sufficient condition for the proof being that it is everywhere positive (e.g. a pure hard core potential with core radius $a$ ). This is of course "unrealistic", but real condensates are in fact systems not in their ground states, but in meta-stable states which are however rendered stable by the unrealistic assumption, and supposedly without 
important collateral effects. The fact that the G-P solution is exact implies, in particular, that the exact ground state is of the simple product form (2.6). This means that the G$\mathrm{P}$ limit eliminates all many-particle correlations induced by the two-particle interaction from the wavefunction. In this sense, the G-P limit is "many-body trivial", even though it differs from an ideal gas in several (one-body) ways (e.g. in the energy per particle). Another side effect of the proof is that it provides for a justification of the adopted form of the two-body effective interaction used in connection with the G-P functional at least for purely repulsive two-body potentials. Note that for this type of potential the scattering length is always positive.

It is important to stress here that the result of Lieb and Seiringer depends in an essential way on the G-P functional not corresponding to a plain mean field approximation of the Hartree type, but rather to an effective mean field approximation which at best can be described as being of the Brueckner type, as emphasized earlier. The exactness of the product wavefunction based on the minimizer of the functional does not hold if the effective two-body interaction is replaced by the actual two-body interaction potential.

The scaling property relevant for the Gross-Pitaevski limit holds also for trapped bosons, in which case the equation for the minimizer of the Gross-Pitaevski functional reads

$$
\left[-\frac{\hbar^{2}}{2 M} \nabla^{2}+V(\vec{r})\right] \varphi(\vec{r})+\frac{4 \pi \hbar^{2} a}{M}|\varphi(\vec{r})|^{2} \varphi(\vec{r})=\mu \varphi(\vec{r}) .
$$

Due to the presence of the trapping external potential $V(\vec{r})$

$$
a \ll \rho_{P}^{-\frac{1}{3}} \ll \xi_{H}=\left(\rho_{P} a\right)^{-\frac{1}{2}} \quad \Longrightarrow
$$

\section{Roles for atomic structure}

When discussing the condensation process from a theoretical point of view, the atoms which constitute the gas are treated as identical bosons, possibly endowed with nonzero spin but otherwise "structureless" objects. This picture is of course completely inadequate for practical purposes relating e.g. to the achievement of confinement, cooling and condensation of the bosonic gas, since each of these operations takes advantage of internal atomic degrees of freedom for its implementation in the laboratory. This is of course a rich and challenging field of its own, which we will not consider here, however. The topics included in this chapter refer instead to atomic structure properties in so far as they can be used to control (as it is the case of the "Feshbach resonances") or enrich (as it is the case of "hybrid condensates" involving different atomic species, atoms and molecules, etc.) the dynamics of dilute Bose-Einstein condensates as such, rather than just adding "theoretical realism" to the picture. the relevant solution is normalizable. Since the solution $\varphi_{N, a}(\vec{r})$ for the case of $N$ trapped bosons and scattering length $a$ is also assumed to be normalized according to

$$
\int d^{3} r\left|\varphi_{N, a}(\vec{r})\right|^{2}=N
$$

it changes under scale transformations $N \rightarrow N / \xi, a \rightarrow \xi a$ that leave the product $N a$ fixed according to

$$
\varphi_{N, a}(\vec{r})=\frac{1}{\sqrt{\xi}} \varphi_{\frac{N}{\xi}, a \xi}(\vec{r}) .
$$

In this case the G-P density is of course not uniform, and keeps a fixed "geometry" under G-P scaling. It also becomes exact in the G-P limit, so that in this limit the trapped system ground state is also "many-body trivial", even though not ideal.

The successes of descriptions based on the G-P approximation can be associated with proximity of the relevant experimental parameters to this particular limit, in which important interaction effects survive in the condensate geometry, while correlation effects are in some sense negligibly small (and vanish rigorously in the G-P limit). Typical values for experimentally achieved condensates are $a \sim 10^{-7}$ $\mathrm{cm}, N \sim 10^{5}, \rho_{P} \sim 10^{15} \mathrm{~cm}^{-3}$, which correspond to $\rho_{P} a^{3} \sim 10^{-6}, \rho_{P}^{-1 / 3} \sim 10^{-5} \mathrm{~cm}$ and healing length $\xi_{H}=\left(\rho_{P} a\right)^{-1 / 2} \sim 10^{-4} \mathrm{~cm}$. One has thus typically the characteristic three-lengths hierarchy (2.15) of a dilute system. In the G- P limit ( $a \rightarrow 0$ with $N a$ constant) this hierarchy is strengthened as can be seen through the $N$ dependence of the various lengths:

$$
\mathcal{O}\left(\frac{1}{N}\right) \ll \mathcal{O}\left(\frac{1}{N^{1 / 3}}\right) \ll \mathcal{O}\left(N^{0}\right)
$$

\subsection{Atom-atom resonance scattering and the effective two-body interaction}

Low-energy resonances can play a decisive role in determining the atom-atom scattering length. Since this quantity determines the effective two-body interaction to be used in connection with the Gross-Pitaevski functional, the possibility of tuning the position in energy of narrow low-energy resonances by means of some external control variable (such as an external magnetic field, acting through the Zeeman shifts it produces) may allow for tuning the value of the scattering length, and hence also of the effective two-body interaction. The resonances one is interested in here are not "shape resonances" related to the properties of the atomatom interaction potential, but the typically much narrower and numerous many-body resonances associated to particular quasi-stationary states of the two-atom system. They are therefore related in an essential way to atomic structure 
properties, and can be seen as atomic analogues of the "compound nucleus" resonances of nuclear physics. One general collision theory, which in particular gives a nice account of the effects of quasi stationary states of the compound system on the scattering observables has been developed since the 1960's by Feshbach[24], mostly in the context of nuclear physics. The use of the concepts of this particular theory in the atomic domain has led to the denomination "Feshbach resonances" for the resonances involving internal degrees of freedom of the atom-atom compound system. They are now widely used as a tool for achieving experimental control of the two-body effective interaction in many experiments involving cold, dilute atomic gases of bosons and also of fermions[2, 3, 4, 5, 25].

In the "no Feshbach resonance" case, the determination of the scattering amplitude $a$ involves solving the Schrdinger equation

$$
\left[E-\frac{p^{2}}{2 \mu_{R}}-V\right]\left|\Psi_{\vec{k}}\right\rangle=0
$$

where $\vec{p}$ is the relative momentum and $\mu_{R}$ is the reduced mass of the two colliding atoms. The interaction between the two atoms is described by the potential $V$, and the energy scale is set so that this potential vanishes in the limit of very large distance between them. For simplicity we omit any explicit consideration of spins. The center of mass energy of the colliding system is $E>0$, also equal to the asymptotic value of the kinetic energy of relative motion of the two atoms. If the pre-collision asymptotic relative momentum is $\hbar \vec{k}$ one has therefore

$$
E=\frac{\hbar^{2} k^{2}}{2 \mu_{R}} .
$$

The scattering state vector $\left|\Psi_{\vec{k}}\right\rangle$, or equivalently its associated wavefunction $\left\langle\vec{r} \mid \Psi_{\vec{r}}\right\rangle$, are further determined by the usual scattering asymptotic boundary condition

$$
\left\langle\vec{r} \mid \Psi_{\vec{k}}\right\rangle \quad \stackrel{r \rightarrow \infty}{\longrightarrow} e^{i \vec{k} \cdot \vec{r}}+f(\hat{r}) \frac{e^{i k r}}{r}
$$

where the argument $\hat{r}$ of the scattering amplitude $f$ denotes the angular components of the relative position vector $\vec{r}$. This boundary condition implies the plane-wave-like orthogonality relation expressed in terms of the Dirac delta function as

$$
\left\langle\Psi_{\vec{k}} \mid \Psi_{\overrightarrow{k^{\prime}}}\right\rangle=(2 \pi)^{3} \delta\left(\vec{k}-\overrightarrow{k^{\prime}}\right) .
$$

The scattering length is a particular way to represent the extreme low energy (meaning $E \rightarrow 0$ ) scattering amplitude, which can in general be extracted from the $s$-wave component $\left\langle\vec{r} \mid \psi_{(0) \vec{k}}\right\rangle$ of the scattering wavefunction. The asymptotic boundary condition corresponding to eq. (3.2) to be satisfied by this particular partial wave is

$$
\left\langle\vec{r} \mid \Psi_{\vec{k}}\right\rangle_{(l=0)} \equiv e^{i \delta_{0}(k)} u_{0 k}(r) \quad \stackrel{r \rightarrow \infty}{\longrightarrow}-\frac{1}{2 i k r}\left(e^{-i k r}-e^{2 i \delta_{0}(k)} e^{i k r}\right)=e^{i \delta_{0}} \frac{\sin \left(k r+\delta_{0}\right)}{k r}
$$

which determines the $s$-wave phase shift $\delta_{0}(k)$. The $s$-wave component of the scattering amplitude does not depend on the angular variables and is given by

$$
f_{(l=0)}=\frac{e^{2 i \delta_{0}(k)}-1}{2 i k}=e^{i \delta_{0}(k)} \frac{\sin \delta_{0}}{k} .
$$

The phase factor $e^{2 i \delta_{0}(k)}$ is the zero angular momentum "Smatrix", and the scattering length $a$ is defined as the limit

$$
a=-\lim _{k \rightarrow 0} \frac{\delta_{0}(k)}{k}, \quad \text { so that } \quad \lim _{k \rightarrow 0} f_{(l=0)}=-a \text {, }
$$

and the large distance asymptotic behavior of the $s$-wave amplitude in the limit $k \rightarrow 0$ can be expressed in terms of the scattering length as

$$
e^{i \delta_{0}} u_{0 k}(r) \stackrel{r \rightarrow \infty}{\longrightarrow} e^{i \delta_{0}} \frac{\sin \left(k r+\delta_{0}\right)}{k r} \quad \stackrel{k \rightarrow 0}{\longrightarrow} \quad 1-\frac{a}{r} .
$$

Note furthermore that for very small values of $k$ the zero angular momentum $\mathrm{S}$-matrix can be approximated as

$$
e^{2 i \delta_{0}(k)} \simeq e^{-2 i k a}, \quad k \simeq 0 .
$$

\subsubsection{Effects of internal structure dynamics}

We next modify this simple low-energy elastic scattering picture to introduce the "Feshbach resonance" syndrome[26]. When treating the elastic scattering one assumes a definite and fixed choice for the internal states of the colliding atoms in the asymptotic region. A crucial missing ingredient is, however, the existence of other internal states of higher energy allowing in principle for additional channels, which allow e.g. for inelastic scattering due to internal excitation of one or both atoms. Inelastic scattering will be energetically allowed if the energy $E$ is large enough to leave a positive relative kinetic energy following the excitation process, in which case the corresponding channels are "open channels". At energies $E$ low enough so that all inelastic channels are "closed", one always can in principle describe the scattering on the basis of an equation like (3.1), but then the effects due to the degrees of freedom involved in the closed inelastic channels have to be accounted for by the atom-atom potential $V$, which in general must therefore be a complicated, energy-dependent object containing the full internal dynamics of the two-atom compound system. In order to unravel this situation it is preferable to consider the 
closed channels explicitly, as well as their coupling to the single open, elastic channel. This is essentially what is done in the Feshbach theory.

Although realistic descriptions of the low-energy scattering of two bound many-body systems such as two atoms can be extremely involved, some important and characteristic aspects can be captured even in grossly simplified schematic models. Let us then consider, in addition to the lowest energy internal asymptotic states $|0\rangle$ associated with the open elastic channel, one single excited state $|\epsilon\rangle$ of either atom, with excitation energy $\epsilon$ as measured from the ground state. We will now generalize the scattering problem (3.1) to deal with the scattering of such a pair of two-level atoms. The hamiltonian governing the dynamics of the two-atom system is taken to be

$$
H=\frac{p^{2}}{2 \mu_{R}} \otimes \mathbf{1}+V_{00} \otimes|00\rangle\left\langle 00\left|+\left(V_{0 \epsilon}+\epsilon\right) \otimes\right| 0 \epsilon\right\rangle\langle 0 \epsilon|+v_{c} \otimes(|00\rangle\langle 0 \epsilon|+| 0 \epsilon\rangle\langle 00|) .
$$

Note that each term has two factors, the first acting in the position coordinates of the atoms and the second acting on the space of internal states. The vectors $|00\rangle$ and $|0 \epsilon\rangle$ correspond to respectively to the two atoms being in the ground state $|0\rangle$ and to one atom being in the ground state, the other being in the excited state $|\epsilon\rangle$. Thus $V_{00}$ and $V_{0 \epsilon}$ describe the interaction of the two atoms in each of these two channels, while $v_{c}$ is an additional interaction which couples the two channels, i.e., is able to change the internal state of one of the two atoms. The term involving $\epsilon$ as first factor accounts for the energy of the internal state $|0 \epsilon\rangle$ being higher than that of $|00\rangle$.

The state-vectors of the two atom system described by this hamiltonian will, correspondingly, be of the form

$$
|\Psi\rangle=\left|\psi_{00}\right\rangle \otimes|00\rangle+\left|\psi_{0 \epsilon}\right\rangle \otimes|0 \epsilon\rangle
$$

where the first factors are the position space amplitudes (or "channel amplitudes") corresponding to the indicates internal states of the two atoms. Thus the scattering problem we have to solve now involves solving the Schrdinger equation

$$
[E-H]\left|\Psi_{\vec{k}}\right\rangle=0
$$

with $H$ given by (3.4), the state $\left|\Psi_{\vec{k}}\right\rangle$ being of the form (3.5) and satisfying appropriate boundary conditions.

As a first step it is easy to rewrite this equation as a set of two coupled equations for the two channel amplitudes, by taking its scalar product successively with each of the two channel internal states. One obtains in this way

$$
\begin{aligned}
& {\left[E-\frac{p^{2}}{2 \mu_{R}}-V_{00}\right]\left|\psi_{00}\right\rangle=v_{c}\left|\psi_{0 \epsilon}\right\rangle} \\
& {\left[E-\epsilon-\frac{p^{2}}{2 \mu_{R}}-V_{0 \epsilon}\right]\left|\psi_{0 \epsilon}\right\rangle=v_{c}\left|\psi_{00}\right\rangle .}
\end{aligned}
$$

The channel potentials $V_{00}$ and $V_{0 \epsilon}$, and also the coupling potential $v_{c}$ are again assumed to vanish at large separations of the two atoms. Thus, if the total center of mass energy $E$ is less than $\epsilon$, the amplitude $\left|\psi_{0 \epsilon}\right\rangle$ will behave asymptotically as a decaying exponential, indicating that this channel is then a closed channel. Under these circumstances, the elastic scattering in the only open channel $|00\rangle$ can be described by a single equation of the form (3.1) with an appropriate effective potential $V$, which can now be derived from the coupled equations (3.6). The second equation can be formally solved for the closed channel amplitude giving

$$
\left|\psi_{0 \epsilon}\right\rangle=\frac{1}{E-\epsilon-\frac{p^{2}}{2 \mu_{R}}-V_{0 \epsilon}} v_{c}\left|\psi_{00}\right\rangle .
$$

Substituting this on the first equation yields

$$
\left[E-\frac{p^{2}}{2 \mu_{R}}-V_{00}-v_{c} \frac{1}{E-\epsilon-\frac{p^{2}}{2 \mu_{R}}-V_{0 \epsilon}} v_{c}\right]\left|\psi_{00}\right\rangle=0
$$

which identifies the appropriate effective potential for elastic scattering $V$ as the explicitly energy-dependent object

$$
V=V_{00}+v_{c} \frac{1}{E-\epsilon-\frac{p^{2}}{2 \mu_{R}}-V_{0 \epsilon}} v_{c}, \quad E<\epsilon .
$$

The inverse operator first introduced in eq. (3.7) can be expressed with the help of the eigenvectors of the closed channel hamiltonian $p^{2} / 2 \mu_{R}+V_{0 \epsilon}$. At negative channel energies one can have a discrete set of bound states $\left|\phi_{n}\right\rangle$ which vanish asymptotically and satisfy

$$
\begin{gathered}
{\left[\frac{p^{2}}{2 \mu_{R}}+V_{0 \epsilon}\right]\left|\phi_{n}\right\rangle=\eta_{n}\left|\phi_{n}\right\rangle,} \\
\eta_{n}<0, \quad\left\langle\phi_{n} \mid \phi_{n^{\prime}}\right\rangle=\delta_{n n^{\prime}}
\end{gathered}
$$

while at positive channel energies $\eta>0$ the spectrum is continuous and the eigenstates are scattering states $\left|\phi_{\eta}\right\rangle$ satisfying scattering boundary conditions and the equation

$$
\left[\frac{p^{2}}{2 \mu_{R}}+V_{0 \epsilon}\right]\left|\phi_{\eta}\right\rangle=\eta\left|\phi_{\eta}\right\rangle, \quad \eta=\frac{\hbar^{2} k^{2}}{2 \mu_{R}}>0 .
$$

The effective potential for elastic scattering can thus be written in terms of the closed channel eigenvectors as 


$$
V=V_{00}+\sum_{n} v_{c}\left|\phi_{n}\right\rangle \frac{1}{E-\epsilon-\eta_{n}}\left\langle\phi_{n}\left|v_{c}+\frac{1}{(2 \pi)^{3}} \int_{0}^{\infty} d^{3} k v_{c}\right| \phi_{\eta}\right\rangle \frac{1}{E-\epsilon-\eta}\left\langle\phi_{\eta}\right| v_{c}, \quad E<\epsilon .
$$

The denominator in the continuous spectrum part of this operator does not vanish as long as the $|0 \epsilon\rangle$ channel remains closed but, since $\eta_{n}<0$, the part associated with the discrete spectrum becomes singular at energies $E$ such that $E-\epsilon-\eta_{n}=0$, and is very strongly energy dependent in the vicinity of each one of these singularities.

\subsubsection{Single closed channel bound state and resonance scattering}

For the sake of simplicity, let us assume that there is just one bound state $\left|\phi_{0}\right\rangle$, with eigenvalue $-\epsilon<\eta_{0}<0$, so that a singularity in fact develops at $0<E=E_{0} \equiv \epsilon+\eta_{0}<\epsilon$, and hence in the energy interval corresponding to pure elastic scattering. How does the singularity affect the elastic scattering amplitude in its vicinity $E \sim E_{0}$ ?

In order to answer this question, note that the energy dependence coming from the contribution of the continuous spectrum is slow in comparison with that associated with the bound state singularity. Thus it is permissible to lump it together with the channel potential $V_{00}$ and define an effective "background" channel potential

$$
\tilde{V}_{00}=V_{00}+\frac{1}{(2 \pi)^{3}} \int_{0}^{\infty} d^{3} k v_{c}\left|\phi_{\eta}\right\rangle \frac{1}{E-\epsilon-\eta}\left\langle\phi_{\eta}\right| v_{c}
$$

which is only slowly energy dependent near $E=E_{0}$. The open channel amplitude then satisfies the equation

$$
\left[E-\frac{p^{2}}{2 \mu_{R}}-\tilde{V}_{00}\right]\left|\psi_{00 \vec{k}}\right\rangle=v_{c}\left|\phi_{0}\right\rangle \frac{1}{E-E_{0}}\left\langle\phi_{0}\left|v_{c}\right| \psi_{00 \vec{k}}\right\rangle
$$

where the asymptotic momentum $\vec{k}$ is related to $E$ as usual, i.e. $E=\hbar^{2} k^{2} / 2 \mu_{R}$. This equation has to be solved for $\left|\psi_{00 \vec{k}}\right\rangle$ with scattering asymptotic boundary conditions of the form (3.2).

This can be done in a variety of ways, of which we choose that which is possibly least dependent on invoking auxiliary results from scattering theory. The first step is to note that equation (3.8) is equivalent to the pair of coupled equations

$$
\begin{aligned}
& {\left[E-\frac{p^{2}}{2 \mu_{R}}-\tilde{V}_{00}\right]\left|\psi_{00 \vec{k}}\right\rangle=v_{c}\left|\phi_{0}\right\rangle \alpha} \\
& {\left[E-E_{0}\right] \alpha=\left\langle\phi_{0}\left|v_{c}\right| \psi_{00 \vec{k}}\right\rangle}
\end{aligned}
$$

since it can be recovered from them by eliminating the cnumber amplitude $\alpha$. The first of these equations appears as an inhomogeneous equation which can be solved by using the appropriate Green's function $G_{E}$ of the differential operator on the left hand side as

$$
\left|\psi_{00 \vec{k}}\right\rangle=\left|\chi_{00 \vec{k}}\right\rangle+G_{E} v_{c}\left|\phi_{0}\right\rangle \alpha
$$

where $\left|\chi_{00 \vec{k}}\right\rangle$ is a solution of the homogeneous form of the same equation. It describes elastic scattering by the background effective potential $\tilde{V}_{00}$ alone. Substitution into the second equation determines $\alpha$ as

$$
\alpha=\frac{\left\langle\phi_{0}\left|v_{c}\right| \chi_{00 \vec{k}}\right\rangle}{E-E_{0}-\left\langle\phi_{0}\left|v_{c} G_{E} v_{c}\right| \phi_{0}\right\rangle}
$$

leading therefore to the desired solution

$$
\left|\psi_{00 \vec{k}}\right\rangle=\left|\chi_{00 \vec{k}}\right\rangle+G_{E} v_{c}\left|\phi_{0}\right\rangle \frac{\left\langle\phi_{0}\left|v_{c}\right| \chi_{00 \vec{k}}\right\rangle}{E-E_{0}-\left\langle\phi_{0}\left|v_{c} G_{E} v_{c}\right| \phi_{0}\right\rangle} .
$$

The effect of the singularity at $E=E_{0}$ is entirely contained in the last term of this solution, which shows that the singularity itself is modified by the additional, also energy dependent term $\left\langle\phi_{0}\left|v_{c} G_{E} v_{c}\right| \phi_{0}\right\rangle$. In order to make the structure of this term more explicit, recall that the appropriate Green's function appearing there can be written as (cf. ref. [1], section 10.3)

$$
G_{E}=\int \frac{d^{3} k^{\prime}}{(2 \pi)^{3}}\left[\left|\chi_{00 \overrightarrow{k^{\prime}}}\right\rangle \frac{\mathcal{P}}{E-E^{\prime}}\left\langle\chi_{00 \overrightarrow{k^{\prime}}}|-i \pi| \chi_{00 \vec{k}}\right\rangle \delta\left(E-E^{\prime}\right)\left\langle\chi_{00 \vec{k}}\right|\right], \quad E^{\prime}=\frac{\hbar^{2} k^{2}}{2 \mu_{R}}
$$

where $\mathcal{P}$ denotes the principal value of the singular integral and the background scattering states have been assumed to be normalized as in eq. (3.2). This gives

$$
\left\langle\phi_{0}\left|v_{c} G_{E} v_{c}\right| \phi_{0}\right\rangle=\mathcal{P} \int \frac{d^{3} k^{\prime}}{(2 \pi)^{3}} \frac{\left|\left\langle\phi_{0}\left|v_{c}\right| \chi_{00 k^{\prime}}\right\rangle\right|^{2}}{E-E^{\prime}}-i \pi \int \frac{d^{3} k^{\prime}}{(2 \pi)^{3}}\left|\left\langle\phi_{0}\left|v_{c}\right| \chi_{00 \vec{k}}\right\rangle\right|^{2} \delta\left(E-E^{\prime}\right) \equiv \Delta_{0}-i \frac{\Gamma_{0}}{2} .
$$


Note that $\Delta_{0}$ and $\Gamma_{0}$ are real energy dependent quantities, their energy dependence coming from that of the background scattering states. We are thus led to

$$
\left|\psi_{00 \vec{k}}\right\rangle=\left|\chi_{00 \vec{k}}\right\rangle+G_{E} v_{c}\left|\phi_{0}\right\rangle \frac{\left\langle\phi_{0}\left|v_{c}\right| \chi_{00 \vec{k}}\right\rangle}{E-E_{0}-\Delta_{0}+i \frac{\Gamma_{0}}{2}}
$$

This solution shows that the effect of the singularity associated with the closed channel bound state appears as an additional component in the elastic scattering state which appears multiplied by a resonant complex factor. To the extent that the energy dependence of $\Delta_{0}$ and of $\Gamma_{0}$ can be ignored, the squared absolute value of this factor has a BreitWigner profile which peaks at the energy $E=E_{0}+\Delta_{0}$ and has a width $\Gamma_{0}$.

The various ingredients in the seemingly elaborate expression (3.10) can actually be interpreted in a rather transparent way. As already mentioned, the first term just represents the energy-smooth background elastic scattering. The second term carries the resonant contribution, which results from transitions mediated by the channel coupling interaction $v_{c}$ from the open channel to the of the bound state $\left.\phi_{0}\right\rangle$ in the closed channel. This is what the amplitude $\left\langle\phi_{0}\left|v_{c}\right| \chi_{00 \vec{k}}\right\rangle$ in the numerator of this term stands for. Once these transitions feed the bound state amplitude, it acts as a source for an additional amplitude in the open channel, this being given by the factor involving the background Green's function, $G_{E} v_{c}\left|\phi_{0}\right\rangle$. The energy dependent denominator accounts for the dynamics of the two-atom system while in transit through the closed channel or, in other words for the propagation of the system through the closed channel. It contains information about the energy of the bound state and takes into account the ever present possibility of leaking back to the open channel through the matrix element $\left\langle\phi_{0}\left|v_{c} G_{E} v_{c}\right| \phi_{0}\right\rangle$, which introduces the energy shift $\Delta_{0}$ and the width $\Gamma_{0}$. The latter also guarantees the enforcement of the time-energy uncertainty relation. In fact, the expression for the width can be written as

$$
\Gamma_{0}=2 \pi \int \frac{d^{3} k^{\prime}}{(2 \pi)^{3}}\left|\left\langle\phi_{0}\left|v_{c}\right| \chi_{00 \overrightarrow{k^{\prime}}}\right\rangle\right|^{2} \delta\left(E-E^{\prime}\right)
$$

so that $\Gamma_{0} / \hbar$ is just Fermi's Golden Rule expression for the transition rate out of the closed channel bound state to the open channel background continuum through the action of the channel coupling interaction $v_{c}$. Note however that this is here not merely a perturbative approximation, but an exact expression.

\subsubsection{Resonant phase shift in low energy scattering}

Our next task will be to extract from eq. (3.10) the effects of a very low energy resonance on the $s$-wave phase-shift and on the scattering amplitude. What is implied here by 'very low energy' is the effective suppression by their respective centrifugal barriers of all higher partial waves.

As in the case of eq. (3.3), the $s$-wave component of the background elastic scattering wavefunction appearing in the expression for the width (3.11) can be written as

$$
\left\langle\vec{r} \mid \chi_{00 \vec{k}}\right\rangle \equiv e^{i \tilde{\delta}_{0}(k)} \tilde{u}_{0 k}(r) \quad \stackrel{r \rightarrow \infty}{\longrightarrow} e^{i \tilde{\delta}_{0}} \frac{\sin \left(k r+\tilde{\delta}_{0}\right)}{k r}
$$

where $\tilde{\delta}_{0}$ is the background $s$-wave phase shift produced by the effective potential $\tilde{V}_{00}$ and the function $\tilde{u}_{0 k}(r)$ is independent of angular variables, regular at the origin and slowly varying with $k$. The matrix element which appears in the expression for the width is therefore independent of the angular components of $\overrightarrow{k^{\prime}}$. The energy delta function then allows one to perform the momentum integration explicitly with the result

$$
\Gamma_{0}=\frac{\mu_{R}}{\pi \hbar^{2}} k\left|\left\langle\phi_{0}\left|v_{c}\right| \tilde{u}_{0 k}\right\rangle\right|^{2} \equiv 2 \gamma k
$$

where a linear phase-space induced $k$-dependence has been made explicit and the newly defined "reduced width" parameter

$$
\gamma \equiv \frac{\mu_{R}}{2 \pi \hbar^{2}}\left|\left\langle\phi_{0}\left|v_{c}\right| \tilde{u}_{0 k}\right\rangle\right|^{2}
$$

is slowly varying with $k$. Note that this parameter has dimensions of energy times length.

We next extract an expression for the full $s$-wave phase shift $\delta_{0}$, including the resonant contribution, from the scattering state (3.10). To this effect we use an asymptotic expression for the $s$-wave Green's function $G_{E(l=0)}$ written in terms of the regular background scattering solution $\tilde{u}_{0 k}(r)$ which reads

$$
\left\langle r\left|G_{E(l=0)}\right| r^{\prime}\right\rangle \quad \stackrel{r \rightarrow \infty}{\longrightarrow} \quad-\frac{\mu_{R}}{2 \pi \hbar^{2}} \frac{e^{i k r}}{r} e^{i \tilde{\delta}_{0}} \tilde{u}_{0 k}\left(r^{\prime}\right)
$$

which gives, upon substitution in (3.10),

$$
\left\langle r \mid \psi_{00 k}\right\rangle \stackrel{r \rightarrow \infty}{\longrightarrow}-\frac{1}{2 i k r}\left[e^{-i k r}-e^{2 i \tilde{\delta}_{0}}\left(1-\frac{i \Gamma_{0}}{E-E_{0}-\Delta_{0}+i \frac{\Gamma_{0}}{2}}\right) e^{i k r}\right]
$$


from which we can read the $s$-wave $S$-matrix as

$$
e^{2 i \delta_{0}}=e^{2 i \tilde{\delta}_{0}}\left(\frac{E-E_{0}-\Delta_{0}-i \frac{\Gamma_{0}}{2}}{E-E_{0}-\Delta_{0}+i \frac{\Gamma_{0}}{2}}\right) \equiv e^{2 i \tilde{\delta}_{0}} e^{2 i \delta_{R}}
$$

where the resonant contribution $\delta_{R}$ to the phase shift is

$$
\tan \delta_{R}=-\frac{\Gamma_{0} / 2}{E-E_{0}-\Delta_{0}} .
$$

The characteristic behavior of the resonant phase shift for a narrow resonance, i.e., a resonance in which the energy dependence of $\Gamma_{0}$ and $\Delta_{0}$ can be ignored (recall that this en- ergy dependence comes from the "background" scattering involving the open channel alone) is shown in Fig. 3.1. The resonant phase $\delta_{R}$ grows most rapidly through $\delta_{R}=\pi / 2$ at the energy $E_{0}+\Delta_{0}$ for which the denominator vanishes. As the energy $E$ sweeps across the resonance, $\delta_{R}$ grows by $\sim \pi$, the change between $\pi / 4$ and $3 \pi / 4$ occurring in an energy interval of width $\Gamma_{0}$. Our concern here is however with cases in which the energy $E_{0}+\Delta_{0}$ is close enough to zero so that one can write the phase-shift in terms of the scattering length as $\tilde{\delta}_{0}+\delta_{R}=\delta_{0} \simeq-k a=-k\left(\tilde{a}+a_{R}\right)$, we obtain for the full scattering length, including the resonant contribution

$$
a=\tilde{a}+a_{R}=\tilde{a}+\lim _{k \rightarrow 0} \frac{1}{k} \tan ^{-1} \frac{\Gamma_{0} / 2}{E-E_{0}-\Delta_{0}}=\tilde{a}-\frac{\gamma}{E_{0}+\Delta_{0}} \equiv \tilde{a}-\frac{\gamma}{\varepsilon} .
$$

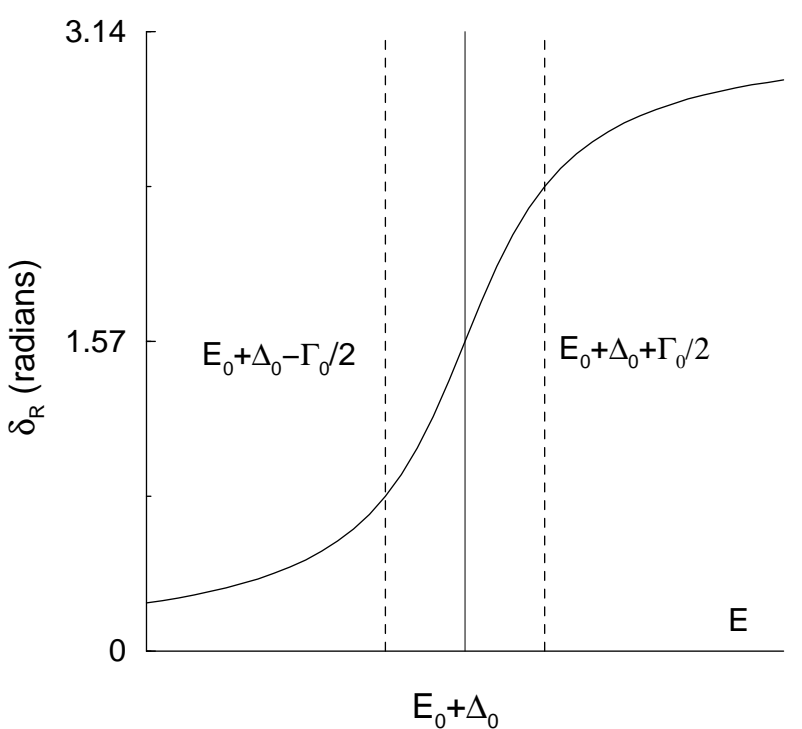

Figure 3.1. Resonant phase shift $\delta_{R}$ for a typical narrow resonance as a function of the energy $E$. The phase $\delta_{R}$ grows from $\pi / 4$ to $3 \pi / 4$ in an energy interval of width $\Gamma_{0}$.

The energy $\varepsilon \equiv E_{0}+\Delta_{0}$ marks the position of the resonance energy with respect to the threshold for elastic scattering and is usually referred to in this context as the "detuning" of the resonance.

This expression for the full scattering length shows how its "background" (off-resonance) value is affected by the resonant scattering. The parameters which determine the resonant modification of the scattering length are the reduced width $\gamma$ and the detuning $\varepsilon$. When the detuning is sufficiently small, the resonant modification may be dramatic not only for the magnitude of the scattering length, but also by changing its sign. Moreover, whenever the atomic states defining the open elastic scattering channel and the closed channel bound state $\left|\phi_{0}\right\rangle$ have different magnetic moments, in the sense that their energies vary differently under the influence of an external magnetic field of strength $B$, one can effectively change the detuning by changing $B$. Thus, if $\varepsilon_{0}$ is the detuning for $B=0$, including the linear Zeeman shifts, due to $B \neq 0$ one can write

$$
\varepsilon=\varepsilon_{0}+\Delta \mu B
$$

where $\Delta \mu$ is an effective, difference magnetic moment measuring how the detuning varies with $B$ as the elastic channel threshold and the closed channel bound states react differently to this field. In this case the full scattering length can be seen as a function of the magnetic field $B$

$$
a=\tilde{a}+\frac{\gamma / \Delta \mu}{B_{0}-B}=\tilde{a}\left(1+\frac{\Delta_{B}}{B_{0}-B}\right)
$$

where $B_{0}=-\varepsilon_{0} / \Delta \mu$ is the field strength at which the detuning vanishes, and $\Delta_{B} \equiv \gamma / \tilde{a} \Delta \mu$ characterizes the width of the resonance in terms of magnetic field strength.

Typical behaviors of the scattering length $a$ as a function of the external magnetic field $B$ in the neighborhood of the field value $B_{0}$ corresponding to zero detuning are shown in Fig. 3.2. The case represented on the left corresponds to a resonance in ${ }^{23} \mathrm{Na}$ for which $B_{0}=907 \mathrm{G}$ and $\Delta_{B}=1 \mathrm{G}$. This was the first Feshbach resonance to be observed through their effect on the properties of BoseEinstein condensates[3], which in particular confirmed a previously calculated value of the magnetic field width parameter $\Delta_{B}$. In the case represented on the right of the figure the off-resonance scattering length $\tilde{a}$ is negative, but becomes positive in a domain of values of $B>B_{0}$. This situation is in fact found in a resonance in ${ }^{85} \mathrm{Rb}$ for which $B_{0}=155 \mathrm{G}$ and $\Delta_{B}=11.5 \mathrm{G}$. This resonance has been studied by means of photoassociation spectroscopy[27] and then used in an experiment to control the strength of the effective atom-atom interaction in a cold sample of ${ }^{85} \mathrm{Rb}$ gas above the critical temperature for condensation[4]. By disturbing the system out of equilibrium and measuring the equilibration rate as a function of the magnetic field, it was 

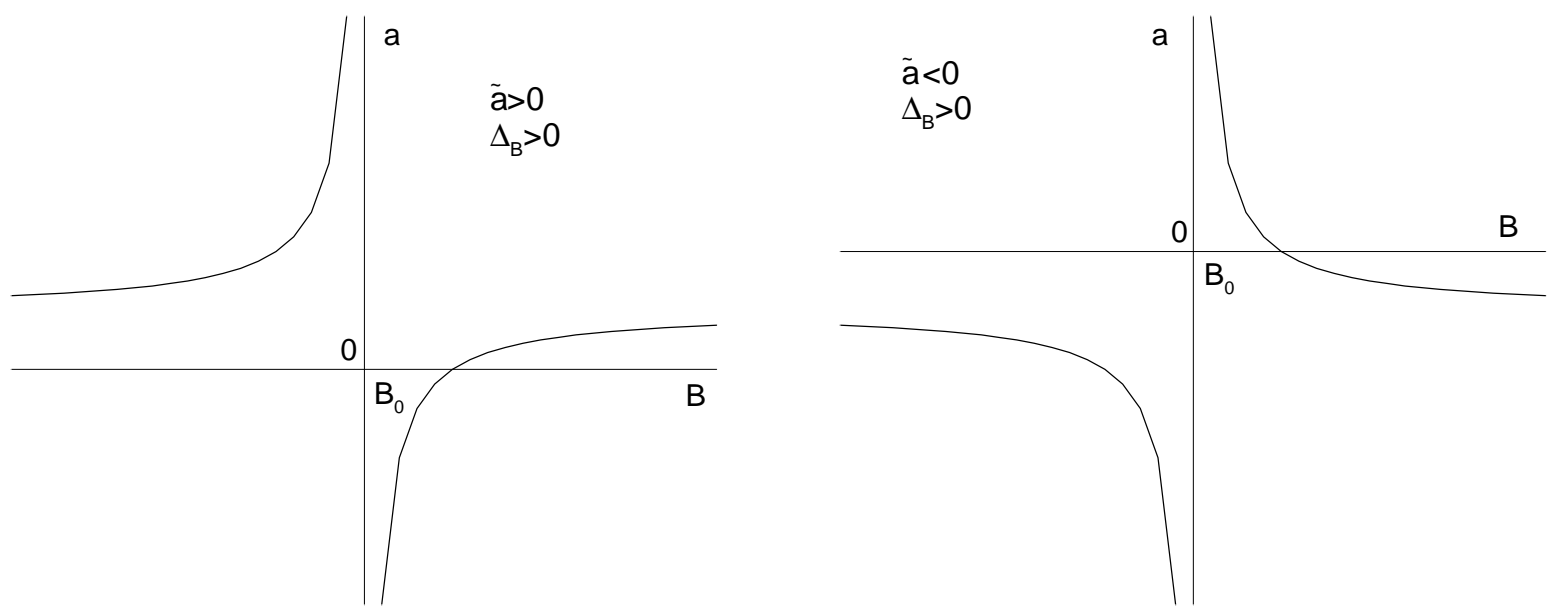

Figure 3.2. Left: typical behavior of the near resonance full scattering length when $\tilde{a}>0$ and $\Delta_{B}>0$, as for the resonance at $B_{0} \simeq 907$ $\mathrm{G}$ in ${ }^{23} \mathrm{Na}$. Right: same but for $\tilde{a}<0$, as for the resonance at $B_{0} \simeq 156 \mathrm{G}$ in ${ }^{85} \mathrm{Rb}$. Note changes of sign in both cases.

observed that the equilibration rate vanished at the field value for which $a=0$. This corresponds therefore to experimental realization of an ideal gas. This same resonance has been subsequently used to obtain stable condensates of ${ }^{85} \mathrm{Rb}$ atoms. A remarkable property of this resonance is that it has allowed for the formation of condensates with measured values of the scattering length in excess of as large as about 9000 Bohr radii[5].

Experimental and theoretical "Feshbach spectroscopy" studies have also been carried out for ${ }^{133} \mathrm{Cs}$, motivated both by attempts at using this type of atom for Bose-Einstein condensation and by the special role it plays as time and frequency standard[28, 29]. The scattering length for atoms in the lowest hyperfine state has the $B>B_{0}$ behavior shown on the left part of Fig. 3.2, with $B_{0}=-8 \mathrm{G}$. This negative value means that the resonant state does not cross the elastic scattering threshold as the field is increased from zero. The scattering length varies from $a \simeq-3000$ Bohr radii at zero magnetic field up to $a \simeq 1000$ Bohr radii at $B=55 \mathrm{G}$. It is observed to vanish for $B=17 \mathrm{G}[28,2]$. This is therefore a very broad resonance $\left(\Delta_{B} \simeq 25 \mathrm{G}\right)$ which allows for tuning the scattering length in a very broad range with small magnetic fields. This flexibility has in fact been crucial for achieving the Cs condensate, as it allowed to adjust the residual interaction in oder to optimize conditions for the various steps of the cooling process. Once the condensate was formed, adjustment of the external magnetic field to $17 \mathrm{G}$ led to an experimental "ideal" condensate.

Finally, it should be noted explicitly that the property $a=0$ results in fact from a cancellation between two contributions to the scattering amplitude which are of a different nature, namely the effective potential scattering in the elastic channel and the resonant contribution involving virtual transfers to the closed inelastic channel and back. thus, even though the wavefunction of a scattering pair of atoms is asymptotically indistinguishable from the wavefunction of a free pair, it is entirely different from the latter within the interaction region. The "ideal gas" behavior results therefore from insensitivity of the relevant properties of dilute systems to the outwardly healed interaction wounds.

\subsection{Feshbach resonances and molecular con- densates}

The mechanism underlying Feshbach resonant effects in elastic atom-atom scattering involves the formation of twoatom states of molecular type, which are not stationary states on account of their coupling to the open elastic scattering channel. The most naive form of bringing this process to bear in the context of the many-body problem is to use the resonance modified scattering length to write the two-body effective interaction between atoms. This is in fact what is implicitly or even explicitly assumed in many cases, without meeting any gross inconsistencies on the experimental side.

However, it was soon realized that one should be dealing in such cases with richer many-body dynamics, in which in the neighborhood of a Feshbach resonance a dynamic equilibrium situation may be reached in the many-body system involving dimer formation and decay back to the elastic channel, leading do condensates hybridized by the presence of a molecular, or dimer phase. This suggestion has in fact been made and elaborated in terms of a definite theoretical model by Timmermans et al. in ref. [26]. As will be discussed shortly, this model possibly leads to results which may not fully agree with the naive point of view, signaling that at least a more detailed understanding of the atom-atom effective interaction in the presence of resonant effects is needed[30].

The model adopted by Timmermans et al. basically implements coupled equations analogous to (3.9) in a manybody context, allowing for the conventional treatment of the effective two-body interaction. This is achieved by introducing into the second quantized hamiltonian a new field operator $\psi_{b}(\vec{r})$ associated with the dimer state $\left|\phi_{0}\right\rangle$ and commuting with the field operator associated with the single atoms, written as $\psi_{a}(\vec{r})$. The coupling of a pair of atoms to the dimmer state, described by the coupling potential $v_{c}$ in eqs. (3.9), is represented in the hamiltonian density by an interaction term of the form 


$$
\mathcal{H}_{\text {int }}(\vec{r})=\alpha\left(\psi_{b}^{\dagger}(\vec{r}) \psi_{a}(\vec{r}) \psi_{a}(\vec{r})+\psi_{a}^{\dagger}(\vec{r}) \psi_{a}^{\dagger}(\vec{r}) \psi_{b}(\vec{r})\right) .
$$

This term represents an effective contact interaction of strength $\alpha$ which either converts a pair of atoms into a dimer or, conversely, transforms a dimer back into a pair of atoms.
The constant $\alpha$ is related to the reduced width $\gamma$ of the involved Feshbach resonance as $\alpha^{2}=2 \pi \hbar^{2} \gamma / M$.

Additional terms of the effective hamiltonian density are analogous to eq. (2.5) for each of the two fields, including the detuning parameter $\varepsilon$ and an additional effective twobody interaction term involving different fields. They read

$$
\begin{aligned}
\mathcal{H}_{a}(\vec{r})+\mathcal{H}_{b}(\vec{r})+\mathcal{H}_{a b}(\vec{r}) & =\psi_{a}^{\dagger}(\vec{r})\left(-\frac{\hbar^{2} \nabla^{2}}{2 M}+V_{a}(\vec{r})\right) \psi_{a}(\vec{r})+\frac{\lambda_{a}}{2} \psi_{a}^{\dagger}(\vec{r}) \psi_{a}^{\dagger}(\vec{r}) \psi_{a}(\vec{r}) \psi_{a}(\vec{r})+ \\
& +\psi_{b}^{\dagger}(\vec{r})\left(-\frac{\hbar^{2} \nabla^{2}}{4 M}+\varepsilon+V_{b}(\vec{r})\right) \psi_{b}(\vec{r})+\frac{\lambda_{b}}{2} \psi_{b}^{\dagger}(\vec{r}) \psi_{b}^{\dagger}(\vec{r}) \psi_{b}(\vec{r}) \psi_{b}(\vec{r})+ \\
& +\lambda_{a b} \psi_{b}^{\dagger}(\vec{r}) \psi_{a}^{\dagger}(\vec{r}) \psi_{a}(\vec{r}) \psi_{b}(\vec{r})
\end{aligned}
$$

the effective hamiltonian being therefore

$$
H_{\mathrm{eff}}=\int d^{3} r\left[\mathcal{H}_{a}(\vec{r})+\mathcal{H}_{b}(\vec{r})+\mathcal{H}_{a b}(\vec{r})+\mathcal{H}_{\text {int }}(\vec{r})\right]
$$

Note that the mass of the dimers has been written as being twice the mass of the atoms, and different trap potentials have been allowed for each of these two species. It can be also easily verified that the operator

$$
\hat{N}=\hat{N}_{a}+2 \hat{N}_{b}=\int d^{3} r \psi_{a}^{\dagger}(\vec{r}) \psi_{a}(\vec{r})+2 \int d^{3} r \psi_{b}^{\dagger}(\vec{r}) \psi_{b}(\vec{r})
$$

is a constant of motion of $H_{\text {eff. }}$. Since each dimer accounts for a pair of atoms, this operator represents the total number of atoms present in the system in either form. Since $\hat{N}_{a}$ and $\hat{N}_{b}$ by themselves are not constants of motion, we may conclude that stationary states of the hybrid system will in general involve correlated quantum fluctuations of the two species which however become negligible in the case of a uniform system (i.e. $V_{a}=V_{b}=0$ ) when one takes the thermodynamic limit[31]. In order to obtain a simple mean-field approximation of the Gross-Pitaevski type one must therefore discard also correlations between fluctuating numbers of atoms and dimers. This is usually done by means of a coherent ansatz[26, 32]

$$
\left|u_{a} u_{b}\right\rangle=e^{\int d^{3} r\left(u_{a}(\vec{r}) \psi_{a}^{\dagger}(\vec{r})-u_{a}^{*}(\vec{r}) \psi_{a}(\vec{r})\right)} e^{\int d^{3} r\left(u_{b}(\vec{r}) \psi_{b}^{\dagger}(\vec{r})-u_{b}^{*}(\vec{r}) \psi_{b}(\vec{r})\right)}|0\rangle
$$

which leads to an energy functional of the form

$$
\begin{aligned}
\left\langle u_{a} u_{b}\left|H_{\mathrm{eff}}\right| u_{a} u_{b}\right\rangle= & \int d^{3} r u_{a}^{*}(\vec{r})\left(-\frac{\hbar^{2} \nabla^{2}}{2 M}+V_{a}\right) u_{a}(\vec{r})+\int d^{3} r u_{b}^{*}(\vec{r})\left(-\frac{\hbar^{2} \nabla^{2}}{4 M}+\varepsilon+V_{b}\right) u_{b}(\vec{r})+ \\
& +\frac{\lambda_{a}}{2} \int d^{3} r\left|u_{a}(\vec{r})\right|^{4}+\frac{\lambda_{b}}{2} \int d^{3} r\left|u_{b}(\vec{r})\right|^{4}+\lambda_{a b} \int d^{3} r\left|u_{a}(\vec{r})\right|^{2}\left|u_{b}(\vec{r})\right|^{2}+ \\
& +\alpha \int d^{3} r\left(u_{b}^{*}(\vec{r}) u_{a}(\vec{r})^{2}+u_{b}(\vec{r}) u_{a}^{*}(\vec{r})^{2}\right) .
\end{aligned}
$$

Variation with respect to the amplitudes $u_{a}(\vec{r})$ and $u_{b}(\vec{r})$ with the constraint on the total number taken care of by means of a Lagrange multiplier $\mu$ now yields Gross-Pitaevski coupled equations

$$
\begin{aligned}
& \left(-\frac{\hbar^{2} \nabla^{2}}{2 M}+V_{a}+\lambda_{a}\left|u_{a}(\vec{r})\right|^{2}+\lambda_{a b}\left|u_{b}(\vec{r})\right|^{2}\right) u_{a}(\vec{r})+2 \alpha u_{b}(\vec{r}) u_{a}^{*}(\vec{r})=\mu u_{a}(\vec{r}) \\
& \left(-\frac{\hbar^{2} \nabla^{2}}{4 M}+\varepsilon+V_{b}+\lambda_{b}\left|u_{b}(\vec{r})\right|^{2}+\lambda_{a b}\left|u_{a}(\vec{r})\right|^{2}\right) u_{b}(\vec{r})+\alpha u_{a}^{2}(\vec{r})=2 \mu u_{b}(\vec{r})
\end{aligned}
$$

with $\mu$ determined by the subsidiary condition on the total mean number of atoms

$$
\langle\hat{N}\rangle \equiv\left\langle u_{a} u_{b}|\hat{N}| u_{a} u_{b}\right\rangle=\left\langle\hat{N}_{a}\right\rangle+2\left\langle\hat{N}_{b}\right\rangle=\int d^{3} r\left(\left|u_{a}(\vec{r})\right|^{2}+2\left|u_{b}(\vec{r})\right|^{2}\right)=N .
$$

The mean squared dispersion in the total mean number of atoms introduced through the coherent ansatz can also be easily evaluated. It is different from zero, and results from the combined uncorrelated fluctuations of $\hat{N}_{a}$ and $\hat{N}_{b}$ : 


$$
\sigma_{N}^{2}=\left\langle\hat{N}^{2}\right\rangle-\langle\hat{N}\rangle^{2}=\left\langle\hat{N}_{a}^{2}\right\rangle+4\left\langle\hat{N}_{b}^{2}\right\rangle-\left\langle\hat{N}_{a}\right\rangle^{2}-4\left\langle\hat{N}_{b}\right\rangle^{2}=\sigma_{N_{a}}^{2}+4 \sigma_{N_{b}}^{2} .
$$

Alternatively, one may allow the displacement functions $u_{a}$ and $u_{b}$ of the coherent ansatz (3.14) to be time dependent and obtain the time dependent generalization of the coupled Gross-Pitaevski equations (3.15) by using the time-dependent variational principle

$$
\delta \int d t\left(i \hbar\left\langle u_{a} u_{b}\left|\frac{\partial}{\partial t}\right| u_{a} u_{b}\right\rangle-\left\langle u_{a} u_{b}\left|H_{\mathrm{eff}}\right| u_{a} u_{b}\right\rangle\right)=0,
$$

where variations are to be taken with respect to $u_{a}(\vec{r}, t)$ and $u_{a}(\vec{r}, t)$. One obtains in this way

$$
\begin{aligned}
i \hbar \frac{\partial u_{a}(\vec{r}, t)}{\partial t} & =\left(-\frac{\hbar^{2} \nabla^{2}}{2 M}+V_{a}+\lambda_{a}\left|u_{a}(\vec{r})\right|^{2}+\lambda_{a b}\left|u_{b}(\vec{r})\right|^{2}\right) u_{a}(\vec{r})+2 \alpha u_{b}(\vec{r}) u_{a}^{*}(\vec{r}) \\
i \hbar \frac{\partial u_{b}(\vec{r}, t)}{\partial t} & =\left(-\frac{\hbar^{2} \nabla^{2}}{4 M}+\varepsilon+V_{b}+\lambda_{b}\left|u_{b}(\vec{r})\right|^{2}+\lambda_{a b}\left|u_{a}(\vec{r})\right|^{2}\right) u_{b}(\vec{r})+\alpha u_{a}^{2}(\vec{r})
\end{aligned}
$$

This time dependent mean field approximation conserves the total mean number of atoms, since equations (3.16) imply

$$
\frac{\partial}{\partial t} \int d^{3} r\left(\left|u_{a}(\vec{r}, t)\right|^{2}+2\left|u_{b}(\vec{r}, t)\right|^{2}\right)=0 .
$$

Thus the mean number prescribed by an initial condition will be preserved at all subsequent times.

The stationary equations (3.15) may be easily solved (particularly in the Thomas-Fermi approximation) for a variety of parameter values. Independently of this, however, their form alone reveals that, although a pure dimer stationary solution is possible $\left(u_{a} \equiv 0, u_{b} \neq 0\right)$, there can be no stationary solution in which $u_{b} \equiv 0$, i.e., a dimer component must always be present. This follows from the fact that in this case the second equation requires that one also must have $u_{a} \equiv 0$. The dimer fraction in the ground state depends on the detuning, and therefore can be varied by changing the value of an external magnetic field. If the detuning is large and negative, eqs. (3.15) reasonably predict large dimer fraction, in possible conflict with the naive point of view, according to which the scattering amplitude should simply approach its background value.

A particular case of the time-dependent equations (3.16) that can be solved in a relatively simple way is that of a spatially uniform system (so that one must have $V_{a}=V_{b}=0$ ) which remains uniform at all times, so that the functions $u_{a}$ and $u_{b}$ are time-dependent complex constants. The dynamical equations they satisfy are then

$$
\begin{aligned}
i \hbar \frac{d u_{a}}{d t} & =\left(\lambda_{a}\left|u_{a}(t)\right|^{2}+\lambda_{a b}\left|u_{b}(t)\right|^{2}\right) u_{a}(y)+2 \alpha u_{b}(t) u_{a}^{*}(t) \\
i \hbar \frac{d u_{b}}{d t} & =\left(\varepsilon+\lambda_{b}\left|u_{b}(t)\right|^{2}+\lambda_{a b}\left|u_{a}(t)\right|^{2}\right) u_{b}(t)+\alpha u_{a}^{2}(t) .
\end{aligned}
$$

The complex character of the amplitudes is a direct consequence of the coherent ansatz (3.14), which allows for fixing the phase of each of the two condensates. Writing $u_{a, b}=\left|u_{a, b}\right| e^{i \varphi_{a, b}}$, inspection of the equations of motion shows that only the relative phase $\varphi \equiv 2 \varphi_{a}-\varphi_{n}$ is dynamically relevant. Moreover, since the total atom number density $n \equiv\left|u_{a}\right|^{2}+2\left|u_{b}\right|^{2} \mid$ is a constant of motion, one express $u_{a} \mid$ and $\left|u_{b}\right|$ in terms of $n$ and a single additional variable $f$, which can be conveniently defined as

$$
f \equiv \frac{2\left|u_{b}\right|^{2}-\left|u_{a}\right|^{2}}{n}, \quad-1 \leq f \leq 1 .
$$

The variable $f$ describes the splitting of the total atom number density into atomic and molecular densities, the limiting values corresponding respectively to all-atoms and to allmolecules situations. It turns out to be an action variable canonically conjugated to the angle variable corresponding to the relevant relative phase $\varphi$. In fact, the equations of motion for the uniform, hybrid system can be re-expressed in terms of $f$ and $\varphi$ as[31]

$$
\begin{aligned}
\frac{d f}{d t} & =\frac{2 \alpha \sqrt{n}}{\hbar}(1-f) \sqrt{(1+f)} \sin \varphi \\
\frac{d \varphi}{d t} & =\frac{\varepsilon}{\hbar}-\frac{n \lambda_{a}}{\hbar}(1-f)+\frac{n \lambda_{b}}{4 \hbar}(1+f)-\frac{n \lambda_{a b}}{\hbar} f-\frac{\alpha \sqrt{n}}{\hbar} \frac{1+3 f}{\sqrt{1+f}} \cos \varphi
\end{aligned}
$$

It is easy to check that these equations are in fact classical hamiltonian equations corresponding to the hamiltonian function

$$
h \equiv \frac{\varepsilon}{\hbar} f+\frac{n \lambda_{a}}{2 \hbar}(1-f)^{2}+\frac{n \lambda_{b}}{8 \hbar}(1+f)^{2}+\frac{n \lambda_{a b}}{2 \hbar}\left(1-f^{2}\right)+\frac{2 \alpha \sqrt{n}}{\hbar}(1-f) \sqrt{1+f} \cos \varphi .
$$


The curves $h=$ constant in the $f \times \varphi$ plane are the phasespace trajectories of the solutions of eqs. (3.17).

On the experimental side, perhaps the most conspicuous signal of the nearby presence of a Feshbach resonance in the pioneering experiment of Inouye et al.[3] was an enhanced loss of atoms, leading to the complete loss of the system. The mechanisms taken to be responsible for such loss actually involve internal atomic degrees of freedom which are not considered explicitly in the simple two-channel model. Under static external field conditions, the dimer component relates in fact to a highly excited vibrational molecular state in the closed channel, which can be easily de-excited in a collision with another atom[33]. This is in fact an enhanced three-body recombination process due to the formation of the resonant, longer lived dimer. The kinetic energy released in this process leads to the loss of the atoms involved. In the case of the ${ }^{85} \mathrm{Rb}$ Feshbach resonance the role of this loss mechanism was much less important, possibly due to the low density of the condensate in this case. In fact, a recent experimental success relating to this system now strengthens the dimer hybridization picture. As already observed in ref. [26], a sudden change of this field should cause the two coherent phases to be out of equilibrium, leading to subsequent oscillations of the dimmer (and of the atom) fraction in time, as found from the time-dependent generalization (3.16) of the Gross-Pitaevski coupled equations and also seen in the particular case of eqs. (3.17). This type of oscillation with a frequency which depends on the detuning $\varepsilon$. and hence on the applied magnetic field $B$, has in fact been observed near the $B_{0}=156 \mathrm{G}$ resonance in ${ }^{85} \mathrm{Rb}$ [34]. The oscillations were however observed in the atomic component, the molecular component being experimentally very elusive.

Just recently, a different line of experiments succeeded in directly observing the formation of molecules[35] and also of molecular condensates mediated by a Feshbach resonances in systems of cold fermionic atoms[36, 37]. Due to the requirements of Fermi statistics, the cooling of fermionic atoms requires systems containing a mixture of two components (which may be e.g. two different spin states) in order to allow for the $s$-wave interactions. The experiments in refs. [35, 36] used ${ }^{40} \mathrm{~K}$ atoms, and that in ref. [37] use ${ }^{6} \mathrm{Li}$ atoms. Bose-Einstein condensation of a molecular phase in this case gives rise to a very peculiar system involving the equilibrium of this condensate with a two-component Fermi gas. Some theory of such systems is today in the early stages of development[38].

\subsection{Molecules in atomic condensates and hy- brid atomic condensates by stimulated transitions}

Stimulated electromagnetic transitions (Raman or otherwise contrived) provide a versatile tool to manipulate internal atomic degrees of freedom in atomic condensates, besides being many times used also in their production process. In this final section we describe briefly a process of coherent stimulated recombination of atoms which produces specific, "cold" molecular states[39] and discuss the coupled dynamics of a two-species atomic condensate in the presence of stimulated inter-species transitions[40, 41].

\subsection{Molecule formation}

Bound molecular states such as $|m\rangle$ (see Fig. 3.3) in the elastic channel which is open for the condensate atoms (labeled as " $a+a$ " in the figure) are not significantly populated by spontaneous electromagnetic transitions. They will however have important dipole coupling to bound states in closed and rather high-lying channels involving an appropriate electronic excitation in one of the atoms (such as the channel labeled " $a+a^{*}$ " in the same figure), and these will in turn dipole-couple down to bound molecular states $|m\rangle$ in the elastic channel. This second-order coupling can be stimulated by properly tuned external radiation sources, leading effectively to the decay of the zero relative energy continuum state to the bound molecular state.

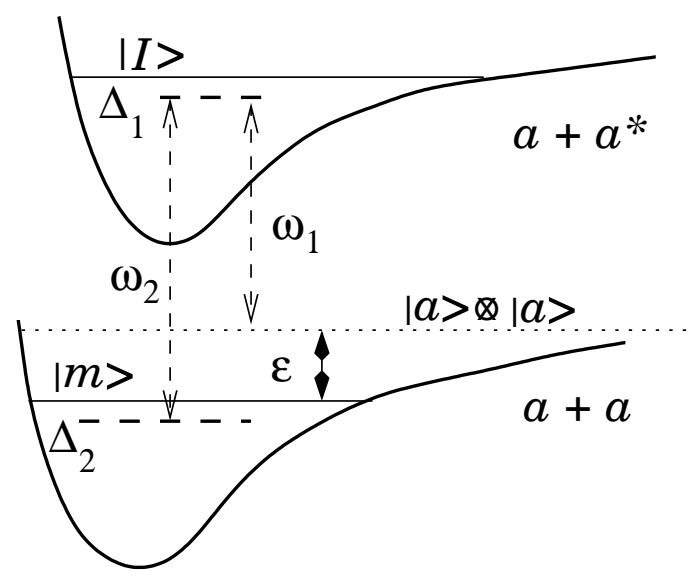

Figure 3.3. Stimulated second-order process leading from a state containing a pair of atoms $|a\rangle \otimes|a\rangle$ to a molecular state $|m\rangle$ in the " $a+a$ " channel through the intermediate state $|I\rangle$ in the dipolecoupled " $a+a^{*}$ " channel. The molecular state has binding energy $\varepsilon, \omega_{1}$ and $\omega_{2}$ are the frequencies of the external sources, which imply detunings $\Delta_{1}$ and $\Delta_{2}$.

The experiment described in ref. [39] uses thus procedure in a condensate of magnetically trapped ${ }^{87} \mathrm{Rb}$ atoms in the $f=1, m_{f}=-1$ hyperfine state, the intermediate state involving an electronic excitation of one of the atoms from $S$ to the $P$ level. Molecule formation was detected as an increase in the loss of atoms after exposure to the stimulating fields when $\omega_{2}-\omega_{1}$ was close to the value of the binding energy $\varepsilon$. The loss has been understood as due to molecule formation and subsequent escape from the trap. The molecules in this experiment are produced essentially "at rest", allowing for unprecedented accuracy in measuring the molecular binding energy. Interesting side many-body effects are an observed density dependence of the value of $\omega_{2}-\omega_{1}$ for maximum loss $(630.020 \mathrm{MHz}$ at a peak density of $0.77 \times 10^{14} \mathrm{~cm}^{-3}$ and $630.023 \mathrm{MHz}$ at $2.6 \times 10^{14} \mathrm{~cm}^{-3}$ ) and of the line shape, which becomes broader at larger values of the condensate density.

The system of atoms and molecules coupled by the external driving fields can be described by equations of motion of the form used in connection with the Feshbach resonances, eqs. 3.16, with an appropriate reinterpretation of 
parameters. The density of molecules $|m\rangle$ is described in terms of the amplitude $u_{b}(\vec{r})$. The constant $\alpha$ in the term that couples atoms and molecules and the detuning $\varepsilon$ (not to be confused here with the molecular binding energy!) are now set by the intensities and frequencies of the external driving fields, and therefore in principle completely "tunable". To bring the model closer to real experimental situations loss mechanisms can be introduced phenomenologically into the coupled equations[26, 31].

\subsubsection{Hybrid atomic condensates}

Second order stimulated processes can also couple two different hyperfine states of the condensate atoms, as illustrated in Fig. 3.4 for the case studied in refs. [40]. When a driving pulse is applied one may end up with a system involving two distinguishable types of atom which may be described by a hamiltonian density of the form

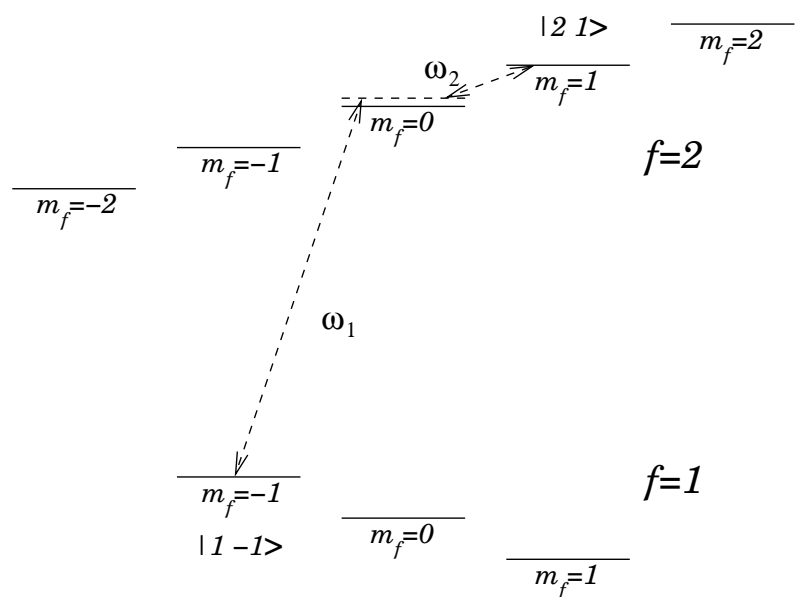

Figure 3.4. Stimulated second-order process coupling the hyperfine state $\left|f=1, m_{f}=-1\right\rangle$ to $\left|f=2, m_{f}=1\right\rangle$ in ${ }^{87} \mathrm{Rb}$. The frequencies are $\omega_{1} \simeq 6.8 \mathrm{GHz}$ and $\omega_{2} \simeq 2 \mathrm{MHz}$, in the microwave and radiofrequency ranges respectively. The state $\left|f=2, m_{f}-0\right\rangle$ acts as an intermediate state in the second order stimulated process.

$$
\mathcal{H}_{0}=\sum_{i=1}^{2} \psi_{i}^{\dagger}(\vec{r})\left[-\frac{\hbar^{2} \nabla^{2}}{2 M}+\epsilon_{i}+V_{i}(\vec{r})+\psi_{i}^{\dagger}(\vec{r}) \frac{4 \pi \hbar^{2} a_{i}}{2 M} \psi_{i}(\vec{r})\right] \psi_{i}(\vec{r})+\psi_{1}^{\dagger}(\vec{r}) \psi_{2}^{\dagger}(\vec{r}) \frac{4 \pi \hbar^{2} a_{12}}{M} \psi_{2}(\vec{r}) \psi_{1}(\vec{r})
$$

where $\psi_{1}(\vec{r})$ and $\psi_{2}(\vec{r})$ are field operators corresponding to the two different atomic species, the $a_{i}$ are their associated scattering lengths and $a_{12}$ is the scattering length describing the scattering of different species. Note that the two species may interact differently with the trapping arrangement, leading possibly to species-dependent trap potentials. Since the number of atoms of each species is in this case a constant of motion, the presence of the internal energy parameters $\epsilon_{i}$ is not dynamically relevant.

If, on the other hand, the external driving fields are kept on continuously, one needs also an interaction hamiltonian describing the coupling of the two species, which can be written as

$$
\mathcal{H}_{\text {int }}=\alpha\left(\psi_{1}^{\dagger}(\vec{r}) \psi_{2}(\vec{r})+\psi_{2}^{\dagger}(\vec{r}) \psi_{1}(\vec{r})\right), \quad \mathcal{H}=\mathcal{H}_{0}+\mathcal{H}_{\text {int }}
$$

With this extra term in the hamiltonian the number of atoms of each of the two species is no longer a constant of motion. Since the total number of atoms is conserved, one will in general have correlated quantum fluctuations of the two constituent populations, similarly to what has been discussed in connection with the coexistence of atoms and molecules in section 3.2. In this case the internal energy parameters $\epsilon_{i}$ acquire dynamical relevance as they affect the equilibrium mean values of the population fractions. Together with the coupling strength $\alpha$, they are determined by the driving field frequencies and intensities.

A possible mean field approximation of the GrossPitaevski type for this system can be obtained by using a coherent ansatz similar to eq. (3.14), i.e.

$$
\left|u_{1} u_{2}\right\rangle=e^{\int d^{3} r\left(u_{1}(\vec{r}) \psi_{1}^{\dagger}(\vec{r})-u_{1}^{*}(\vec{r}) \psi_{1}(\vec{r})\right)} e^{\int d^{3} r\left(u_{2}(\vec{r}) \psi_{2}^{\dagger}(\vec{r})-u_{2}^{*}(\vec{r}) \psi_{2}(\vec{r})\right)}|0\rangle .
$$

Using this ansatz the energy functional has the same form as the hamiltonian $H=\int d^{3} r \mathcal{H}(\vec{r})$ with the field operators (and their adjoints) replaced by the c-number functions $u_{i}(\vec{r})$ (and their complex-conjugates). The average total number of atoms is, on the other hand, 


$$
\left\langle u_{1} u_{2}\left|\int d^{3} r \sum_{i=1}^{2} \psi_{i}^{\dagger}(\vec{r}) \psi_{i}(\vec{r})\right| u_{1} u_{2}\right\rangle=\int d^{3} r \sum_{i=1}^{2}\left|u_{i}(\vec{r})\right|^{2}
$$

Fluctuations in the total number appear as a result of ignoring the correlation in the number fluctuations of the two species. Variation of the energy functional using a Lagrange multiplier $\mu$ to take the subsidiary condition regarding the average total number of atoms into account gives the now very symmetric extremum coupled equations

$$
\begin{aligned}
& {\left[-\frac{\hbar^{2} \nabla^{2}}{2 M}+\epsilon_{1}+V_{1}(\vec{r})+\frac{4 \pi \hbar^{2} a_{1}}{M}\left|u_{1}(\vec{r})\right|^{2}+\frac{4 \pi \hbar^{2} a_{12}}{M}\left|u_{2}(\vec{r})\right|^{2}\right] u_{1}(\vec{r})+\alpha u_{2}(\vec{r})=\mu u_{1}(\vec{r})} \\
& {\left[-\frac{\hbar^{2} \nabla^{2}}{2 M}+\epsilon_{2}+V_{2}(\vec{r})+\frac{4 \pi \hbar^{2} a_{2}}{M}\left|u_{2}(\vec{r})\right|^{2}+\frac{4 \pi \hbar^{2} a_{12}}{M}\left|u_{1}(\vec{r})\right|^{2}\right] u_{2}(\vec{r})+\alpha u_{1}(\vec{r})=\mu u_{2}(\vec{r})}
\end{aligned}
$$

A simple alternate ansatz which explicitly preserves the conservation of the total number of atoms exists for condensates involving two atomic species, however. It is written in terms of the hybrid creation operator

$$
a^{\dagger} \equiv \frac{1}{\sqrt{N}} \int d^{3} r\left(u_{1}(\vec{r}) \psi_{1}^{\dagger}(\vec{r})+u_{2}(\vec{r}) \psi_{2}^{\dagger}(\vec{r})\right)
$$

in which the factor $N^{-1 / 2}$ has been introduced for later convenience. Note that, with this definition, the condition

$$
\int d^{3} r\left(\left|u_{1}(\vec{r})\right|^{2}+\left|u_{2}(\vec{r})\right|^{2}\right)=N
$$

gives for the annihilation operator $a$ and its adjoint the standard boson commutation relation $\left[a, a^{\dagger}\right]=1$. This leads then to the $N$-atom ansatz

$$
\left|N ; u_{1} u_{2}\right\rangle \equiv \frac{1}{\sqrt{N !}}\left(a^{\dagger}\right)^{N}|0\rangle .
$$

Evaluation of the energy functional with this ansatz is straightforward using the commutation relation $\left[\psi_{i}(\vec{r}), a^{\dagger}\right]=u_{i}(\vec{r}) / \sqrt{N}$. The result is essentially the same as that obtained in the case of the coherent ansatz, the difference being just additional factors $N(N-1) / N^{2}$ in the two-body terms involving $a_{1}$ and $a_{2}$. Variation of the functional with what is now the normalization condition (3.20) accounted for in terms of a Lagrange multiplier $\mu$ leads therefore again to the equations (3.19), albeit with the third terms on the left hand side multiplied by $(N-1) / N$.

Thus when $N$ is large the functions $u_{i}(\vec{r})$ are determined from the same set of equations. In the case of the sharp number ansatz (3.21), however, one can evaluate a possibly meaningful fluctuation in the mean number of one of the two species. To this effect use

$$
\left\langle N ; u_{1} u_{2}\left|\hat{N}_{i}\right| N ; u_{1} u_{2}\right\rangle=\int d^{3} r\left|u_{i}(\vec{r})\right|^{2} \equiv\left\langle N_{i}\right\rangle
$$

and

$$
\left\langle N ; u_{1} u_{2}\left|\hat{N}_{i}^{2}\right| N ; u_{1} u_{2}\right\rangle=\frac{N-1}{N}\left\langle N_{i}\right\rangle^{2}+\left\langle N_{i}\right\rangle
$$

to obtain

$$
\sigma_{N_{i}}^{2}=\left\langle N_{i}\right\rangle\left(1-\frac{\left\langle N_{i}\right\rangle}{N}\right)
$$

which shows that the number fluctuation is in fact reduced with respect to the uncorrelated poissonian fluctuations associated with the coherent ansatz. The reduction factor depends on the fraction of the atoms which on the average are in each of the two coupled internal states, and therefore does not go away as $N$ becomes very large.

When the ansatz (3.21) is used, the functions $u_{i}(\vec{r})$ describe the spatial distribution of the internal hybridization of each and all of the $N$ atoms, which as a result of the ("Brueckner") mean-field approximation occupy the same single-particle state. This coherent internal superposition takes the place of the coherence of "two condensates" which one is led to consider when using the coherent ansatz.

The solution of equations (3.19) is very simple in the case of a uniform system, but even so the results are not entirely trivial. For definiteness we adopt the point of view of the sharp number ansatz (3.21). The functions $u_{i}(\vec{r})$ are now complex constants which it is convenient to write in polar form as

$$
u_{i}(\vec{r}) \rightarrow \sqrt{\frac{N}{\mathcal{V}}} x_{i} e^{i \varphi_{i}}
$$

where $\mathcal{V}$ is a quantization volume. Furthermore, the normalization condition (3.20) can be taken care of explicitly by parameterizing the $x_{i}$ in terms of a mixing angle $\theta$ as

$$
x_{1}=\cos \theta, \quad x_{2}=\sin \theta .
$$

With these simplifications it is preferable to work directly with the energy functional rather than with the coupled equations. It becomes 

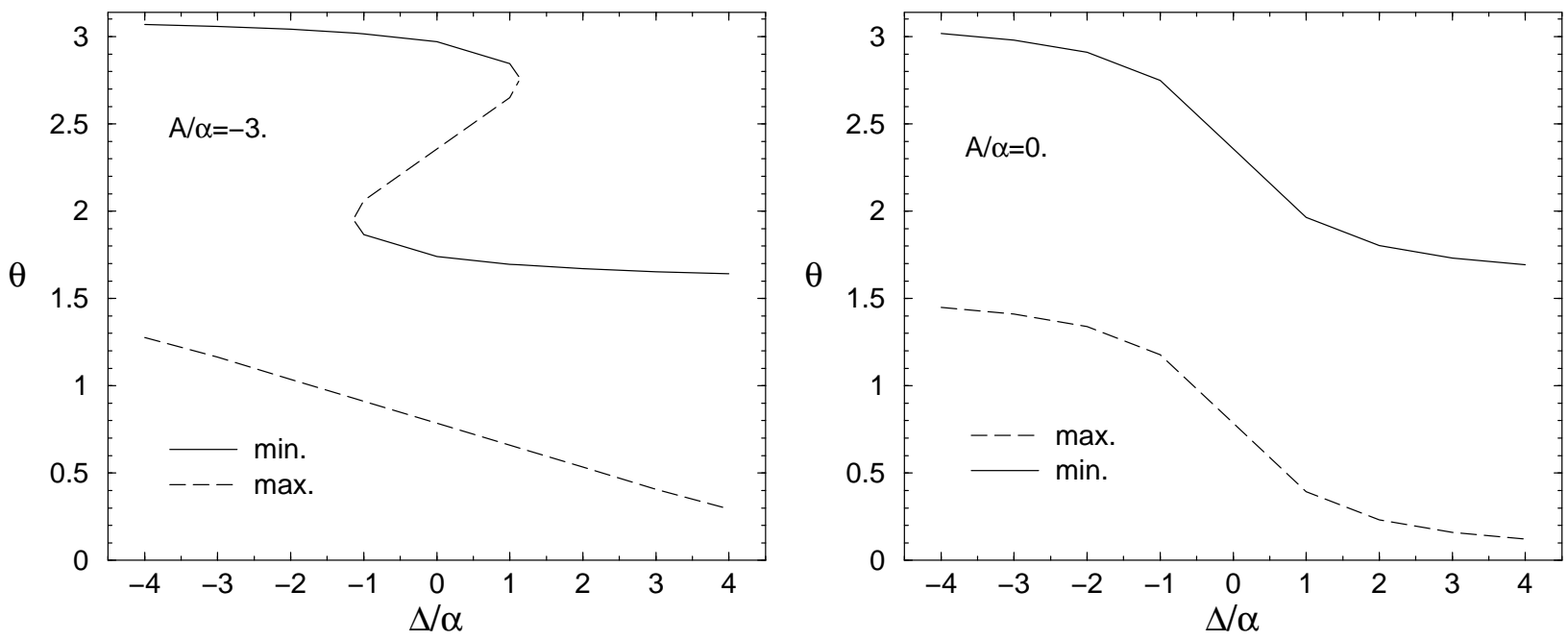

Figure 3.5. Internal mixing angle $\theta$ in the case of a uniform hybrid condensate as a function of $\Delta / \alpha$ for $A / \alpha=-3$ (left) and $A / \alpha=0$ (right). The upper curves on both graphs correspond to $\varphi=\pi$, while $\varphi=0$ in the lower curves. Full and dashed lines correspond respectively to minima and maxima of the energy functional.

$\frac{\langle H\rangle}{N}=\frac{\epsilon_{1}+\epsilon_{2}}{2}+C+\frac{A}{2} \cos ^{2} 2 \theta+\Delta \cos 2 \theta+\alpha \sin 2 \theta \cos \varphi$

where $\varphi=\varphi_{2}-\varphi_{1}$ is the relative phase between the two species and the newly defined dimensionless coefficients $C$, $A$ and $\Delta$ are

$$
\begin{array}{rlr}
C & =\frac{4 \pi \hbar^{2} N}{8 M \mathcal{V}}\left(a_{1}+a_{2}+2 a_{12}\right), & \\
A & =\frac{4 \pi \hbar^{2} N}{4 M \mathcal{V}}\left(a_{1}+a_{2}-2 a_{12}\right) & \text { and } \\
\Delta & =\frac{\delta}{2}+\frac{4 \pi \hbar^{2} N}{4 M \mathcal{V}}\left(a_{1}-a_{2}\right), &
\end{array}
$$

where $\delta=\epsilon_{1}-\epsilon_{2}$ is the detuning parameter.

Extrema of this function with respect to the relative phase $\varphi$ will exist when $\sin \varphi=0$, which implies $\varphi=0$ or $\varphi=\pi$. The extrema with respect to the mixing angle $\theta$ are easily determined for given values of the parameters from the stationarity condition

$\frac{1}{N} \frac{\partial\langle H\rangle}{\partial \theta}=0 \Longrightarrow \frac{A}{2 \alpha} \sin 4 \theta+\frac{\Delta}{\alpha} \sin 2 \theta+\cos 2 \theta \cos \varphi=0$.

Note that only two independent combinations of the various parameters appear in this equation. Some solutions are shown in Fig. 3.5. For sufficiently negative values of $A / \alpha$ one has two distinct minima when $\Delta / \alpha$ is in a certain vicinity of zero.

An analysis of these solutions from the point of view of the coherent ansatz leads of course to similar results. It can be found in ref. [32].

\section{One step beyond the Gross- Pitaevski description}

Gross-Pitaevski (G.-P.) theory, including the effective twobody interaction based on the scattering length, draws its most fundamental (if not most important) support from the results of Lieb and Seiringer[22] concerning the G.-P. limit. This limit carries a strong statement bearing on the ground state of the many boson system (with purely repulsive twobody interactions!), which essentially declares its "manybody triviality" (absence of many-body correlations, see section 2.4). Therefore it is very uninformative on any features that involve such correlations in an essential way, ranging from ground state properties under conditions falling short of the G.-P. limit to the nature and spectrum of low lying excitations.

One decisive first step towards dealing with these matters was taken long ago by Bogoliubov[42], on the basis of an heuristic approach which appears adequate for dilute systems, although such systems were not experimentally available at the time. Its concepts were soon re-elaborated from alternate points of view with a rather strong but unfulfilled aim of obtaining results valid for at least not-so-dilute systems (see e.g. refs. [12]).

Some of the main approaches to such matters, within the particular and now experimentally rich domain of dilute systems, will be treated in the sections to follow.

\subsection{Bogoliubov's quasi-particles}

The problem treated by Bogoliubov consisted in determining the nature of the low lying excitations of a Bose-Einstein condensate, and in particular its dispersion equation in an extended, homogeneous system, which means the dependence of the excitation energies on their momentum. Of course the atom-atom interaction plays an essential role here. 
Although one can obtain the results due to Bogoliubov in a variety of different ways ${ }^{5}$, it will be useful to present it here in the light of being some sort of perturbative correction to the pure G.-P. effective mean field picture, as this will also make way for the developments to follow.

Consider then the effective hamiltonian (2.5) for the simplest case of a uniform system, i.e., with $V(\vec{r}) \equiv 0$. It is convenient here to use a momentum representation defined in terms of normalized plane waves satisfying periodic boundary conditions in a quantization volume $\mathcal{V}$. The momentum representation of the field operators is then given as

$$
a_{\vec{k}}^{\dagger}=\frac{1}{\sqrt{\mathcal{V}}} \int d^{3} r e^{i \vec{k} \cdot \vec{r}} \psi^{\dagger}(\vec{r})
$$

They satisfy usual boson commutation relations. In this representation the kinetic energy is diagonal and the hamiltonian is written as

$$
H_{\text {eff }}=\sum_{\vec{k}} \frac{\hbar^{2} k^{2}}{2 M} a_{\vec{k}}^{\dagger} a_{\vec{k}}+\frac{\lambda}{2 \mathcal{V}} \sum_{\vec{k}_{1}, \vec{k}_{2}, \vec{q}} a_{\vec{k}_{1}+\vec{q}}^{\dagger} a_{\vec{k}_{2}-\vec{q}}^{\dagger} a_{\vec{k}_{1}} a_{\vec{k}_{2}}
$$

The sum over three momenta in the two-body term takes momentum conservation explicitly into account and the explicit assumption is made that $\lambda>0$.

The minimizer of the G.-P. functional for this hamiltonian is just the constant zero-momentum plane wave $1 / \sqrt{\mathcal{V}}$.
When the density of the system is such that there are $N$ particles in the quantization volume $\mathcal{V}$, the effective mean field ground state is

$$
|\Phi\rangle=\frac{1}{\sqrt{N !}}\left(a_{0}^{\dagger}\right)^{N}|0\rangle .
$$

It is obvious, however, that such a state is not an eigenstate of $H_{\text {eff }}$ on account of the two-body term, which in particular contains a contribution $a_{\vec{q}}^{\dagger} a_{-\vec{q}}^{\dagger} a_{0} a_{0}$ which, when acting on $|\Phi\rangle$, will convert a pair of zero momentum bosons into two bosons with momenta $\pm \vec{q}$. The corresponding amplitude will moreover contain a bosonic enhancement factor of $\sqrt{N(N-1)}$. These terms represent propensities of the hamiltonian which are completely frustrated within the bounds of the G.-P. approximation, which only takes into account terms in which all the field operators are zero momentum operators.

Now observe that, due to momentum conservation, the number of zero momentum operators in any term of the effective hamiltonian can be zero, one, two or four. In view of the expected large population of the zero momentum state and its consequences in terms of bosonic enhancement factors, a less drastic truncation would result if the only terms left out would be those with at most one zero momentum operator, and hence with three or four nonzero momentum operators, corresponding to single-boson states expected to be weakly populated. This would leave us with the not-sotruncated hamiltonian

$$
\begin{aligned}
H_{\mathrm{eff}}^{\prime} & =\frac{\lambda}{2 \mathcal{V}} a_{0}^{\dagger} a_{0}^{\dagger} a_{0} a_{0}+\sum_{\vec{k} \neq 0}\left[\frac{\hbar^{2} k^{2}}{2 M} a_{\vec{k}}^{\dagger} a_{\vec{k}}+\frac{\lambda}{2 \mathcal{V}}\left(a_{\vec{k}}^{\dagger} a_{-\vec{k}}^{\dagger} a_{0} a_{0}+a_{0}^{\dagger} a_{0}^{\dagger} a_{-\vec{k}} a_{\vec{k}}+4 a_{\vec{k}}^{\dagger} a_{0}^{\dagger} a_{0} a_{\vec{k}}\right)\right]= \\
& \equiv H_{0}^{\prime}+H_{q}^{\prime} .
\end{aligned}
$$

The term $H_{0}^{\prime}$ acts only in the zero momentum sector, while the term $H_{q}^{\prime}$ is characterized by a quadratic dependence on non-zero momentum field operators.

This hamiltonian clearly conserves the total number of bosons (i.e., commutes with $\hat{N} \equiv \sum_{\vec{k}} a_{\vec{k}}^{\dagger} a_{\vec{k}}$ ), but of course not the number of bosons in the zero momentum state, or the number of bosons in non-zero momentum states when considered separately (i.e., does not commute with $\hat{N}_{0} \equiv a_{0}^{\dagger} a_{0}$ or with $\hat{N}-\hat{N}_{0}$ ). Therefore its ground state will be com- plicated by entangled quantum fluctuations of these partial numbers. The standard way to circumvent these complications is again to decorrelate by factorization the zero momentum and the nonzero momentum parts of the state vector at the expense of the conservation of the total number of bosons. Furthermore, in order to keep the density of the system under control, one constrains the average number of atoms in the quantization volume by means of the Lagrange multiplier $\mu$, and considers thus

$$
H_{\mathrm{eff}}^{\prime}-\mu \hat{N}=H_{0}^{\prime}-\mu \hat{N}_{0}+\sum_{\vec{k} \neq 0}\left[\left(\frac{\hbar^{2} k^{2}}{2 M}-\mu\right) a_{\vec{k}}^{\dagger} a_{\vec{k}}+\frac{\lambda}{2 \mathcal{V}}\left(a_{\vec{k}}^{\dagger} a_{-\vec{k}}^{\dagger} a_{0} a_{0}+a_{0}^{\dagger} a_{0}^{\dagger} a_{-\vec{k}} a_{\vec{k}}+4 a_{\vec{k}}^{\dagger} a_{0}^{\dagger} a_{0} a_{\vec{k}}\right)\right]
$$

\footnotetext{
${ }^{5}$ In particular, they may be obtained by linearization of the time-dependent Gross-Pitaevski equation (the one-component counterpart of eqs. (3.16)) in the small amplitude oscillatory regime around the stationary equilibrium solution, see e.g. Chapter 13 of ref. [10].
} 
One way to implement this factorization without imposing further ansatz restrictions on the non-zero momentum part, assume that the zero momentum part of the state vector is frozen in a coherent state

$$
|z\rangle=e^{z a_{0}^{\dagger}-z^{*} a_{0}}|0\rangle
$$

which will in particular produce the appropriate bosonic enhancement factors once the average number of atoms is properly adjusted. In the spirit of a perturbative treatment of the quadratic terms, the values of $z$ and and $\mu$ are determined by minimization of the zero momentum energy functional with the proper number constraint:

$$
\delta\left(\left\langle z\left|H_{0}^{\prime}-\mu \hat{N}_{0}\right| z\right\rangle\right)=\delta\left(\frac{\lambda}{2 \mathcal{V}}|z|^{4}-\mu|z|^{2}\right)=0
$$

$$
\left\langle z\left|\hat{N}_{0}\right| z\right\rangle=|z|^{2}=N
$$

Variation of the functional leads to the equation

$$
\left(\frac{\lambda}{\mathcal{V}}|z|^{2}-\mu\right) z=0 \quad \text { hence } \quad|z|^{2}=\frac{\mathcal{V}}{\lambda} \mu=N
$$

discarding the trivial solution $z=0$. Thus $z=\sqrt{N} e^{i \gamma}$ and $\mu=\lambda N / \mathcal{V}$.

The effective hamiltonian $h_{\text {eff }}$ extended to the non-zero momentum sector is next obtained as the partial expectation value of $H_{\text {eff }}^{\prime}-\mu \hat{N}$ in the state $\left|\sqrt{N} e^{i \gamma}\right\rangle$. The phase $\gamma$ is in fact of no consequence since it can be absorbed by redefining the phases of the $\vec{k} \neq 0$ states, and will accordingly be omitted in the following. One obtains

$$
\begin{aligned}
h_{\mathrm{eff}} & =\frac{\lambda N^{2}}{2 \mathcal{V}}+\sum_{\vec{k}}\left(\frac{\hbar^{2} k^{2}}{2 M}-\mu\right) a_{\vec{k}}^{\dagger} a_{\vec{k}}+\frac{\lambda N}{2 \mathcal{V}} \sum_{\vec{k} \neq 0}\left(a_{\vec{k}}^{\dagger} a_{-\vec{k}}^{\dagger}+a_{-\vec{k}} a_{\vec{k}}+4 a_{\vec{k}}^{\dagger} a_{\vec{k}}\right)= \\
& =\frac{\lambda N^{2}}{2 \mathcal{V}}+\sum_{\vec{k}} \frac{\hbar^{2} k^{2}}{2 M} a_{\vec{k}}^{\dagger} a_{\vec{k}}+\frac{\lambda N}{2 \mathcal{V}} \sum_{\vec{k} \neq 0}\left(a_{\vec{k}}^{\dagger} a_{-\vec{k}}^{\dagger}+a_{-\vec{k}} a_{\vec{k}}+2 a_{\vec{k}}^{\dagger} a_{\vec{k}}\right) .
\end{aligned}
$$

In the final expression the Lagrange multiplier $\mu$ was substituted by its value obtained in (4.2).

The now standard way to deal with such an hamiltonian involves "canonical transformations" of the field operators of the form

$$
\eta_{\vec{k}}=u_{k} a_{\vec{k}}+v_{k} a_{-\vec{k}}^{\dagger}, \quad u_{k}^{2}-v_{k}^{2}=1 .
$$

This type of transformation preserves the commutation relations and can be inverted, with the result

$$
a_{\vec{k}}=u_{k} \eta_{\vec{k}}-v_{k} \eta_{-\vec{k}}^{\dagger}
$$

Substitution in (4.3) yields a quadratic form in the operators $\eta_{\vec{k}}, \eta_{\vec{k}}^{\dagger}$. The transformation coefficients $u_{k}, v_{k}$ can then be determined so as to eliminate what Bogoliubov called the "dangerous terms", involving two creation or two anihilation operators. As a result of this the hamiltonian (4.3) is reduced to the form

$$
h_{\mathrm{eff}}=\frac{\lambda N^{2}}{2 \mathcal{V}}+\delta h_{\mathrm{eff}}^{(0)}+\sum_{\vec{k}} \hbar \omega_{k} \eta_{\vec{k}}^{\dagger} \eta_{\vec{k}}
$$

where $\delta h_{\mathrm{eff}}^{(0)}$ is a c-number term resulting from normal ordering the "non-dangerous" terms. Thus the net result of this procedure is i) to generate a set of normal mode-like excitations with energy $\hbar \omega_{k}$ and momentum $\vec{k}$ created by the operators $\eta_{\vec{k}}^{\dagger}$; these are the Bogoliubov "quasi-particles", and ii) to give a correction $\delta h_{\text {eff }}^{(0)}$ to the G.-P. ground state energy. Note that this ground state must now be taken as the "quasiparticle vacuum", defined as the state which is annihilated by any of the operators $\eta_{\vec{k}}$.

\subsubsection{Easy route to the quasi-particles}

The procedure described above to obtain explicit expressions for the transformation coefficients $u_{k}, v_{k}$ and hence also for the important quantities $\omega_{k}$ and $\delta h_{\text {eff }}^{(0)}$ can, of course, be followed "verbatim". It is not, however, the shortest, or the more elegant route. A better approach on both of these aspects[32] involves, as a first step, rewriting the hamiltonian (4.3) in terms of the coordinate-like operators $x_{\vec{k}}$ and momentum-like operators $p_{\vec{k}}$ defined as

$$
x_{\vec{k}} \equiv \frac{a_{-\vec{k}}+a_{\vec{k}}^{\dagger}}{\sqrt{2}}, \quad p_{\vec{k}} \equiv \frac{a_{\vec{k}}-a_{-\vec{k}}^{\dagger}}{i \sqrt{2}}, \quad\left[x_{\vec{k}}, p_{\vec{k}^{\prime}}\right]=i \delta_{\vec{k}, \vec{k}^{\prime}}
$$


These definitions are easily inverted, giving the momentum space field operators in terms of $x_{\vec{k}}$ and $p_{\vec{k}}$. Substitution in (4.3) then gives

$$
h_{\mathrm{eff}}=\frac{\lambda N^{2}}{2 \mathcal{V}}+\sum_{\vec{k} \neq 0}\left[\left(\frac{\hbar^{2} k^{2}}{4 M}+\frac{\lambda N}{\mathcal{V}}\right) x_{\vec{k}} x_{-\vec{k}}+\frac{\hbar^{2} k^{2}}{4 M} p_{\vec{k}} p_{-\vec{k}}\right]-\sum_{\vec{k} \neq 0}\left(\frac{\hbar^{2} k^{2}}{4 M}+\frac{\lambda N}{2 \mathcal{V}}\right)
$$

The last term is a divergent $c$-number sum over momenta which will contribute to $\delta h_{\mathrm{eff}}^{(0)}$ and will be dealt with below.

The appropriate canonical transformations to obtain the quasi-particle excitations are now simple scale transformations

$$
\left\{\begin{array} { l } 
{ x _ { \vec { k } } } \\
{ p _ { \vec { k } } }
\end{array} \longrightarrow \left\{\begin{array}{l}
\Gamma_{k} \bar{x}_{\vec{k}} \\
\bar{p}_{\vec{k}} / \Gamma_{k}
\end{array}\right.\right.
$$

Writing $h_{\text {eff }}$ in terms of $\bar{x}_{\vec{k}}$ and $\bar{p}_{\vec{k}}$, and choosing the scale factors $\Gamma_{k}$ so that the coefficients of the $\bar{x}$-and $\bar{p}$-dependent terms are equal, i.e.

$$
\left(\frac{\hbar^{2} k^{2}}{4 M}+\frac{\lambda N}{\mathcal{V}}\right) \Gamma_{k}^{2}=\frac{\hbar^{2} k^{2}}{4 M} \frac{1}{\Gamma_{k}^{2}} \quad \text { implying } \quad \Gamma_{k}=\left[\frac{\frac{\hbar^{2} k^{2}}{4 M}}{\frac{\hbar^{2} k^{2}}{4 M}+\frac{\lambda N}{\mathcal{V}}}\right]^{\frac{1}{4}}
$$

one is left with

$$
\begin{aligned}
h_{\mathrm{eff}} & =\frac{\lambda N^{2}}{2 \mathcal{V}}-\sum_{\vec{k} \neq 0}\left(\frac{\hbar^{2} k^{2}}{4 M}+\frac{\lambda N}{2 \mathcal{V}}\right)+\sum_{\vec{k}} \sqrt{\frac{\hbar^{2} k^{2}}{4 M}\left(\frac{\hbar^{2} k^{2}}{4 M}+\frac{\lambda N}{\mathcal{V}}\right)}\left(\bar{x}_{\vec{k}} \bar{x}_{-\vec{k}}+\bar{p}_{\vec{k}} \bar{p}_{-\vec{k}}\right)= \\
& =\frac{\lambda N^{2}}{2 \mathcal{V}}-\sum_{\vec{k} \neq 0}\left(\frac{\hbar^{2} k^{2}}{4 M}+\frac{\lambda N}{2 \mathcal{V}}\right)+\sum_{\vec{k}} \sqrt{\frac{\hbar^{2} k^{2}}{2 M}\left(\frac{\hbar^{2} k^{2}}{2 M}+\frac{2 \lambda N}{\mathcal{V}}\right)}\left(\eta_{\vec{k}}^{\dagger} \eta_{\vec{k}}+\frac{1}{2}\right)
\end{aligned}
$$

where the quasi-particle operators are related to the scaled coordinate-like and momentum-like operators in the same way as the $a_{\vec{k}}, a_{\vec{k}}^{\dagger}$ are related to the $x_{\vec{k}}, p_{\vec{k}}$ :

$$
\bar{x}_{\vec{k}} \equiv \frac{\eta_{-\vec{k}}+\eta_{\vec{k}}^{\dagger}}{\sqrt{2}}, \quad \bar{p}_{\vec{k}} \equiv \frac{\eta_{\vec{k}}-\eta_{-\vec{k}}^{\dagger}}{i \sqrt{2}}, \quad\left[\bar{x}_{\vec{k}}, \bar{p}_{\vec{k}^{\prime}}\right]=i \delta_{\vec{k}, \vec{k}^{\prime}} .
$$

The transformation coefficients $u_{k}$ and $v_{k}$ relating the quasi-particle operators to the $a_{\vec{k}}, a_{\vec{k}}^{\dagger}$ are also easily obtained. In fact

$$
\eta_{\vec{k}}=\frac{\bar{x}_{-\vec{k}}+i \bar{p}_{\vec{k}}}{\sqrt{2}}=\frac{x_{-\vec{k}} / \Gamma_{k}+i \Gamma_{k} p_{\vec{k}}}{\sqrt{2}}=\frac{\left(1+\Gamma_{k}^{2}\right) a_{\vec{k}}+\left(1-\Gamma_{k}^{2}\right) a_{-\vec{k}}^{\dagger}}{2 \Gamma_{k}}
$$

from which it follows that

$$
u_{k}=\frac{1+\Gamma_{k}^{2}}{2 \Gamma_{k}} \quad \text { and } \quad v_{k}=\frac{1-\Gamma_{k}^{2}}{2 \Gamma_{k}} .
$$

We turn now to the effects of non-zero momentum states on the condensate dynamic properties. First, the quasiparticle energies are seen to be given by

$$
\hbar \omega_{k}=\sqrt{\frac{\hbar^{2} k^{2}}{2 M}\left(\frac{\hbar^{2} k^{2}}{2 M}+\frac{2 \lambda N}{\mathcal{V}}\right)} \quad \stackrel{k \rightarrow 0}{\longrightarrow} \sqrt{\frac{\lambda \rho_{p}}{M}} \hbar k .
$$

A remarkable outcome of this calculation is the phonon-like linear dependence with $\hbar k$ at low momenta. The slope is usually referred to as the sound velocity $c$, which can be expressed as

$$
c=\sqrt{\frac{\lambda \rho_{p}}{M}}=\frac{\hbar}{M} \sqrt{4 \pi \rho_{P} a}=\frac{\hbar}{M \xi_{H}}
$$

where $\xi_{H}$ is the healing length (2.14). The sound velocity is therefore a quantity which remains unchanged under the Gross-Pitaevski limit. The linear dispersion equation at small momenta has in fact been associated by Landau[43] with superfluidity of the condensate, as it prevents a particle moving in the condensate to lose energy by creating condensate excitations for phase-space reasons.

Second, collecting the various contributions to the correction to the ground state energy $\delta h_{\text {eff }}^{(0)}$, which includes now another divergent sum of zero-point-like energy terms coming from the quasi-particle hamiltonian, one is left with 


$$
\delta h_{\mathrm{eff}}^{(0)}=\frac{1}{2} \sum_{\vec{k} \neq 0}\left(\sqrt{\frac{\hbar^{2} k^{2}}{2 M}\left(\frac{\hbar^{2} k^{2}}{2 M}+\frac{2 \lambda N}{\mathcal{V}}\right)}-\frac{\hbar^{2} k^{2}}{2 M}-\frac{\lambda N}{\mathcal{V}}\right)
$$

still a divergent result! The divergence occurs for large momenta, as can be rewriting the summand (with obvious abbreviated notation) as

$$
\sqrt{e_{k}\left(e_{k}+2 \lambda \rho_{P}\right)}-e_{k}-\lambda \rho_{P}=e_{k}\left(\sqrt{1+\frac{2 \lambda \rho_{P}}{e_{k}}}-1-\frac{\lambda \rho_{P}}{e_{k}}\right) \stackrel{e_{k} \gg \lambda \rho_{P}}{\longrightarrow}-\frac{\lambda^{2} \rho_{P}^{2}}{2 e_{k}}+\frac{\lambda^{3} \rho_{P}^{3}}{2 e_{k}^{2}}+\cdots
$$

where the last result has been obtained by expanding the term with the square root. When the sum over $\vec{k}$ is transformed to an integral, the factor $k^{2}$ of the momentum space volume element will cancel the asymptotic $k^{-2}$ dependence of the first term, causing the integral to have a linear divergence proportional to $\lambda^{2}$. This divergence has been very specifically addressed in ref. [44], where it is shown that it results from the particular form of the two-body effective potential as implemented in the starting effective hamiltonian $^{6}$. This effective potential, written as

$$
v\left(\vec{r}_{1}, \vec{r}_{2}\right)=\lambda \delta\left(\vec{r}_{1}-\vec{r}_{2}\right)
$$

must be seen as a truncated version of the more accurate effective potential, introduced as the pseudopotential in ref.
[45],

$$
v\left(\vec{r}_{1}, \vec{r}_{2}\right)=\lambda \frac{\partial}{\partial\left|\vec{r}_{1}-\vec{r}_{2}\right|}\left[\left|\vec{r}_{1}-\vec{r}_{2}\right| \delta\left(\vec{r}_{1}-\vec{r}_{2}\right)\right] .
$$

These two forms of the potential give equivalent results $e x$ cept in order $\lambda^{2}$, where the effect of the more accurate version is simply to cancel out the second order term causing the divergence. In particular, the two forms of the effective potential are completely equivalent within the GrossPitaevski mean field approximation.

Introducing this amendment to the correction to the ground state energy, one is left with the now regular expression

$$
\delta h_{\mathrm{eff}}^{(0)}=\frac{1}{2} \sum_{\vec{k} \neq 0}\left(\sqrt{\frac{\hbar^{2} k^{2}}{2 M}\left(\frac{\hbar^{2} k^{2}}{2 M}+2 \lambda \rho_{P}\right)}-\frac{\hbar^{2} k^{2}}{2 M}-\lambda \rho_{P}+\frac{\lambda^{2} \rho_{P}^{2}}{2 e_{k}}\right)
$$

It can be calculated replacing the sum by an integral over momentum space[44] with the result

$$
\delta h_{\mathrm{eff}}^{(0)}=\frac{N \lambda \rho_{P}}{2} \frac{128}{15 \sqrt{\pi}} \sqrt{\rho_{P} a^{3}} .
$$

This result has been first derived by Lee and Yang in 1957[46] using a different method. It amounts only to a small correction in the dilute limit $\rho_{p} a^{3} \ll 1$ and in fact vanishes in the Gross-Pitaevski limit.

It is perhaps useful to summarize the main points of the preceding discussion, in which some effort was spent in trying to enhance the possibility of examination rather than belief. First, one takes into account off-diagonal momentum space matrix elements of the two-body effective interaction in which two of the four states involved are different from the macroscopically populated zero momentum state. This is considered as a perturbation of the condensed mean field ground state and determines a set of "normal excitation modes" which constitute the Bogoliubov quasi- particles. When the quantum fluctuations due to these excitation modes are taken into account by redefining the ground state as the "quasi-particle vacuum", an energy correction arises which is proportional to $\left(\rho_{P} a^{3}\right)^{1 / 2}$. In order to obtain this correction one must take special care in checking the consistency of the second order contributions of the two-body effective potential. The pseudopotential recipe of Huang and Yang[45] avoids the second order divergences in a way which is consistent with the kinship of the effective interaction with the two-body scattering amplitude.

\subsubsection{Ground state depletion}

The ground state state vector in the Bogoliubov approximation $\left|\Phi_{B}\right\rangle$ is the vacuum of the Bogoliubov quasi-particles and a coherent state of zero momentum single particle states, i.e.

\footnotetext{
${ }^{6} \mathrm{Cf}$. in this respect the treatment of the same problem in ref. [43]. Here the perturbative treatment of a two-body interaction potential is explicit, and the regularizing correction is obtained as the second order perturbative correction to the zero-momentum only ground state. Note that this point of view in untenable when the effective two-body interaction is related to the two-body scattering amplitude, since in this case the second order term is spurious.
} 


$$
\eta_{\vec{k}}\left|\Phi_{B}\right\rangle=0, \text { all } \vec{k} ; \quad a_{0}\left|\Phi_{B}\right\rangle=\sqrt{N}\left|\Phi_{B}\right\rangle
$$

The first of these properties implies that there are particles present in non-zero momentum states, since the quasiparticle operators $\eta_{\vec{k}}$ are linear combinations of creation and annihilation operators. One of the byproducts of the above calculation is therefore a perturbative estimate of particle number distribution in non-zero momentum states due to the action of the effective two-body interaction. This distribution is usually called the depletion of the condensate, from the fact that particles found in these states must have been removed from the zero momentum condensate. Strictly speaking, violation of the conservation of particle number in this calculation does not conform this meaning, however. Actually the depletion is here an additional distribution of particles which can be simply calculated from the expression which gives the number of particles with momentum $\vec{k} \neq 0$ in the quasi-particle vacuum, namely

$$
n_{\vec{k}} \equiv\left\langle\Phi_{B}\left|a_{\vec{k}}^{\dagger} a_{\vec{k}}\right| \Phi_{B}\right\rangle=v_{k}^{2}=\frac{\left(1-\Gamma_{k}^{2}\right)^{2}}{4 \Gamma_{k}^{2}}=\frac{\frac{\hbar^{2} k^{2}}{2 M}+\lambda \rho_{P}}{\sqrt{\frac{\hbar^{2} k^{2}}{2 M}\left(\frac{\hbar^{2} k^{2}}{2 M}+2 \lambda \rho_{P}\right)}}-1
$$

where use has been made of the previously obtained expression for $\Gamma_{k}$. Note that this expression refers to a given vector momentum, even though it depends only on it magnitude $k$. It diverges as $k^{-1}$ for small $k$. Due to this isotropy one can define a depletion density per interval of $k$ as

$$
\frac{d n}{d k} \equiv \frac{\mathcal{V}}{\left(2 \pi^{3}\right)} 4 \pi k^{2} n_{\vec{k}}
$$

in which the divergence for small $k$ has been controlled by the momentum space volume element. It is also easy to check that this depletion density approaches zero fast enough for large $k$ so that its integral, which corresponds to the "total depletion" in the Bogoliubov ground state is finite and also given in terms of the diluteness factor of the condensate as

$$
n_{\mathrm{tot}}=\frac{8 N}{3 \sqrt{\pi}}\left(\rho_{P} a^{3}\right)^{1 / 2} .
$$

Note that this expression also vanishes in the GrossPitaevski limit, in which $\rho_{P} a^{3} \rightarrow 0$.

\subsection{Self-consistency looks worse}

A crucial element in the Bogoliubov approach is the canonical transformation leading from the particle field operators to the quasi-particle operators, which is there used to obtain normal-mode-like excitations with non-zero momentum added perturbativelu on top of a zero-momentum condensate. An improvement over the perturbative character of this approach consists in starting from a slightly more general canonical transformation which will allow both for a coherent condensate and for quasiparticles and to determine both ingredients self-consistently. This is, in words, what is known as the Hartree-Fock-Bogoliubov (HFB) approximation, which we proceed to develop in continuation[47, 48].

The dynamics is based again on the hamiltonian (4.1), the system being assumed to be uniform and treated in a momentum representation defined in terms of periodical boundary conditions in quantization volume $\mathcal{V}$. The ground state will be approximated by a "self-consistent vacuum" of quasi-particle (normal mode-like) excitations associated to creation and annihilation operators $\eta_{\vec{k}}^{\dagger}, \eta_{\vec{k}}$ related to the corresponding momentum space components $a_{\vec{k}}^{\dagger}, a_{\vec{k}}$ of the field operator through the canonical transformation

$$
a_{\vec{k}} \equiv c_{\vec{k}}+z_{0} \delta_{\vec{k}, 0} \equiv\left(u_{k} \eta_{\vec{k}}-v_{k} \eta_{-\vec{k}}^{\dagger}\right)+z_{0} \delta_{\vec{k}, 0}
$$

where $z_{0}$ is a $c$-number and $u_{k}^{2}-v_{k}^{2}=1$. For $\vec{k} \neq 0$ this is of course just the Bogoliubov transformation leading to the quasi-particle operators, but for $\vec{k}=0$ the possibility of a $c$-number displacement of the particle operator is included through the parameter $z_{0}$. The interpretation of this parameter follows from the fact that the state vector $|\tilde{0}\rangle$ which is annihilated by the displaced operator $c_{0}$ has the property

$$
c_{0}|\tilde{0}\rangle=0 \Longrightarrow\left(a_{0}-z_{0}\right)|\tilde{0}\rangle=0 \Longrightarrow a_{0}|\tilde{0}\rangle=z_{0}|\tilde{0}\rangle,
$$

i.e., it is the eigenstate of the annihilation operator $a_{0}$ with eigenvalue $z_{0}$ and hence ( up to an overall phase) the coherent state

$$
|\tilde{0}\rangle \equiv\left|z_{0}\right\rangle \equiv e^{z_{0} a_{0}^{\dagger}-z_{0}^{*} a_{0}}|0\rangle .
$$

Note that one could have performed the Bogoliubov transformation before introducing the zero momentum $c$-number displacement, with equivalent results. In fact, in this case one would first have written

$$
a_{\vec{k}}=u_{v} \tilde{\eta}_{\vec{k}}-v_{k} \tilde{\eta}_{-\vec{k}}^{\dagger}
$$

and then introduce the zero momentum displacement by defining

$$
\tilde{\eta}_{\vec{k}}=\eta_{\vec{k}}+\zeta_{0} \delta_{\vec{k}, 0} .
$$

Combining these two steps one is left with

$$
a_{\vec{k}}=u_{k} \eta_{\vec{k}}-v_{k} \eta_{-\vec{k}}^{\dagger}+\left(u_{0} \zeta_{0}-v_{0} \zeta_{0}^{*}\right) \delta_{\vec{k}, 0}
$$


which is equivalent to (4.4) and establishes the relation of $z_{0}$ to $\zeta_{0}$.

The canonical transformation (4.4) therefore makes all the ingredients used in the Bogoliubov treatment again fully available. However, this transformation will now be used in a way that attempts to determine its parameters in a simultaneous, "self-consistent" way instead of in the sequential way used in that treatment.

The first step is straightforward, if algebraically rather cumbersome. In consists in substituting the transformed operators (4.4) into the effective hamiltonian, normal-ordering the quasiparticle operators and sorting the resulting terms according to the number of normal ordered quasiparticle operators they contain. In order to enforce the appropriate density (average number of particles $N$ in the quantization volume $\mathcal{V}$ ) a Lagrange multiplier $\mu$ is introduced. Again one finds that the phase of the zero momentum displacement $z_{0}$ can be absorbed by redefining the phases of the $k \neq 0$ single-particle states, so that this quantity will simply be taken as real in what follows. One obtains in this way terms having zero to four quasiparticle operators, collected respectively as $h_{i}$, with $i$ ranging from zero to four. Thus

$$
H_{\text {eff }}-\mu \sum_{\vec{k}} a_{\vec{k}}^{\dagger} a_{\vec{k}} \longrightarrow \sum_{i=0}^{4} h_{i} .
$$

Now if $|\Phi\rangle$ is the (normalized) quasi-particle vacuum, i.e. $\eta_{\vec{k}}|\Phi\rangle=0$ for all $\vec{k}$, then the expectation value of $H_{\text {eff }}-\mu \hat{N}$ in this state reduces to the $c$-number part $h_{0}$, which is given by

$$
h_{0}=-z_{0}^{2} \mu+\frac{\lambda}{2 \mathcal{V}} z_{0}^{4}+\sum_{\vec{k}}\left(e_{k}-\mu+2 \lambda z_{0}^{2}\right) v_{\vec{k}}^{2}-\frac{\lambda}{\mathcal{V}} z_{0}^{2} \sum_{\vec{k}} u_{\vec{k}} v_{\vec{k}}+\frac{\lambda}{\mathcal{V}}\left[\left(\sum_{\vec{k}} v_{\vec{k}}^{2}\right)^{2}+\frac{1}{2}\left(\sum_{\vec{k}} u_{\vec{k}} v_{\vec{k}}\right)^{2}\right]
$$

where $e_{k} \equiv \hbar^{2} k^{2} / 2 M$. Note that there is no guarantee that the sums over momenta which appear in this expression are finite. Ignoring this question for the moment, and proceeding to the formal minimization of this quantity with respect to the parameters of the canonical transformations, leads to the equations

$$
\begin{aligned}
& \frac{\partial h_{0}}{\partial z_{0}}=0 \Rightarrow z_{0}\left[-\mu+\frac{\lambda}{\mathcal{V}}\left(z_{0}^{2}+\sum_{\vec{k}}\left(2 v_{k}^{2}-u_{k} v_{k}\right)\right)\right]=0 \\
& \frac{\partial h_{0}}{\partial v_{k}}+\frac{\partial h_{0}}{\partial u_{k}} \frac{\partial u_{k}}{\partial v_{k}}=0 \Rightarrow \tanh 2 \sigma_{k}=\frac{\frac{\lambda}{\mathcal{V}}\left(z_{0}^{2}-\sum_{k^{\prime}} u_{k^{\prime}} v_{k^{\prime}}\right)}{e_{k}+2 \frac{\lambda}{\mathcal{V}}\left(z_{0}^{2}+\sum_{\vec{k}^{\prime}} v_{k^{\prime}}^{2}\right)-\mu},
\end{aligned}
$$

where the $u_{\vec{k}}$ and $v_{\vec{k}}$ have been parametrized in terms of the hyperbolic angle $\sigma_{k}$ as

$$
u_{k}=\cosh \sigma_{k}, \quad \text { and } \quad v_{k}=\sinh \sigma_{k}
$$

so as to take into account the condition $u_{k}^{2}-v_{k}^{2}=1$. The subsidiary condition on the number of particles, on the other hand, gives the additional equation

$$
\langle\Phi|\hat{N}| \Phi\rangle=N \Rightarrow z_{0}^{2}+\sum_{\vec{k}} v_{k}^{2}=N
$$

The interesting remaining part is of course the "nondangerous" part of $h_{2}$, which reads

$$
h_{2}=\sum_{\vec{k}} \hbar \omega_{k} \eta_{\vec{k}}^{\dagger} \eta_{\vec{k}}, \quad \hbar \omega_{k}=\sqrt{\left[e_{k}+\frac{\lambda}{\mathcal{V}}\left(z_{0}^{2}+\sum_{\vec{k}^{\prime}} u_{k^{\prime}} v_{k^{\prime}}\right)\right]^{2}-\frac{\lambda^{2}}{\mathcal{V}^{2}}\left(z_{0}^{2}-\sum_{\vec{k}^{\prime}} u_{k^{\prime}} v_{k^{\prime}}\right)^{2}} .
$$


Note that

$$
\hbar \omega_{k} \stackrel{k \rightarrow 0}{\longrightarrow} \sqrt{4 \frac{\lambda^{2}}{\mathcal{V}^{2}} z_{0}^{2} \sum_{\vec{k}^{\prime}} u_{k^{\prime}} v_{k^{\prime}}} \equiv \Delta_{\mathrm{HFB}}
$$

so that the dispersion equation for the quasiparticles of the HFB approximation does not have the interesting phonon behavior of the simple Bogoliubov approximation at small momenta. The quantity $\Delta_{\mathrm{HFB}}$ is usually referred to as the "HFB energy gap". One of the factors contributing to this energy gap is the sum which can be recognized as giving the contribution of the non condensate particles to the so called pairing (or anomalous) density associated with the quasiparticle vacuum

$$
\rho_{\vec{k}}^{(\text {pair })} \equiv\left\langle\Phi\left|a_{\vec{k}} a_{-\vec{k}}\right| \Phi\right\rangle=z_{0}^{2} \delta_{\vec{k}, 0}-u_{k} v_{k}
$$

The "normal" density can also be split into condensate and non-condensate contributions as

$$
\rho_{\vec{k}}^{\text {(norm) }} \equiv\left\langle\Phi\left|a_{\vec{k}}^{\dagger} a_{\vec{k}}\right| \Phi\right\rangle=z_{0}^{2} \delta_{\vec{k}, 0}+v_{k}^{2} .
$$

A "gapless" approximation can therefore be obtained from the HFB approximation by simply ignoring the contribution of the non-condensate particles to the pairing density. This approximation is known as the Popov approximation[47, 49].

\subsubsection{Difficulties with the contact effective interaction}

It has been mentioned that the results obtained for the HFB approximation were formal in the sense that the convergence of the sums over momenta was not being checked explicitly. It so happens that, for the case of the contact effective two body interaction included in the hamiltonian (4.1), the sum over the anomalous density contributions that are ignored in the Popov approximation are not convergent. This may be seen as follows. First, assume that the sum for the anomalous density converges. If so, then the second of eqs. (4.6) shows that for sufficiently large $k$ the hyperbolic angle $\sigma_{k}$, and hence also its hyperbolic sine which corresponds to $v_{k}$ decrease as $k^{-2}$. The sum is however to be taken over all directions of the momenta as well, and this contributes a factor $k^{2}$ from the volume element in momentum space. Since $u_{k}$ approaches one from above, for large $k$, it follows that the sum in fact cannot converge.

One easy way to avoid this divergence [48] is to replace the contact effective interaction by a finite range one, such as

$$
V\left(\vec{r}_{1}-\vec{r}_{2}\right) \longrightarrow \frac{4 \pi \hbar^{2} a}{M}\left(\frac{1}{\sqrt{\pi} b}\right)^{3} e^{-\frac{\left|\vec{r}_{1}-\vec{r}_{2}\right|^{2}}{b^{2}}}
$$

which reproduces the usual form of the contact interaction in the limit $b \rightarrow 0$. The two-body matrix elements of this interaction in momentum space involve

$$
V(q)=\frac{1}{\mathcal{V}} \int d^{3} r e^{i \vec{q} \cdot \vec{r}} V(\vec{r})=\frac{4 \pi \hbar^{2} a}{M \mathcal{V}} e^{-\frac{b^{2} q^{2}}{4}} \quad \stackrel{b \rightarrow 0}{\longrightarrow} \quad \frac{4 \pi \hbar^{2} a}{M \mathcal{V}}=\frac{\lambda}{\mathcal{V}}
$$

The two-body term of the effective hamiltonian (4.1) becomes

$$
\sum_{\vec{k}_{1}, \vec{k}_{2}, \vec{q}} V(q) a_{\vec{k}_{1}-\vec{q}}^{\dagger} a_{\vec{k}_{2}+\vec{q}}^{\dagger} a_{\vec{k}_{2}} a_{\vec{k}_{1}}
$$

and the paring density contributions e.g. to the second eq. (4.6) appear in the form

$$
\tanh 2 \sigma_{k}=\frac{\left(z_{0}^{2}-\sum_{k^{\prime}} V\left(\left|\vec{k}-\vec{k}_{1}\right|\right) u_{k_{1}} v_{k_{1}}\right)}{e_{k}+\left((V(0)+V(k)) z_{0}^{2}+\sum_{\vec{k}_{1}}\left(V(0)+V\left(\left|\vec{k}-\vec{k}_{1}\right|\right)\right) v_{k_{1}}^{2}\right)-\mu},
$$

the divergence being now controlled by the momentum transfer dependence of the two-body matrix elements. With this choice of effective two body interaction the HFB approximation is finite but still features a gap in the quasiparticle dispersion equation.

This approach certainly produces a calculable selfconsistent mean field theory on which one can, in particular, mount further improvements, by considering small amplitude fluctuations around the mean field. This has been pursued in ref. [48] and will be briefly reviewed in the next section. What remains in relatively less secure grounds is its relation to the actual physical situation, since the finite range of the potential, which is the ingredient which is essential to make the theory finite, is in fact not tied to it in any reasonably secure manner. Recall, furthermore, that the adopted zero momentum fit to the usual function of the scattering length makes the finite range potential conceptually akin to the scattering amplitude associated with the realistic atom-atom interaction.

One question which can be put at this point is whether the full recipe of Huang and Yang[46] for the pseudopotential is useful in this connection. One way of approaching this 
question is to try and evaluate the ground state energy in the HFB approximation, which means to evaluate the $c$-number term $h_{0}$ of the hamiltonian expressed in terms of the shift parameter $z_{0}$ and of normal ordered products of quasiparticle operators. Self-consistency makes this task much more demanding than the corresponding one in the simple Bogoliubov approximation, however, since the values of the various parameters appearing in $h_{0}$ are actually determined by the extremum conditions (4.6) derived from $h_{0}$ itself. There seems to be no calculation done along these lines in terms of the full pseudopotential, and there possibly will never be one.

\subsection{Elementary excitations on worse looking richer foundations}

Static solutions to self-consistent mean field approximations such as Hartree-Fock and Hartree-Fock-Bogoliubov are interesting starting points for studying stability and normal modes of excitation. One of the interesting and often explored features of such theories is that continuous symmetries of the hamiltonian which are broken in the mean field solution reappear as solutions of zero frequency, associated with the generators of the broken symmetry transformations, in the so called Random Phase Approximation (RPA) treatment of the excitation modes. The symmetry breaking itself can be seen as the resource explored by the mean field treatment to take important correlations into account within the limitations imposed by the mean field constraint.

Possibly the example of this syndrome that is most simply visualized is the generation of the self-bound character of atomic nuclei within the Hartree-Fock approximation. The finiteness of the bound nuclear system is clearly a correlation property, each nucleon being correlated so as not to be too far away from other nucleons, their center of mass behaving as a free particle with the appropriate total mass. This correlation property is replaced in the self-consistent mean field treatment by an attractive average potential generated by all nucleons and also binding them. In this way the mean field treatment replaces "not being too far from the rest" simply by "being confined by the common average potential", keeping all the nucleons together without they having to keep track of each other. This is achieved, however, at the expense of breaking the translational symmetry of the hamiltonian through the introduction of the average localizing potential. One of the solutions to the RPA equations to identify excitation modes of such a system will be the total momentum operator, the generator of spatial translations of the system, with excitation frequency equal to zero, implying the absence of a restoring force as a consequence of the symmetry (see e.g. ref. [50], section 8.4.7).

In the context of the Bose-Einstein condensates these features remain true and can be taken advantage of when one uses as a starting point to study excitation modes the self-consistent HBF approximation. Although the relation of calculated results to the actual physical situation involves the question of the effective interaction which is used, general properties which are not oversensitive to it, such as the fate and role played by the energy gap in the BHF spectrum as well as the fate and role of the broken symmetries, can be safely investigated using self-consistently a finite range potential such as the one introduced in the preceding section.

A possible starting point for this brief review of the main results of ref. [48] is the observation that the generator of the symmetry which is broken by the HFB treatment is the number operator $\hat{N}=\sum_{\vec{k}} a_{\vec{k}}^{\dagger} a_{\vec{k}}$, which can be written in terms of the quasiparticle operators and the $c$-number displacement $z_{0}$ as

$$
\hat{N}=z_{0}^{2}+\sum_{\vec{k}} v_{k}^{2}+z_{0}\left(u_{0}-v_{0}\right)\left(\eta_{0}+\eta_{0}^{\dagger}\right)-\sum_{\vec{k} \geq 0} \frac{2 u_{k} v_{k}}{1+\delta_{\vec{k}, 0}}\left(\eta_{\vec{k}}^{\dagger} \eta_{-\vec{k}}^{\dagger}+\eta_{\vec{k}} \eta_{-\vec{k}}\right)+\sum_{\vec{k}}\left(u_{k}^{2}+v_{k}^{2}\right) \eta_{\vec{k}}^{\dagger} \eta_{\vec{k}}
$$

where the restriction on the second sum means that each pair of opposite vectors $\vec{k},-\vec{k}$ in to be included only once. The first two terms are the only ones to survive when the expectation value is taken in the quasiparticle vacuum $|\Phi\rangle$, and must therefore give the number of particles $N$. The operator part should emerge as a zero frequency normal mode in the desired RPA treatment, and thus its form serves to indicate the minimal structure needed for the excitation operators to be determined. The quasi-particle representation of $\hat{N}$ shows that besides quasiparticle creation and annihilation operators, two-quasiparticle operator terms also appear. It turns out that contributions of the form $\eta_{\vec{k}}^{\dagger} \eta_{\vec{k}^{\prime}}$ are not relevant for the equations of motion to be derived for the excitation operators, while it is important to include the quasiparticle creation and anihilation terms. Thus the general ansatz for the elementary excitation operator with momentum $\vec{P}$ is taken to be

$$
Q_{\vec{P}}^{\dagger}=x_{\vec{P}} \eta_{\vec{P}}^{\dagger}+y_{\vec{P}} \eta_{-\vec{P}}+\sum_{\vec{q} \geq 0} X_{\vec{q}, \vec{P}} \frac{\eta_{\vec{q}+\vec{P} / 2}^{\dagger} \eta_{-\vec{q}+\vec{P} / 2}^{\dagger}}{\sqrt{1+\delta \vec{q}, 0}}+Y_{\vec{q}, \vec{P}} \frac{\eta_{\vec{q}-\vec{P} / 2} \eta_{-\vec{q}-\vec{P} / 2}}{\sqrt{1+\delta \vec{q}, 0}}
$$



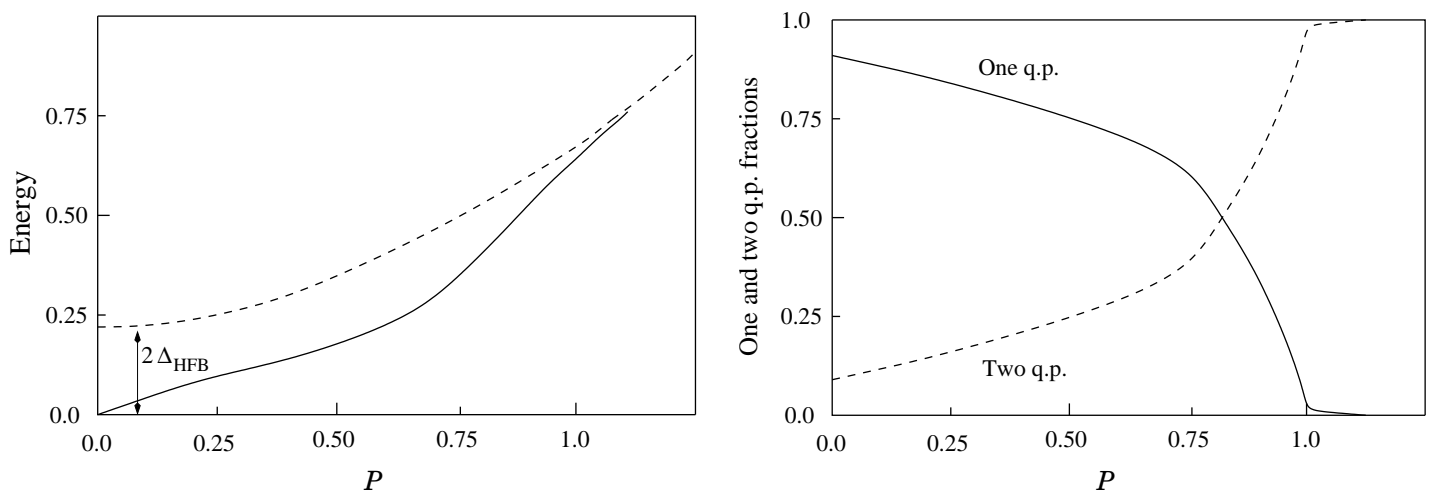

Figure 4.1. Left: Numerical dispersion equation for RPA elementary excitations on the HFB quasiparticle vacuum. The calculation has been done assuming a not so dilute system with $\rho a^{3}=.01$. The momentum scale is plotted in units of $\hbar / a$, and the energy scale is in units of $\hbar^{2} / 2 M a^{2}$. The full line represents a discrete phonon-like branch, and the dashed line represents the threshold of a continuum. At zero momentum $P$ the threshold is at twice the HFB gap. Note that the discrete branch essentially merges with the continuum at $P \sim \hbar / a$. Right: One and two quasi-particle fractions in the composition of the discrete branch. The shift from one quasi-particle to two-quasiparticle dominance at $P \sim 0.75 \hbar / a$ suggests an "avoided crossing" situation, with most of the one quasi-particle strength located within the continuum branch for higher momenta.

where the $x_{\vec{P}}, y_{\vec{P}}$ and $X_{\vec{q}, \vec{P}}, Y_{\vec{q}, \vec{P}}$ are coefficients to be determined. These two groups of coefficients, which refer to one and two quasi-particle components of the sought normal modes (three, or more, quasiparticle contributions to the excitation operator are ignored), are coupled to each other through the three quasiparticle part $h_{3}$ of the decomposition (4.5) of the effective hamiltonian. In addition the four quasiparticle term $h_{4}$ couples the two quasi-particle components among themselves. Note that all terms create a momentum $\vec{P}$, either through the addition or removal of one quasiparticle or through the addition or removal of a pair of quasipar- ticles with relative momentum $\vec{q}$.

The total momentum $\vec{P}$ of an excitation mode is a sharply defined quantity, so that the RPA equations determining the coefficients $x_{\vec{P}}, y_{\vec{P}}$ and $X_{\vec{q}, \vec{P}}, Y_{\vec{q}, \vec{P}}$ have to be solved for each value of $\vec{P}$ (or of just the magnitude $P$, due to rotational invariance). Implementing the equations in terms or periodical boundary conditions in a quantization volume $\mathcal{V}$, reduces them to large matrix equations which are however amenable to numerical solution. They yield, for each $P$, a set of modes $Q_{\vec{P}}^{\sigma \dagger}$ whose associated coefficients are chosen to satisfy the normalization condition

$$
\left\langle\Phi\left|\left[Q_{\vec{P}}^{\tau}, Q_{\vec{P}}^{\sigma \dagger}\right]\right| \Phi\right\rangle=x_{\vec{P}}^{\tau *} x_{\vec{P}}^{\sigma}-y_{\vec{P}}^{\tau *} y_{\vec{P}}^{\sigma}+\sum_{\vec{q} \geq 0}\left(X_{\vec{q}, \vec{P}}^{\tau *} X_{\vec{q}, \vec{P}}^{\sigma}-Y_{\vec{q}, \vec{P}}^{\tau *} Y_{\vec{q}, \vec{P}}^{\sigma}\right)=\delta_{\tau \sigma}
$$

Solutions obtained for a gaussian effective two-body interaction of the form (4.7) with $b=3,96 a$ and assuming that the diluteness parameter $\rho a^{3}$ is .01, are summarized in Fig. 4.1. What one obtains for each value of $P$ is a discrete, lower energy solution in addition to a "continuum" (within the limitations of the scheme based on the adoption of periodical boundary conditions) of solutions starting at a $P$ dependent threshold energy which goes to the limit of twice the HFB gap for $P \rightarrow 0$. The set of discrete solutions form a low energy discrete branch of excitations with phonon behavior, and merge at $P=0$ with the the symmetry generator $\hat{N}$.

Reference to the dependence of the coefficients $x_{\vec{P}}, y_{\vec{P}}$ and $X_{\vec{q}, \vec{P}}, Y_{\vec{q}, \vec{P}}$ on the total momentum $\vec{P}$ gives additional information on the composition of the normal modes. As shown on the right hand graph of Fig. 4.1, for low momenta the discrete branch is dominated by the one quasi-particle contributions

$$
c_{1}(\vec{P})=\left|x_{\vec{P}}\right|^{2}-\left|y_{\vec{P}}\right|^{2}
$$

while the contributions of two quasi-particle terms

$$
c_{2}(\vec{P})=\sum_{\vec{q} \geq 0}\left(\left|X_{\vec{q}, \vec{P}}\right|^{2}-\left|Y_{\vec{q}, \vec{P}}\right|^{2}\right)=1-c_{1}(\vec{P})
$$

remain small. This dominance of one quasi-particle terms decreases as $P$ increases and is replaced by a two quasiparticle dominance near the $P$ values where the energy of the discrete branch approaches the continuum threshold and eventually merges with it.

This behavior suggests an "avoided crossing" situation, as the one free HFB quasi-particle energy and the threshold for two free quasi-particles cross. At values of $P$ beyond the onset of the two quasi-particle dominance in the discrete branch, the bulk of the one quasi-particle strength is to be found spread in the continuum branch. 


\section{Many mode traps, localization and interference}

As discussed in Chapter 2, the state of a dilute system of very cold trapped bosons (in the sense that the system finds itself esentially in its ground-state) can, to a very good approximation, be described in terms of a single onebody wavefunction, which minimizes the appropriate GrossPitaevski functional. In particular, this result becomes rigorously valid in the Gross-Pitaevski limit (see section 2.4), independently of possibly ellaborate varieties of trapping potentials, provided they satisfy the general requirements of local regularity and confinement $\left(\lim _{r \rightarrow \infty} V(\vec{r})=\infty\right.$, see ref. [15]). Some potentials, such as "many wells" potentials can lead to nearly degenerate solutions, so that restriction to the single minimizer state may not be physically appropriate, as it artificially freezes the possibly interesting dynamics which involves the quasi-degenerate group of modes. On the other hand, to the extent that one increases the relevant sector of the phase space by considering many, quasi-degenerate single-particle modes one also allows for a richer choice of observables, which may or may not be in fact brought to measurement procedures in the laboratory. One may therefore expect that closer scrutiny of the measurement processes also becomes more relevant in this context. This final chapter will be dedicated to "a bird's eye view" of these topics. Before turning to the modeling of many-mode setups, it is useful to review some typical experimental facts observed in connection with such situations.

\subsection{Quick survey of phenomena with some in- terpretation hints}

A most famous first observation is that of Andrews et al.[51], in which a sausage shaped condensate is pinched in the middle by a very high optical barrier, which essentially cuts it in two pieces. When these are allowed to expand by removal of the trap and barrier, they generate interference fringes visible under absorption imaging. This experiment has been carefully analysed in ref. [52], in terms of a time-dependent Gross-Pitaevski initial conditions problem in a way which is consistent with the use of a single, initially bi-localized wavefunction. The observed interference fringes can in this case be obtained from the one body density. Thus, if after expansion the time evolved wavefunction can be represented over some region as a superposition of two plane waves (the two different momenta resultung from contributions of the two initially disjoint parts to the amplitude in that region), then the density is there given by

$$
\phi(\vec{r}) \sim a e^{i \vec{k}_{1} \cdot \vec{r}}+b e^{i \vec{k}_{2} \cdot \vec{r}} \longrightarrow \rho(\vec{r})=|a|^{2}+|b|^{2}+2 \operatorname{Re}\left(a b^{*} e^{i\left(\vec{k}_{1}-\vec{k}_{2}\right) \cdot \vec{r}}\right)
$$

This is then simple one body interference, the many body nature of the condensate serving just obtaining the necessary measurement statistics in one shot[53].

A different type of interference effect which may occur in a many boson system was pointed out, even before the experiment of Andrews et al., by Javanainae and Yoo[54]. This type of interference does not appear in the one-body density and is basically a correlation effect, requiring the implementation of a many body observable for its direct measurement.
To illustrate it in the simplest possible way, consider the state of two free identical bosons

$$
\Phi\left(\vec{r}_{1}, \vec{r}_{2}\right)=\frac{1}{\sqrt{2} \mathcal{V}}\left(e^{i \vec{k}_{1} \cdot \vec{r}_{1}} e^{i \vec{k}_{2} \cdot \vec{r}_{2}}+e^{i \vec{k}_{1} \cdot \vec{r}_{2}} e^{i \vec{k}_{2} \cdot \vec{r}_{1}}\right)
$$

The full probability density associated with this exchange correlated wavefunction is

$$
\left|\Phi\left(\vec{r}_{1}, \vec{r}_{2}\right)\right|^{2}=\frac{1}{\mathcal{V}^{2}}\left(1+\cos \left(\vec{k}_{1}-\vec{k}_{2}\right) \cdot\left(\vec{r}_{1}-\vec{r}_{2}\right)\right)=\frac{2}{\mathcal{V}^{2}} \cos ^{2} \frac{\left(\vec{k}_{1}-\vec{k}_{2}\right) \cdot\left(\vec{r}_{1}-\vec{r}_{2}\right)}{2}
$$

which shows interference fringes in the relative position of the two bosons. Thus, interference fringes would be seen in the reiterated measurement of the relative position of the two bosons in identically prepared systems. At the same time, the one body density associated with the two boson wavefunction $\Phi\left(\vec{r}_{1}, \vec{r}_{2}\right)$ is easily seen to be constant, so that reiterating a one body position measurement yields a flat distribution. The point of Javanainen and Yoo in ref. [54] is that a similar situation ocurs when $N / 2$ particles occupy each one of two plane-wave states, in the sense that the measurement of $N-1$ conditional positions, each measurement being conditioned by all the preceding results shows interference fringes similar to those observed in the one-body density in the case of single wavefunction consisting of the superposition of two plane waves. A single full set of conditioned position measurements, for sufficiently large $N(N=1000$ 
in a simulation shown in ref. [54]) in fact exhibits the many body exchange correlation fringes clearly enough.

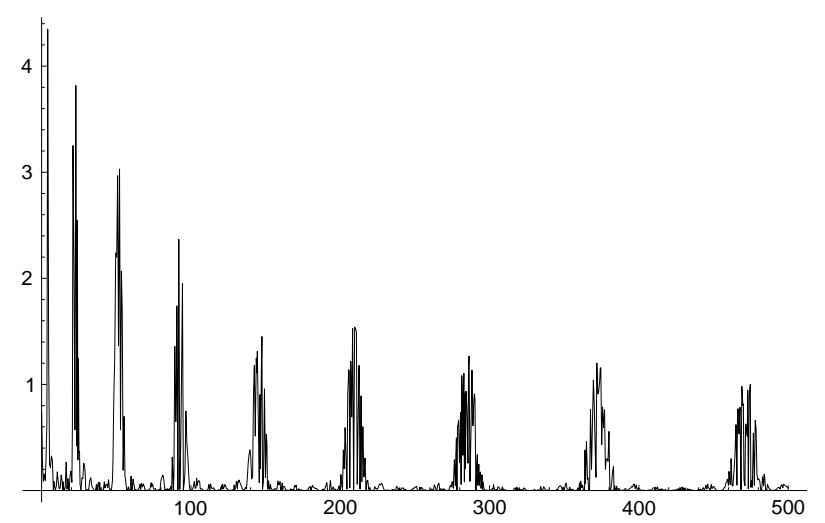

Figure 5.1. Intensity $I(q)$ (in arbitrary units) obtained from eq. (5.1) with $n=6$ and the realistic parameters for the Anderson and Kasevich experiment $q_{0}=.425 \mu m$ and $\frac{2}{3} \frac{M}{\hbar} \sqrt{2 g} q_{0}^{3 / 2}=1.130$. The horizontal scale is in $\mu \mathrm{m}$. Compare with Fig. 4 of ref. [56].

Recently, a related experiment has been reported by Shin et al.[55] involving however entirely different aspect-ratios, as in this case an elongated condensate was divided lengthwise into two also very elongated parts by a controlable barrier.

A different setup which again can be interpreted in terms of the dominance of a single, multiply split wavefunction occurs in the experiment of Anderson and Kasevich[56]. Here the vertically arranged $\sim 30$ portions of a multiply split condensate are allowed to leak, the various leakages suffering free falls from their respective initial positions. Under these conditions one observes at a given time the existence of zones of measurable density, which can be accurately reproduced in terms of constructive interference of $n$ free falling coherent $\mathrm{WKB}$ amplitudes

$$
I(q)=\left(\sum_{j=0}^{n-1} \sqrt{\frac{2 \pi}{k_{j}(q)}} \operatorname{sen} \varphi_{j}(q)\right)^{2}
$$

where

$$
k_{j}(q)=\sqrt{\frac{2 M^{2} g}{\hbar^{2}}\left(q+j q_{0}\right)}
$$

and

$$
\varphi_{j}(q)=\int_{-j q_{0}}^{q} k\left(q^{\prime}\right) d q^{\prime}=\int_{-j q_{0}}^{q} \frac{M}{\hbar} \sqrt{2 g\left(q^{\prime}+j q_{0}\right)} d q^{\prime}=\frac{2}{3} \frac{M}{\hbar} \sqrt{2 g}\left(q+j q_{0}\right)^{3 / 2} .
$$

The vertical axis $q$ is oriented downwards and the positions of the leaky condensate portions are $-j q_{0}, j=0, \ldots,(n-$ 1).

A more recent but to a certain extent similar situation occurs in the experiment of experiment of Cataliotti et al.[57], in which a trapped condensate is subjected to a comb of optical barriers before being released. Measured density profiles after an allowed expansion time shows two ejected portions travelling in opposite directions away from a central residual portion. A very schematic "one wavefunction" model for this behavior is provided by the behavior of the density associated with the free evolution of an initial wavefunction given by

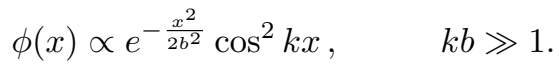

Since $\cos ^{2} k x=(1+\cos 2 k x) / 2$, this wave function is in fact the coherent superposition of three wave packets moving with mean velocities 0 and $\pm 2 \hbar k / M$ respectively. If the $\cos ^{2} k x$ modulation of the gaussian is replaced by a more general periodic function, Fourier analysis would give analogous results involving a richer superposition of differently moving wavepackets.

Still more recently, Greiner et al.[58] observed similarly produced three dimensional arrays of condensate pieces. Here, by varying the barrier height between neighboring sites they have been able to observe interference patterns fol- lowing removal of the trap which changed markedly with the eventual blurring of the interference pattern. This has been interpreted in terms of the confinement of atoms to definite sites in the case of sufficiently high barriers, with loss of definite phase relation between different pieces of the original condensate, in the manner of a bosonic Mott transition.

\subsection{Simple models for split condensates}

The simplest case of a system with many modes is a system with two modes and, appart from its direct relevance to experimental setups like those of refs. [51, 55], it will be useful to analyse this case in detail also in order to see what is really involved and what simplifications can be introduced in the modeling of more complicated situations. Note that the systems considered in section 3.4.1 are in fact two mode systems, but the two modes there refer to two different types of boson (e.g.two different internal states of a given atomic spacies), while here the focus will be on a sigle type of boson evolving in a nearly degenerate pair of spatially orthogonal modes. 


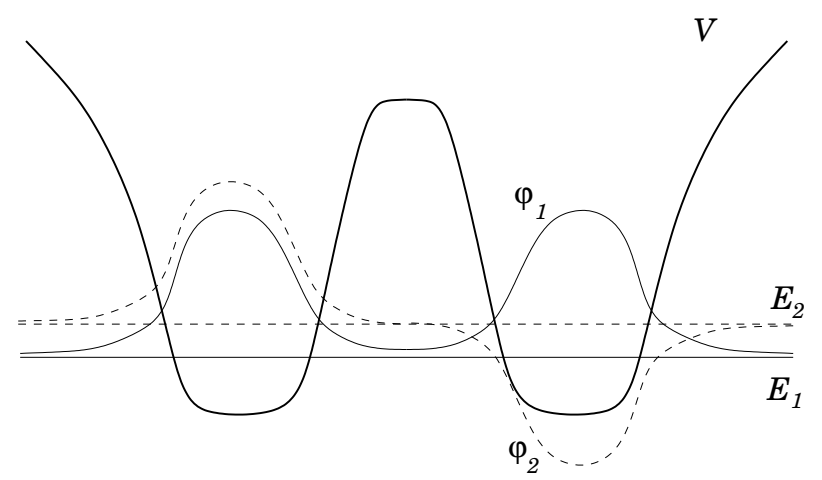

Figure 5.2. Schematic representation of a two-well trap, with the corresponding lowest energy single-particle doublet. The lowest member of the doublet has a nodeless wavefunction $\varphi_{1}$, and the energy splitting $\Delta E \equiv E_{2}-E_{1}$ determines the oscillation period between the two non-stationary, localized states $\left(\varphi_{1} \pm \varphi_{2}\right) / \sqrt{2}$ as $2 \pi \hbar / \Delta E$.

A typical situation of this kind arises in the case of a double-well trap such as the one represented schematically in Fig. 5.2. Laboratory situations are of course threedimensional and can be arranged in a variety of aspect ratios, ranging e.g. from long sausages pinched at the middle by an imposed barrier[51] to also long sausages split lengthwise in half by an interposed wall[55]. Different aspect ratios imply also different energy ratios of excitations in directions transverse to that crossing the barrier, and it will be assumed that these excitations are in any case much higher than the doublet splitting. In this case a possible simplification of the problem consists in restricting the dynamics to a two-mode phase space. This is implemented introducing the creation operators

$$
\begin{gathered}
a_{ \pm}^{\dagger}=\int d^{3} r u\left(r_{\perp}\right) \varphi_{ \pm}(z) \psi^{\dagger}(\vec{r}) \\
\varphi_{ \pm}(z)=\frac{\varphi_{1}(z) \pm \varphi_{2}(z)}{\sqrt{2}}
\end{gathered}
$$

the function $u\left(r_{\perp}\right)$ being the frozen transverse wavefunction and $\varphi_{i}(z), i=1,2$ being the quasi-degenerate longitudinal eigenfunctions for the lowest doublet in the two-well trap. In this way, $\varphi_{ \pm}(z)$ are localized, non-stationary wavefunctions peaking in each of the two sides of the barrier.

The basic hamiltonian to be used to characterize the dynamics of the system is the usual second-quantized effective hamiltonian with the contact two-body effective interaction (2.5) restricted to the two modes. This restriction is easily implemented using for the field operators the substitution

$$
\begin{gathered}
\psi(\vec{r}) \longrightarrow \sum_{ \pm} u\left(r_{\perp}\right) \varphi_{ \pm}(z) a_{ \pm} \\
\psi^{\dagger}(\vec{r}) \longrightarrow \sum_{ \pm} u^{*}\left(r_{\perp}\right) \varphi_{ \pm}^{*}(z) a_{ \pm}^{\dagger}
\end{gathered}
$$

which, after some trimmings to be discussed shortly, gives

$$
\begin{aligned}
H_{\mathrm{eff}} \stackrel{\sim}{\longrightarrow} H_{B H} \equiv & E_{+} a_{+}^{\dagger} a_{+}+E_{-} a_{-}^{\dagger} a_{-}+\alpha\left(a_{+}^{\dagger} a_{-}+a_{-}^{\dagger} a_{+}\right)+ \\
& +\frac{\Lambda_{+}}{2} a_{+}^{\dagger} a_{+}^{\dagger} a_{+} a_{+}+\frac{\Lambda_{-}}{2} a_{-}^{\dagger} a_{-}^{\dagger} a_{-} a_{-} .
\end{aligned}
$$

Here the constants $E_{ \pm}$stand for the diagonal matrix elements

$$
E_{ \pm}=\left\langle u \varphi_{ \pm}\left|\frac{p^{2}}{2 M}+V\right| u \varphi_{ \pm}\right\rangle
$$

Note that if the delocalized functions $u \varphi_{i}, i=1,2$ are taken as eigenfunctions of $p^{2} / 2 M+V$ with eigenvalues $E_{i}$, then $E_{+}=E_{-}=\left(E_{1}+E_{2}\right) / 2$. The parameter $\alpha$ stands for the off-diagonal matrix elements

$$
\alpha=\left\langle u \varphi_{ \pm}\left|\frac{p^{2}}{2 M}+V\right| u \varphi_{\mp}\right\rangle,
$$

taken to be equal, the wavefunctions being both real. Again, for eigenfunctions of $p^{2} / 2 M+V$ one finds that $\alpha=\left(E_{1}-\right.$ $\left.E_{2}\right) / 2<0$. This reveals, in particular, that this term is related to the periodic tunneling of partiches across the barrier.

The two-body part in fact gives rise to sixteen terms involving space integrals of the various distinct products of four mode wavefunctions $u \varphi_{ \pm}$. Due to the localized character of these wavefunctions, however, integrals over products of four wavefunctions that are not all equal are much smaller than the two which have been retained. The parameters $\Lambda_{ \pm}$ are therefore essentially equal and given by

$$
\Lambda_{ \pm}=\lambda \int d^{3} r u^{4}\left(r_{\perp}\right) \varphi_{ \pm}^{4}(z) .
$$

The trimmed hamiltonian (5.2) is nothing but a bosonic version of a two-site Hubbard model (in short, the two-site Bose-Hubbard model), in which the "hopping" term relates to tunneling between different wells and there is an "in site" two-body interaction, in addition to possibly different onebody site energies. The above "derivation" can moreover be extended to more sites and/or dimensions, by picking Wanier functions[59] as the appropriate generalization of the two-well localized wavefunctions. The standard form of the trimmed model hamiltonian is 


$$
H_{B H}=\sum_{i} E_{i} a_{i}^{\dagger} a_{i}+\alpha \sum_{<i, j>}\left(a_{i}^{\dagger} a_{j}+a_{j}^{\dagger} a_{i}\right)+\frac{\Lambda}{2} \sum_{i} a_{i}^{\dagger} a_{i}^{\dagger} a_{i} a_{i},
$$

where $\langle i, j\rangle$ in the second sum denotes that the sites $i$ and $j$ are neighbors.

This argument shows therefore that, in presence of a spatially periodic trap potential which gives rise to a large number of quasi-degenerate independent modes one can, by restricting the phase space to just the quasi-degenerate modes, using a representation in terms of localized wavefunctions (typically a "single" Wanier function) one is led, with only some mild trimmings, from the Gross-Pitaevski problem to the Bose-Hubbard model. The restriction to the quasi-degenerate modes is the most severe limitation of the Bose-Hubbard model. This restriction in fact constrains the rendering of the dependence of effective mean-field properties on degrees of freedom kept active in the model, such as mean occupations of the different sites. While in the GrossPitaevski treatment this is taken into account by the nonlinear term, in the Bose-Hubard model it is limited by freezing once and for all a set of quasi-degenerate orbitals, such as the wavefunctions $u \varphi_{1}$ and $u \varphi_{2}$ in the case of the two-well trap of Fig. 5.2.

\subsubsection{Results for a two-well system}

In this case one considers the hamiltonian (5.2) with $E_{+}=$ $E_{-}=0$ and $\Lambda_{+}=\Lambda_{-}=\Lambda$, i.e.

$$
H_{B H}=\alpha\left(a_{+}^{\dagger} a_{-}+a_{-}^{\dagger} a_{+}\right)+\frac{\Lambda}{2}\left(a_{+}^{\dagger} a_{+}^{\dagger} a_{+} a_{+}+a_{-}^{\dagger} a_{-}^{\dagger} a_{-} a_{-}\right) .
$$

and realizes imediately to have been left with a particular case of the hamiltonian that was considered in section 3.4.1. In particular, since the confining trap is taken into account in terms of the choice made for the relevant orbitals $u \varphi_{i}$, $i=1,2$, there is no explicit reference to the position degree of freedom besides that which is related to the indices \pm and the localized character of the corresponding orbitals. Furthermore, there are no two body interactions between bosons in different sites, and the total number of bosons

$$
\hat{N}=a_{+}^{\dagger} a_{+}+a_{-}^{\dagger} a_{-}
$$

is clearly a constant of motion.

A convenient way of dealing with the two-mode hamiltonian (5.5) within a sector of the second-quantized phase space having a definite number of particles $N$ is to define the operators

$$
J_{ \pm}=J_{1} \pm i J_{2} \equiv a_{ \pm}^{\dagger} a_{\mp},
$$

$$
\begin{gathered}
J_{3} \equiv \frac{a_{+}^{\dagger} a_{+}-a_{-}^{\dagger} a_{-}}{2}, \\
J \equiv \frac{a_{+}^{\dagger} a_{+}+a_{-}^{\dagger} a_{-}}{2}=\frac{\hat{N}}{2},
\end{gathered}
$$

which constitute Schwinger's well known realization of the angular momentum algebra it terms of two bosonic modes[60]. They are therefore frequently refered to as "quasi-spin" operators in this context[31]. The role played by the operator $J$ is revealed by the relation

$$
J_{1}^{2}+J_{2}^{2}+J_{3}^{2}=J(J+1)=\frac{\hat{N}}{2}\left(\frac{\hat{N}}{2}+1\right)
$$

so that the value of the quasi-spin is half the number of bosons in the considered sector. The two-site hamiltonian (5.5) can be expressed in terms of the quasi-spin operators as

$$
\begin{aligned}
H_{B H} & =2 \alpha J_{1}+\frac{\Lambda}{2}\left[\left(J+J_{3}\right)\left(J+J_{3}-1\right)+\left(J-J_{3}\right)\left(J-J_{3}-1\right)\right] \\
& =2 \alpha J_{1}+\Lambda J(J-1)+\Lambda J_{3}^{2} .
\end{aligned}
$$


The second term is a constant of motion, and the two remaining terms are non-commuting cartesian components of the quasi-spin. This reveals at once that one can diagonalize the hopping term proportional to $\alpha$ by choosing a representation in terms of the simultaneous eigenvectors of the square of the total spin and of the component $J_{1}$, and in this case the on-site two-body effective interaction term will have off-diagonal matrix elements. Or, alternatively, one can diagonalize the on-site two-body effective interaction term, by choosing instead the representation in which $J(J-1)$ and $J_{3}$ are diagonal, and in this case the hopping term will have off diagonal matrix elements. The ground state for $\alpha=0$, which implies a completely impermeable barrier, is the state $\left|J=N / 2, J_{3}=0\right\rangle$ which corresponds to an equal number of bosons in each well (assuming $N$ to be even, and a repulsive effective two-body interaction). Conversely, the ground state in the case $\Lambda=0$, and assuming $\alpha<0$ (cf. the discussion following eq. (5.3)), is the state $\left|J=N / 2, J_{1}=N / 2\right\rangle$.
To see what this state is, recall the relations between the localized wavefunctions $u \varphi_{ \pm}$to the quasi-degenerate, delocalized wavefunctions $u \varphi_{1,2}$ which imply the relations

$$
a_{ \pm}=\frac{a_{1} \pm a_{2}}{\sqrt{2}}
$$

where the anihilation operators $a_{1,2}$ are associated to the quasi-degenerate single particle state rather than with the localized states. Straightforward algebra then gives immediately

$$
2 J_{1}=a_{+}^{\dagger} a_{-}+a_{-}^{\dagger} a_{+}=a_{1}^{\dagger} a_{1}-a_{2}^{\dagger} a_{2},
$$

so that the eigenstate $\left|J=N / 2, J_{1}=N / 2\right\rangle$ is the state in which all $N$ particles are in the lowest one of the quasidegenerate single particle doublet, and therefore fully delocalized. It can be expressed in terms of the common normalized eigenvectors of $J(J+1)$ and $J_{3}$ after a simple calculation:

$$
\begin{aligned}
\left|J=N / 2, J_{1}=N / 2\right\rangle & =\frac{1}{\sqrt{N !}}\left(a_{1}^{\dagger}\right)^{N}|0\rangle=\frac{1}{\sqrt{N !}}\left(\frac{a_{+}^{\dagger}+a_{-}^{\dagger}}{\sqrt{2}}\right)^{N}|0\rangle= \\
& =\frac{1}{2^{N / 2} \sqrt{N !}} \sum_{n=0}^{N}\left(\begin{array}{c}
N \\
n
\end{array}\right)\left(a_{+}^{\dagger}\right)^{N-n}\left(a_{-}^{\dagger}\right)^{n}|0\rangle= \\
& =\frac{1}{2^{N / 2}} \sum_{n=0}^{N} \sqrt{\left(\begin{array}{c}
N \\
n
\end{array}\right)}\left|J=N / 2, J_{3}=(N-2 n) / 2\right\rangle
\end{aligned}
$$

It will be convenient to refer to the alternate representations in which the hopping term and the on-site two body effective interaction are diagonal simply as the $J_{1}$ representation and the $J_{3}$ representation respectively.

The preceding discussion provides enough elements for a qualitative understanding of the dynamics implied by the model hamiltonian (5.5). One sees that the hopping term favors delocalization of the particles so as to promote the relevance of the lowest member of the quasi-degenerate doublet; and that, on the contrary, the on-site two body effective interaction favors the most symmetric state of the $J_{3}$ representation, having $J_{3}=0$, which means half of the particles localized in each of the two wells. In general the spectrum of $H_{B H}$ will consist of $N+1$ states in the $N$ boson sector. The eigenvalues and eigenvectors can be obtained by diagonalizing the hamiltonian matrix in any of the two representations.

\subsubsection{Semi-classical domain}

A semi-classical domain exists for $N \gg 1$, and in this case a formulation of the dynamics in classical terms may be useful. To this effect, consider, instead of the three operators $J_{k}, k=1,2,3$, which have eigenvalues in the interval $-J$ to $+J$, their scaled couterparts

$$
j_{k} \equiv \frac{J_{k}}{J}, \quad J=\frac{N}{2}
$$

which have eigenvalues in the range -1 to +1 . The spectrum of the scaled operators therefore becomes very dense in the semiclassical regime and may be treated as a continuous variable. At the same time, from the angular momentum commutation relations satisfied by the $J_{k}$, namely

$$
\left[J_{j}, J_{k}\right]=i \epsilon_{j k l} J_{l},
$$

where $\epsilon_{j k l}$ is the completely antisymmetric symbol, it follows that

$$
\left[j_{j}, j_{k}\right]=\frac{2 i}{N} \epsilon_{j k l} j_{l}
$$

so that the scaled operators approximately commute in the semiclassical domain $N \gg 1$. Furthermore, in this domain the object

$$
\left\{j_{j}, j_{k}\right\} \equiv \frac{N}{2 i}\left[j_{j}, j_{k}\right]=\epsilon_{j k l} j_{l}
$$

plays the role of the Poisson brackets of the dynamical variables represented by the scaled operators. The quasi-spin 
hamiltonian per particle can now be written in terms of the scaled operators as

$$
\frac{1}{2 J} H_{B H} \equiv h_{B H}=\alpha j_{1}+\frac{\Lambda N}{4}\left(1+\frac{1}{N}\right)+\frac{\Lambda N}{4} j_{3}^{2} .
$$

Recall that the parameter $\Lambda$ is related to the "basic" strength parameter $\lambda=4 \pi \hbar^{2} a / M$ of the effective two body interaction by an integral over the fourth power of the mode wavefunctions, which is if the order of magnitude of the inverse volume of the system. Therefore $\Lambda N$ is of the order of the constant $\lambda$ times the mean density of the system.

To the extent that the $j_{i}$ can be treated as classical variables, this hamiltonian can be used to obtain their equations of motion by using the Poisson brackets (5.8). In this way one obtains

$$
\frac{d j_{i}}{d t}=\left\{j_{i}, h_{B H}\right\}, \quad i=1,2,3
$$

from which it it easy to check that $\sum_{i=1}^{3} j_{i}^{2}$ is a (classical) constant of motion. The variables $j_{i}$ are in fact the cartesian components of a unit, classical quasi-spin vector which rotates in time as dictated by the equations of motion. The variable which is canonically conjugated to $j_{3} \equiv \cos \theta$ is the azimuthal angle $\varphi$ of this rotating vector, $\theta$ being the usual colatitude. The "classical" hamiltonian (cf. ref. [61]) can thus be written in terms of canonical variables $j_{3}, \varphi$ as

$$
h_{B H}^{(\mathrm{cl})}=\frac{\Lambda N}{4}\left(1+\frac{1}{N}+j_{3}^{2}\right)+\alpha \sqrt{1-j_{3}^{2}} \cos \varphi .
$$

Here the relation $\sin \theta=\sqrt{1-j_{3}^{2}}$ has been used. Note that, since $0 \leq \theta \leq \pi$, the suare root must be taken with positive sign.

The canonical equations of motion are

$$
\begin{aligned}
\frac{d \varphi}{d t} & =\frac{\Lambda N}{2} j_{3}-\alpha \frac{j_{3}}{\sqrt{1-j_{3}^{2}}} \cos \varphi \\
\frac{d j_{3}}{d t} & =\alpha \sqrt{1-j_{3}^{2}} \sin \varphi
\end{aligned}
$$

and the classical stationary states are at the values of $\varphi$ and $j_{3}$ for which the time derivatives vanish. This implies $\sin \varphi=0$, so that $\varphi=0$ or $\varphi=\pi$, the coresponding values of $j_{3}$ being respectively the solutions of

$$
j_{3}\left(\frac{\Lambda N}{2}-\frac{\alpha \cos \varphi}{\sqrt{1-j_{3}^{2}}}\right)=0, \quad \cos \varphi= \pm 1 .
$$

Thus solutions $j_{3}=0$ with $\alpha=0$ or $\pi$ always exist, other solutions being given by $\sqrt{1-j_{3}^{2}}=2 \alpha \cos \varphi / \Lambda N$. For $\alpha<0$ only the possibility $\varphi=\pi$ remains for these other solutions, which furthermore will exist as real numbers only if $4 \alpha^{2}<\Lambda^{2} N^{2}$. The minimum energy static solution for $\alpha<0$ is at $j_{3}=0, \varphi=0$. Other solutions correspond in this case to maxima or saddle points of the hamiltonian surface in the $j_{3} \times \varphi$ phase space. An example of the surface representing the classical hamiltonian (per particle) (5.9) can be seen in Fig. 5.5(a) below.

It is worth noting that these semi-classical results can also be obtained by using as ansatz for the hamiltonian (5.5) a product of coherent states for the two modes

$$
\left|z_{+} z_{-}\right\rangle \equiv e^{z_{+} a_{+}^{\dagger}-z_{+}^{*} a_{+}} e^{z_{-} a_{-}^{\dagger}-z_{-}^{*} a_{-}}|0\rangle
$$

and introducing explicitly the constraint $\left|z_{+}\right|^{2}+\left|z_{-}\right|^{2}=N$ together with the definition $j_{3} \equiv\left(\left|z_{+}\right|^{2}-\left|z_{-}\right|^{2}\right) / N$. The expectation value of the hamiltonian (5.5) turns out to be just (5.9), the angle variable $\varphi$ being the relative phase between the two coherent condensates, $\varphi=\operatorname{Arg}\left(z_{+} z_{-}^{*}\right)$. This then provides for an interpretation of the angle variable of the quasi-spin treatment. The fact thet this angle variable is canonically conjugate to $j_{3}$, which measures the population inbalance of the two localized states, indicates that, in a quantum regime, the population inbalance and the relative phase of the condensates are quantities which maintain complementarity relations to each other.

\subsubsection{Quantum domain}

The quantum mechanical ground state of the two-site BoseHubbard hamiltonian in either of the forms (5.5) or (5.6) cannot be associated with a sharp value of $J_{3}$ (i.e., of the population inbalance) due to the presence in the hamiltonian of the hopping term, proportional to $J_{1}$ and therefore non commuting with $J_{3}$. Since the total number of particles is a constant of motion, this means that there will be correlated quantum fluctuations of the two number operators, $a_{+}^{\dagger} a_{+}$and $a_{-}^{\dagger} a_{-}$.

The quasi-spin representation, including the fact that the operator $J(J+1)$ is a constant of motion, makes the problem "soluble", in the sense that eigenvalues and eigenvectors can be obtained from the diagonalization of finite matrices. In such a numerical procedure, the eigenvectors in the $J_{3}$ representation

$$
H_{B H}\left|E_{n}\right\rangle=E_{n}\left|E_{n}\right\rangle, \quad\left|E_{n}\right\rangle=\sum_{m=-J}^{J} c_{m}^{(n)}|J, m\rangle,
$$

give directly the corresponding distributions of the occupation ratios for the two localized states in the form of the arrays of $2 J+1=N+1$ numbers $\left\{\left|c_{m}^{(n)}\right|^{2}\right\}$. The corresponding distribution in the relative phase between the two condensates, which is conjugate to the distribution of occupation inbalance, can be obtained by taking a discrete Fourier transform of the energy eigenvectors in the $J_{3}$ representation

$$
\tilde{c}_{\nu}^{(n)}=\frac{1}{\sqrt{2 J+1}} \sum_{m=-J}^{J} e^{\frac{2 \pi i \nu m}{2 J+1}} c_{m}^{(n)}, \quad-J \leq \nu \leq J
$$

It is then given by the array $\left\{\left|\tilde{c}_{\nu}^{(n)}\right|^{2}\right\}$, which is complementary to the array which describes the distribution of occupation ratios. Examples of such distributions are shown in 

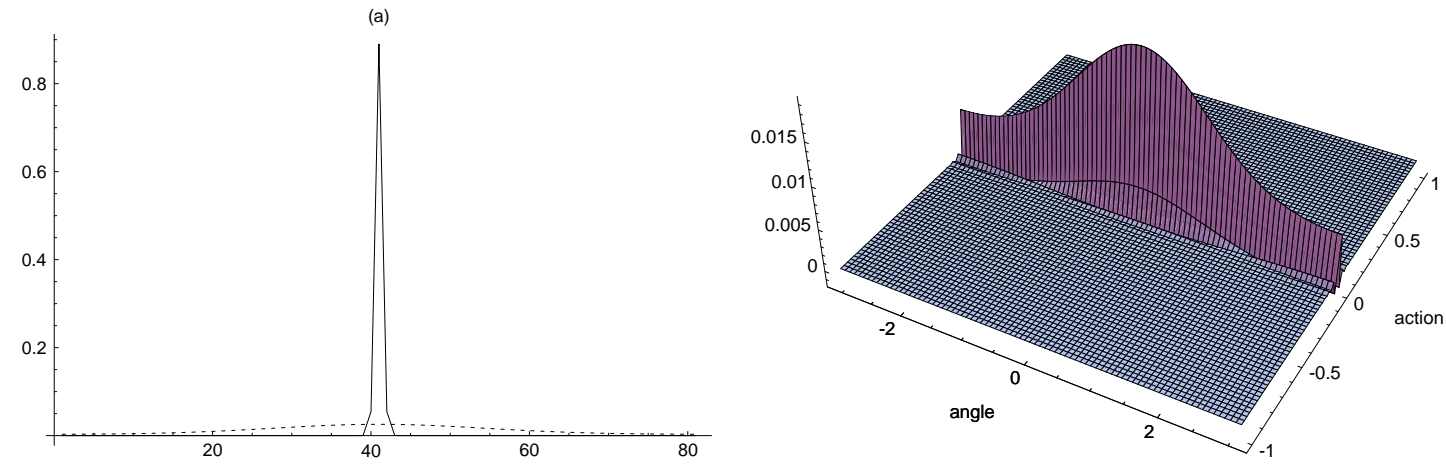

(b)
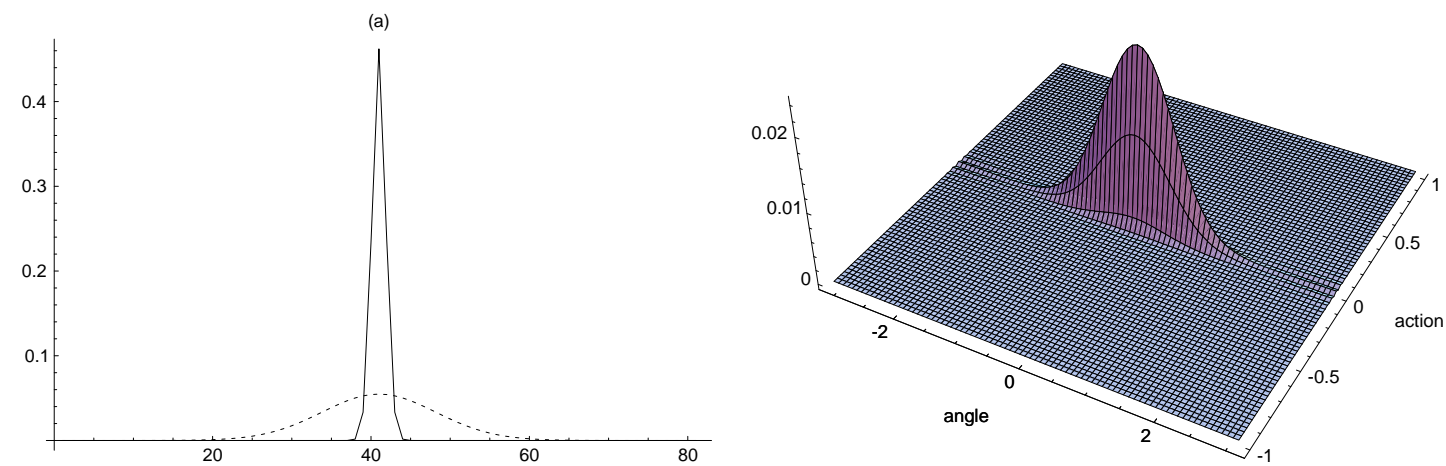

Figure 5.3. Top: (a)Distributions of population inbalance (full line) and relative phase (dashed line) for the ground state of the two site Bose-Hubbard model with $N=80, \alpha=-1.0 \times 10^{-4} \mathrm{~Hz}$ and $\Lambda=0.0146 \mathrm{~Hz}$ ). In the case of the population inbalance, the labels on the horizontal axis are to be read as $2(m+J),-J \leq m \leq J=40$. In the case of the relative phase distribution they are to be read as $40(1+\varphi / \pi)$, which corresponds to $\varphi=-\pi$ at the origin and $\varphi=\pi$ at full scale; (b) Discrete Wigner function for the same case. The axis labeled "action" refers to $j_{3}$, that labeled "angle"refers to $\varphi$. Bottom: Same as the top figures, with the barrier somewhat lowered ( $\Lambda$ unchanged, $\alpha=-1.9 \times 10^{-3} \mathrm{~Hz}$ ). One sees clearly the better definition of the relative phase and some loss in the definition of the population inbalance between the two wells.

Fig. 5.3(a) for two different heights of the interwell potential barrier. Note that these are discrete distributions, whose entries are labeled respectively by the eigenvalue $m$ of $J_{3}$ (or, equivalently, by $2 m / N$ ) and by the discrete set of angles $2 \pi m /(2 J+1)=2 \pi m /(N+1)$, which appear in the discrete Fourier transform.

Alternatively, one can visualize both distributions simultaneously by calculating a discrete Wigner distribution di- rectly from the amplitudes which appear as expansion coefficients of the considered energy eigenstate $\left|E_{n}\right\rangle$ in the $J_{3}$ representation as in eq. (5.10). This procedure has been used in ref ([31]) and is discussed in detail in ref. [62]. It is implemented basically in the following steps. Given the array of aplitudes $\left\{c_{m}^{(n)}\right\},-J \leq m \leq J$, and assuming that $J$ is an integer, which implies that $N$ is even, one first constructs the matrix

$$
r(k, l)=\frac{1}{\sqrt{2 J+1}} \sum_{m=-J}^{J} c_{m}^{(n)} c_{\{m+l\}}^{(n) *} \exp \left[-\frac{2 \pi i}{2 J+1} k\left(m+\frac{l}{2}\right)\right]
$$

where the range of the integers $k$ and $l$ is $-J \leq k, l \leq J$ and the index $\{m+l\}$ denotes the value of $m+l$ cyclically confined to the range $-J, J$ of the basis labels. Explicitly, one has

$$
\{m+l\}=m+l-(2 J+1) \text { Floor }\left(\frac{m+l+J}{2 J+1}\right)
$$


where Floor $(x)$ denotes the largest integer (negative for $x<0$ ) less than or equal to $x$. The desired discrete Wigner phasespace representative $c_{w}^{(n)}(p, q)$ of the state $\left|E_{n}\right\rangle$ is then obtained as the double (discrete) Fourier transform

$$
(2 J+1) a_{w}(p, q)=\frac{1}{\sqrt{2 J+1}} \sum_{k, l} \exp \left[\frac{2 \pi i}{2 J+1}(p k+q l)\right] r(k, l) .
$$

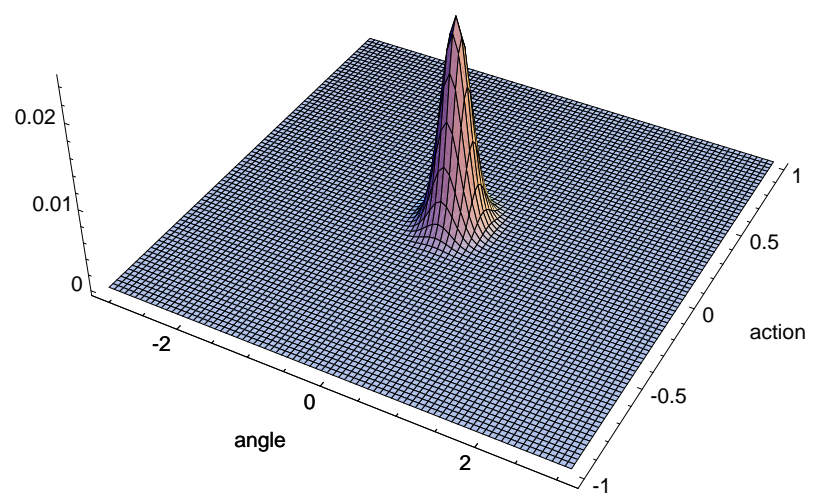

Figure 5.4. Discrete Wigner function for the ground state of the two-site Bose-Hubbard hamiltonian for $N=80, \Lambda=0.0146$ and $\alpha=-0.1$. The classical lowest energy state is represented in this phase-space diagram by the single point $\varphi=0, j_{3}=0$. Increasing the number of particles decreases the relative variances of the population inbalance and relative phase distributions.

In this expression the range of the integers $p$ and $q$ is also bounded as $-J \leq p, q \leq J$, and the properly scaled variables corresponding to $j_{3}$ and $\varphi$ are $q / J$ and $2 \pi p /(2 J+1)$ respectively. The Weyl transform of the hamiltonian can be obtained in exactly the same way, replacing the amplitude products $c_{m}^{(n)} c_{\{m+l\}}^{(n) *}$ by the matrix elements $\left\langle J, m\left|h_{B H}\right|\right.$ $J,\{m+l\}\rangle$ multiplied by the number of states $2 J+1$, when evaluating $r(k, l)$.

Figure 5.3(b) shows the discrete Wigner functions for the ground states whose population inbalance and relative phase distributions are shown in the parts (a). The latter can in fact be obtaining by summing the Wigner function over the complementary variable. The case of a still stronger hopping term, $\alpha=.1$, also for $N=80$, is shown in Fig. 5.4. The Weyl transform of the corresponding quantum hamiltonian is shown in Fig. 5.4(b), while part (a) of the same figure shows its classical version.

There is a somewhat subtle property of the hamiltonian which gives rise to an important property of its eigenstates, concerning the experimentally important class of one-body observables. This property is best expressed in terms of the number operators for the $J_{1}$ representation, namely those associated with the delocalized members $u \varphi_{i}, i=1,2$ of the active doublet, $a_{i}^{\dagger} a_{i}, i=1,2$. It consists in the fact that, even though these number operators are not themselves constants of motion, the hamiltonian does not mix eigenstates of either of them whose eigenvalues differ by an odd number of particles; or, in different words, the hamiltonian admits the modular constants of motion

$$
\begin{gathered}
P_{i} \equiv(-1)^{a_{i}^{\dagger} a_{i}}, \quad i=1,2, \quad \text { i. e. } \\
{\left[P_{i}, H_{B H}\right]=0, \quad i=1,2 .}
\end{gathered}
$$

This property can be easily verified by re-expressing the hamiltonian (5.5) in terms of the operators $a_{i}, a_{i}^{\dagger}$ and noting that each term either maintains the occupancy of each state fixed or changes it by two particles. Due to the conservation of the total number of particles the number operators $a_{i}^{\dagger} a_{i}$, $i=1,2$ are themselves correlated, so that it is sufficient to consider just one of them, say $a_{1}^{\dagger} a_{1}$.
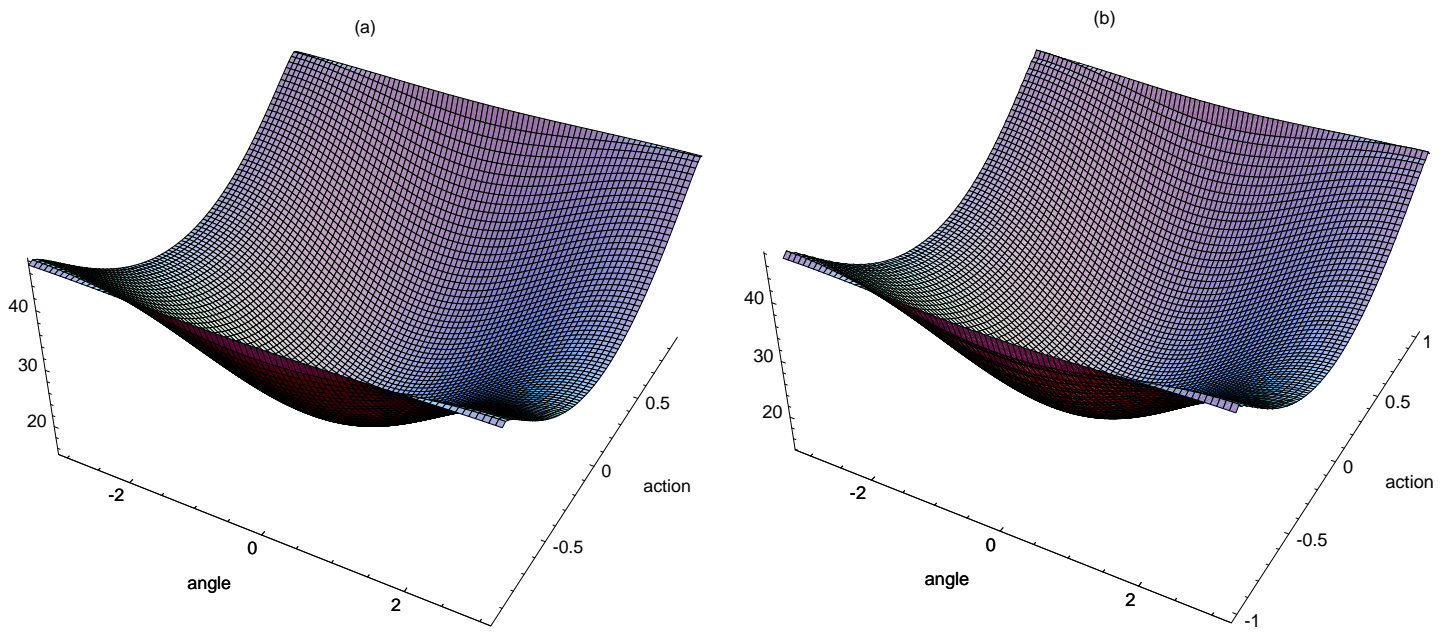

Figure 5.5. (a) Energy surface representing the classical Bose-Hubbard hamiltonian for two sites, eq. (5.9) multiplied by the number of particles $(N=80)$, for $\Lambda=0.0146$ and $\alpha=-0.1$; (b) Discrete Weyl transform of the quantum hamiltonian for $N=80$ and for the same values of $\Lambda$ and $\alpha$. 
As a consequence of this property, the eigenvectors of (5.5) can be chosen to be simultaneous eigenvectors of $P_{1}$ (they will automatically fulfill this property except for reasons of degeneracy) and as such divided in two classes according to the eigenvalues of the modular constants of motion. In particular, a non-degenerate ground state $\left|\Phi_{0}\right\rangle$ will be an eigenstate of $P_{1}$. This has important consequences for its one-particle density matrix

$$
\rho\left(\vec{r}, \vec{r}^{\prime}\right) \equiv\left\langle\Phi_{0}\left|\psi^{\dagger}\left(\vec{r}^{\prime}\right) \psi(\vec{r})\right| \Phi_{0}\right\rangle,
$$

which in the case in hand can be expressed as a two by two matrix in the $J_{1}$ representation

$$
\rho=\left(\begin{array}{cc}
\left\langle\Phi_{0}\left|a_{1}^{\dagger} a_{1}\right| \Phi_{0}\right\rangle & \left\langle\Phi_{0}\left|a_{2}^{\dagger} a_{1}\right| \Phi_{0}\right\rangle \\
\left\langle\Phi_{0}\left|a_{1}^{\dagger} a_{2}\right| \Phi_{0}\right\rangle & \left\langle\Phi_{0}\left|a_{2}^{\dagger} a_{2}\right| \Phi_{0}\right\rangle
\end{array}\right)
$$

In fact, the operators appearing in the off-diagonal matrix elements change the eigenvalue of $P_{1}$, and therefore their expectation value vanishes when taken with respect to an eigenstate of this operator.

As a consequence of the modular constant of motion $P_{1}$ we find therefore that the one body density matrix is diagonal in the $J_{1}$ representation. This means that the delocalized states $u \varphi_{i}, i=1,2$ are the natural orbitals which carry the coherence properties which are relevant for one-body observables. Correlations introduced into the ground state by the non comitativity of the hopping term and the on-site twobody effective interaction will affect just the relative weights of the two quasi-degenerate modes. This immediately suggests a considerable stability of any one body interference patterns associated with the nature of the doublet wavefunctions, the effect of many-body correlations being manifest rather in their visibility. These patterns are in fact entirely determined by the one body density matrix, wuich in the $J_{1}$ representation takes the form

$$
\rho=A \hat{\mathbf{1}}+B\left|u \varphi_{1}\right\rangle\left\langle u \varphi_{1}\right|
$$

where $\hat{\mathbf{1}}$ is the unit $2 \times 2$ matrix. Since $\operatorname{Tr} \rho=N$, one has $2 A+B=N$ and $J_{1}=B / 2$, or $j_{1}=B / N$, and the parameter relevant for the visibility is $J_{1}$ (or $j_{1}$, or $B$ ). For the two cases shown in Fig. 5.3, in which $N=80$ and the dominant term in the hamiltonian is the on site two body effective interaction, with $\Lambda=0.0146 \mathrm{~Hz}$, one has $B=36.3$ (for $\alpha=1.0 \times 10^{-4} \mathrm{~Hz}$ ), which corresponds to $j_{1}=.454$, and $B=68.9$ (for $\alpha=1.0 \times 10^{-3} \mathrm{~Hz}$ ), which corresponds to $j_{1}=.860$.

\subsection{Many site one dimensional arrays, peri- odic boundary conditions}

A more general class of systems to which many of the above results still apply is that of one-dimensional arrays of condensate fractions with periodic boundary conditions, which has been studied recently, form a mean-field point of view, by Paraoanu[63]. The idea is to arrange $\nu_{c}$ condensate fractions in a "circular array", so that the the the first one is also next to the $\nu_{c}$-th one. The Bose-Hubbard hamiltonian for this system has the form (5.4) with degenerate sites $\left(E_{i}=0\right.$ for $i=1, \ldots, \nu_{c}$ ), i.e.

$$
H_{B H}^{\left(\nu_{c}\right)}=\alpha \sum_{<i, j>}\left(a_{i}^{\dagger} a_{j}+a_{j}^{\dagger} a_{i}\right)+\frac{\Lambda}{2} \sum_{i=1}^{\nu_{c}} a_{i}^{\dagger} a_{i}^{\dagger} a_{i} a_{i}
$$

where the sum over nearest neighbors includes the term $a_{1}^{\dagger} a_{\nu_{c}}+a_{\nu_{c}}^{\dagger} a_{1}$. The operators $a_{i}, a_{i}^{\dagger}, i=1, \ldots, \nu_{c}$ refer to the single particle states of a localized (Wanier) base. One car however introdice a complementary base by defining the alternate creation and anihilation operators

$$
A_{n}^{\dagger} \equiv \sum_{k=1}^{\nu_{c}} e^{-\frac{2 \pi i n k}{\nu_{c}}} a_{k}^{\dagger}, \quad n=0, \ldots, \nu_{c}-1
$$

in terms of which the hamiltonian becomes

$$
H_{B H}^{\left(\nu_{c}\right)}=2 \alpha \sum_{n=0}^{\nu_{c}-1} \cos \frac{2 \pi n}{\nu_{c}} A_{n}^{\dagger} A_{n}+\frac{\Lambda}{\nu_{c}} \sum_{n, p, q=0}^{\nu_{c}-1} A_{q+n}^{\dagger} A_{p-n}^{\dagger} A_{p} A_{q}
$$

i.e., in the complementary base the hopping term is diagonal. The indices $n, p$ and $q$ in the two body term run from 0 to $\nu_{c}-1$, and the indices $q+n, p-n$ are to be understood as modulo $\nu_{c}$ (e.g. $\nu_{c}+2 \equiv 2,-\nu_{c}+1 \equiv 1, \pm \nu_{c} \equiv 0$ ). The structure of this term therefore reveal the conservation of the "modular momentum" associated to the index of the creation operators of the complementary base.

Thus the complementary base plays in this case the same role as the $J_{1}$ representation in the two-site case. Not only it diagonalizes the pure hopping $(\Lambda=0)$ hamiltonian, but also diagonalizes the one-body density matrix associated with the simultaneous eigenstates of $H_{B H}$ and of this "modular momentum". This constant of motion allows now for the classification of the energy eigenstates of the model in $\nu_{c}$ classes according to the value of the total modular momentum. Thus, for an eigenstate $\left|\Phi_{0}\right\rangle$,

$$
\rho_{p q} \equiv\left\langle\Phi_{0}\left|A_{q}^{\dagger} A_{p}\right| \Phi_{0}\right\rangle=n_{p} \delta_{p q} .
$$

In fact, when $q \neq p$ the operator defining the one body density matrix element changes the value of the total modular 
momentum of $\left|\Phi_{0}\right\rangle$, so that the coresponding matrix element vanishes. One has therefore again a situation showing marked propensity for the preservation of one-body interference patterns, even if with varying degrees of visibility, as the competition of hopping and on site two body interactions reduces the coherence of the one body density matrix.

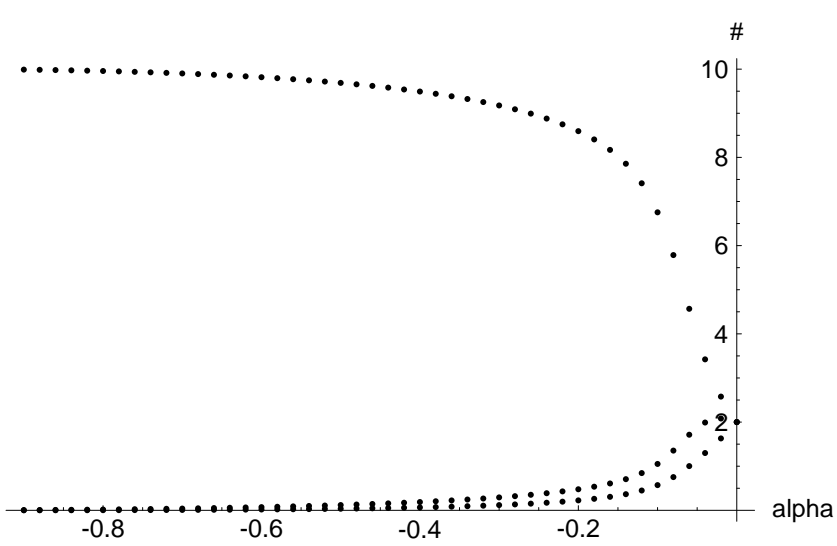

Figure 5.6. Dependence of occupation numbers \#(diagonal matrix elements of the one body density matrix in the complementary representation) with hopping parameter $\alpha$. The two body interaction parameter is $\Lambda=1+\alpha$. Occupation numbers are calculated with a mesh of 0.1 in $\alpha$. In this case $N=10$ and $\nu_{c}=5$. The two lowest occupations are doubly degenerate each and correspond to the $n \neq 0$ states. The upper points corespond to the $n=0$ state.

The hamiltonians (5.12) and (5.13) are also "soluble" in the same sense as the two site Bose-Hubbard hamiltonian, i.e., eigenvalues and eigenvectors can be obtained by numerical diagonalization of finite (if possibly very large) matrices. These methods again indicate considerable permanence of large occupations of the $n=0$ state of the complementary base in the ground state one body density matrix up to fairly strongly dominating on site two body interaction effects. An example of this is shown in Fig. 5.6, where results for a loop containing five condensate fragments with a total of ten particles are shown. The occupations of the five different states of the complementary base are such that those corresponding to $n$ and $\nu_{c}-n$ are equal. This then gives in general three different occupation numbers, two of which appearing twice. The non doubled occupation os that for $n=0$, which dominates over the range $|\alpha| / \Lambda>\sim 0.1$.

\section{References}

[1] A. F. R. de Toledo Piza, Mecânica Quântica, EDUSP, São Paulo, 2003.

[2] Tino Weber, Jens Herbig, Michael Mark, Hanns-Christoph Ngerl

and Rudolf Grimm, Sciencexpress/ www.sciencexpress.org / 5 December 2002 / Page 1/ 10.1126/science.1079699

[3] S. Inouye, M. R. Andrews, J. Stenger, H.-J. Miesner, D. M. Stamper-Kurn, and W. Ketterle, Nature 392, 151 (1998). See also K. Burnett ibid. page 125.
[4] J. L. Roberts, N. R. Clausen, J. P. Burke, C. H. Greene, E. A. Cornell, and C. E. Wieman, Phys. Rev. Letters 81, 5109 (1998).

[5] S. L. Cornish, N. R. Claussen, J. L. Roberts, E. A. Cornell, and C. E. Wieman, Phys. Rev. Letters 85, 1795 (2000).

[6] R. P. Feynman, Statistical Mechanics - A set of lectures, W. A. Benjamin, Inc., Reading, Massachusetts, 1972.

[7] W. Ketterle and N. J. van Druten, Phys. Rev. A54, 656 (1996).

[8] C. Cohen-Tannoudji, La condensation de Bose-Einstein: Introduction, in Séminaire Poincaré Mars 2003, Vol. 1, 1 (2003). I am indebted to Walter Wreszinski for making this reference available to me.

[9] E. Abramowitz and I. A. Stegun, Handbook of mathematical functions, Dover, N.Y., 1965, Ch. 23.

[10] Ph. W. Courteille, V. S. Bagnato, and V. L. Yukalov, Laser Physics 11659 (2001).

[11] See A. L. Fetter and J. D. Walecka, Quantum Theory of Many-Particle Systems, MacGraw-Hill Book Co., 1971, section 41, and references therein, especially the "anschaulich" treatment of L. C. Gomes, J. D. Walecka, and V. Weisskopf, Ann. Phys. (N.Y.) 3, 241 (1958).

[12] K. A. Brueckner and K. Sawada, Phys. Rev. 106, 1117 and 1128 (1957); K. Sawada, Phys. Rev. 116, 1344 (1959).

[13] C. A. Sacket, C. C. Bradley, M. Welling, and R. G. Hulet, Appl. Phys. B65, 433 (1997); C. A. Sacket, J. M. Gerton, M. Welling, and R. G. Hulet, Phys. Rev. Lett. 82, 876 (1999); J. M. Gerton, D. Strekalov, I. Prodan, and R. G. Hulet, Nature 408, 692 (2000).

[14] A. Gammal, L. Tomio, and T. Frederico, Phys. Rev. A66, 043619 (2002) and references therein.

[15] E. H. Lieb, R. Seiringer, and J. Yngvason, Phys. Rev. A61, 043602 (2000).

[16] See e.g. P. Ring and P. Schuck, The Nuclear Many-Body Problem, Springer Verlag 1980, section 5.6.

[17] F. Dalfovo, L. Pitaevski, and S. Stringari, Phys. Rev. A54, 4213 (1996).

[18] J. P. Burke, C. H. Greene, and J. L. Bohn, Phys. Rev. Lett. 81, 3355 (1998).

[19] M. Koashi and M. Ueda, Phys. Rev. Lett. 84, 1066 (2000); M. Ueda and M. Koashi, Phys. Rev. A65, 063602 (2002).

[20] C. K. Law, H. Pu, and N. P. Bigelow, Phys. Rev. Lett. 81, 5257 (1998); H. Pu, C. K. Law, S. Raghavan, J. H. Eberly, and N. P. Bigelow, Phys. Rev. A60, 1463 (1999).

[21] Tin-Lun Ho and Sung Kit Yip, Phys. Rev. Lett. 84, 4031 (2000).

[22] E. H. Lieb and R. Seiringer, Phys. Rev. Lett. 88, 170409 (2002).

[23] E. H. Lieb, R. Seiringer, J. P. Solovej, and J. Yngvason, arXiv:math-ph/0204027 (11 Apr 2002).

[24] H. Feshbach, Ann. Phys. (N.Y.) 19, 287 (1962).

[25] M. W. Zwierlein, C. A. Stan, C. H. Schunck, S. M. F. Raupach, S. Gupta, Z. Hadzibabic, and W. Ketterle, arXiv:condmat/0311617v1 (27 Nov 2003)

[26] E. Timmermans, P. Tommasini, M. Hussein, and A. K. Kerman, Phys. Reports 315, 199 (1999). 
[27] Ph. Courteille, R. S. Freeland, D. J. Heinzen, F. A. van Abeelen, and B. J. Verhaar, Phys. Rev. Lett. 81, 69 (1998).

[28] C. Chin, V. Vuletić, A. J. Kerman, and S. Chu, Phys. Rev. Lett. 85, 2717 (2000).

[29] P. J. Leo, C. J. Williams, and P. S. Julienne, Phys. Rev. Lett. 85, 2721 (2000).

[30] K. Gral, T. Khler, S. A. Gardiner, E. Tiesinga, and P. S. Julienne, arXiv:cond-mat/0312178 v2 (15 Dec 2003)

[31] A. N. Salgueiro, M. C. Nemes, M. D. Sampaio, and A. F. R. de Toledo Piza, Physica A290, 4 (2001).

[32] P. Tommasini, E. J. V. de Passos, A. F. R. de Toledo Piza, M. S. Hussein, and E. Timmermans, Phys. Rev. A67, 023606 (2003).

[33] V. A. Yurovsky, A. Ben-Reuven, P. S. Julienne, and C. J. Williams, Phys. Rev. A60, R765 (1999).

[34] E. Donley, N. R. Claussen, S. T. Thompson, and C. E. Wieman, Nature 417, 529 (2002).

[35] C. A. Regal, C. Ticknor, J. L. Bohn, and D. S. Jin, Nature 424, 47 (2003).

[36] M. Greiner, C. A. Regal, and D. D. Jin, arXiv:condmatt/031172 v1 (7 Nov 2003).

[37] M. W. Zwierlein, C. A. Stan, C. H. Schunck, S. M. F. Raupach, S. Gupta, Z. Hadzibabic, and W. Ketterle, arXiv:condmatt/0311617 v1 (27 Nov 2003).

[38] S. J. J. M. F. Kokkelmans, G. V. Shliapnikov, and C. Salomon, arXiv:cond-mat/0308384 v3 (15 Jan 2004); D. S. Petrov, C. Salomon and G. V. Shliapnikov, arXiv:cond-mat/0309010 v1 (30 Aug 2003); M Wouters, J. Tempere, and J. T. Devrese, arXiv:cond-mat/0312227 (9 Dec 2003).

[39] R. Wynar, R. S. Freeland, D. J. Han, C, Ryu, and D. J. Heizen, Science 287, 1016 (2000); C. J. Williams and P. S. Julienne, ibid. p. 986.

[40] M. R. Matthews, D. S. Hall, D. S. Jin, J. R. Ensher, C. E. Wieman, E. A. Cornell, F. Dalfovo, C. Miniti, and S. Stringari, Phys. Rev. Lett. 81, 243 (1998); D. S. Hall, M. R. Matthews, J. R. Ensher, C. E. Wieman, and E. A. Cornell, Phys. Rev. Lett. 81, 1539 (1998).

[41] J. Stenger, S. Inouye, D. M. Stamper-Kurn, H.-J. Miesner, A. P. Chikkatur, and W. Ketterle, Nature 396 (1998).

[42] N. Bogoliubov, J. Phys. USSR, 11, 231947.

[43] L. D. Landau and E. M. Lifshitz, Statistical Physics, Pergamon Press, London-Paris (1959), §76.

[44] T. D. Lee, K. Huang, and C. N. Yang, Phys. Rev. 106, 1135 (1957).
[45] K. Huang and C. N. Yang, Phys. Rev. 105, 767 (1957).

[46] T. D. Lee and C. N. Yang, Phys. Rev. 105, 1119 (1957).

[47] See A. Griffin, Phys. Rev. B53, 9341 (1996) and references therein.

[48] P. Tommasini, E. J. V. de Passos, M. O. C. Pires, and A. F. R. de Toledo Piza, Beyond HFB Approximation: One and two quasiparticle excitations, submitted (2003).

[49] V. N. Popov, Functional Integrals and collective modes, Cambridge University Press, New York, (1987), Chap. 6.

[50] P. Ring and P. Schuck, The Nuclear Many-Body Problem, Springer Verlag (1980)

[51] M. R. Andrews, C. G. Townsend, H.-J. Miesner, D. S. Durfee, D. M. Kurn, and W. Ketterle, Science 275, 637 (1997).

[52] H. Wallis and H. Steck, Europhysics Letters 41, 477 (1998).

[53] J. Javanainen and M. Wilkens, Phys. Rev. Lett. 78, 4675 (1997).

[54] J. Javanainen and S. M. Yoo, Phys. Rev. Lett. 76, 161 (1996). See also ref. [53]!

[55] Y. Shin, M. Saba, T. A. Pasquini, W. Ketterle, D. E. Pritchard and A. E. Leanhardt, arXiv:cond-mat/0306305 v2 (17 Jul 2003)

[56] B. P. Anderson e M. A. Kasevich, Science 282, 1686 (1998).

[57] F. S. Cataliotti, S. Burger, C. Fort, P. Maddaloni, F. Minardi, A. Trombettoni, A. Smerzi, and M. Inguscio, Science 293, 843 (2001); P. Pedri, L. Pitaevskii, S, Stringari, C. Fort, S. Burger, F. S. Cataliotti, P. Maddaloni, F. Minardi, and M. Inguscio, Phys. Rev. Lett. 87220401 (2001).

[58] M. Greiner, O. Mandel, T. Esslinger, T. W. Hõnsch, and I. Bloch, Nature 415, 39 (2002).

[59] If you need a primer on Wanier functions, try F. B. Pedersen, G. T. Einevoll and P. C. Hemmer, Phys. Rev. B44, 5470 (1991).

[60] J. Schwinger, Quantum Theory of Angular Momentum, L. Biedenharn and H. Van Dam, Eds., Academic Press, New York (1965)

[61] J. L. van Hemmen and A.St, Theory of mesoscopic quantum tunneling in magnetism: a WKB approach in Quantum Tunneling of Magnetization - OTM '94, L. Gunther and B. Barbara, Eds., Kluwer Academic Publishers Dordrecht/Boston/London 1995.

[62] D. Galetti and A. F. R. de Toledo Piza, Physica A214, 207 (1995).

[63] Gh.-S. Paraoanu, Phys. Rev. A67, 023607 (2003). 